www.anatomy.org.tr

doi:10.2399/ana.20.001s

\title{
Abstracts of the 21st National Anatomy Congress 27-29 November 2020, Online, Turkey
}

\section{Invited Lectures}

\author{
(I-1 - I-5)
}

\section{1-1}

An unforgettable legend: Prof. Dr. Sami Zan

Güler K

Department of Internal Medicine, Istanbul Faculty of Medicine, Istanbul University, Istanbul, Turkey

"The risorius muscles of a physician should always work" was only one of many invaluable sayings I could remember of this passionate, inspiring, and suave gentleman with a cunning glance behind his glasses. Sami ZAN was born in 1921 in Istanbul. He graduated from the Haydarpaşa High School in 1940 and subsequently started his medical training at Istanbul Faculty of Medicine. Prior to his internship, he transferred to the newly founded Faculty of Medicine at Ankara University and graduated in 1946. Following his military service, he completed his mandatory health service in the İdil province of Mardin. He returned to the Istanbul Faculty of Medicine in 1948 as a research assistant in the Institute of Anatomy. He was appointed as an associate professor in 1955, a full professor in 1966, and as the Head of Department of Anatomy in 1978 until his passing due to a heart attack in 1984. His expertise in anatomical knowledge and educational skills identified him as a "Master Teacher" and resulted in superb lectures that were best described as theatrical performances. As a prominent lecturer he simplified most complex anatomical concepts by integrating low fidelity models including cardboards, hoses, kettles, mess kits, sheaths, water filled balloons or even food such as eggs and sandwiches. For example, in his lectures on the prostate he always used a tangerine that symbolized benign prostate hyperplasia. He extracted the fruit without damaging the skin and emphasized the current surgical approach to the disease. He also used chalk to prepare hand drawn figures and diagrams in advance of his lectures. Nevertheless, his most prominent feature was probably his excellent talent for interacting with students and being approachable to all of them. To achieve this, he initiated the "Philosophical Anatomy" talks before his lectures. These pre-lecture talks were conducted in a conversational style and covered the lecture topic of the day involving a general knowledge regarding anatomy, physiology, and clinical practice integrated with interesting information that he collected from news or magazine articles. The Philosophical Anatomy lectures were so popular that students from various faculties such as law, dentistry, or pharmacy, and even common folk attended them occasionally. Every time a generation of physicians as his pupils reflect on his jokes and advises, they relive his lectures and aspire to share them in order to keep them alive.

Keywords: anatomy education, Sami Zan

\section{I-2}

Arthroscopic anatomy and pathologies of the shoulder Özkan MH

Department of Orthopedics and Traumatology, Faculty of Medicine, Dokuz Eylül University, Izmir, Turkey

In order to understand musculoskeletal pathologies of the shoulder region, comprehending the physioanatomy of the glenohumeral, acromioclavicular, and scapulothoracic joints along with the muscles responsible for the synchronization of 
these structures is essential. Additionally, emphasizing the muscular, skeletal, and neural structures of the region with a functional perspective is vital for performing safe and adequate open surgical or arthroscopic procedures. This presentation will outline the surgical anatomy of the shoulder by evaluating the normal and variational structures from superficial to deep with a macroscopic and arthroscopic point of view. It is important to pay attention to patient positioning and technical details for achieving a wholesome diagnostic arthroscopy. Therefore, normal muscular and neurovascular structures and their relations with arthroscopic portals will be examined before outlying intraarticular structures. Following the portal access based on anatomical landmarks, a systematic evaluation of intraarticular and subacromial structures will be performed with the help of a 30 degree arthroscope.

Keywords: arthroscopy, shoulder anatomy

\section{I-3}

\section{Do we need to extend Terminologia Anatomica and Terminologia Neuroanatomica?}

Kachlík D

Department of Anatomy, Second Faculty of Medicine, Charles University, Prague, Czech Republic

\section{I-4}

A new animal model of causing proteinopathic damage in motor neurons: does it also show promise in delaying aging?

Ulupinar $\mathrm{E}^{1,2}$

'Department of Anatomy, Faculty of Medicine, Eskişehir Osmangazi University, Eskişehir, Turkey; ${ }^{2}$ Department of Interdisciplinary Neuroscience, Institute of Health Sciences, Eskişehir Osmangazi University, Eskişehir, Turkey

Nucleus is an organelle that plays a critical role in the regulation of gene expression in the cell. Especially in postmitotic cells like neurons, it is necessary to maintain the transport functions between the nucleus and the cytoplasm throughout the life in order to maintain the genomic integrity and to operate DNA repair mechanisms. In recent studies, it has been pointed out that nucleocytoplasmic transport defects are a common component of both physiological aging and neurodegenerative diseases characterized by toxic protein aggregates. Some patients with Amyotrophic lateral sclerosis (ALS), a disease characterized by the progressive loss of motor neurons, develop behavioral and cognitive losses and early dementia due to atrophy in the frontotemporal cortex. In the literature, it has been shown that DNA/RNA binding proteins such as TDP-43 and FUS, which should normally be found in the nucleus of neurons and glial cells, form pathological aggregates in the cytoplasm of neurons in the post-mortem brain specimens of such patients. In this study, a novel animal model was devel- oped by using viral mediated gene transfer method to alter the genes encoding TDP-43 protein specifically in the cortical motor neurons of young adult rats. Following systemic injection of viral vectors, changes in locomotor activities of rats were evaluated with different behavioral tests during 6 weekperiod and neuronal densities in the motor cortex were calculated by morphometric methods. In confocal and electron microscopic analyzes, performed to investigate ultrastructural changes causing a significant reduction in the corticospinal motor neurons, serious mitochondrial damage was observed in neurons, as well as defects in the nuclear membrane and endoplasmic reticulum. These results show that many aspects of structural and functional defects commonly referred to as "TDP-43 pathology" in humans, might be imitated in animal models. The development of such animal models is important in terms of elucidating the common mechanisms observed in both physiological and pathological conditions, and also in future studies discovering new therapeutic and /or preventive approaches. This work was supported by the TÜBİTAK-1001 project numbered $116 \mathrm{~S} 408$.

Keywords: TDP-43, proteinopathy, viral vector, motor neuron, aging, morphometry

\section{$1-5$}

Anatomy in the context of translational medicine research: from basic sciences to community

Durgun B

Department of Anatomy, Faculty of Medicine, Çukurova University, Adana, Turkey

Objective: This study aims to review the definition and the explanation of translational medicine research; the difference between applied and translational research and how the anatomical studies are designed in the context of translational medicine.

Methods: By adding the author's own experience, this study is a literature review made with the idea that the anatomical studies should not only be limited to basic research but they should also lead the way for translational research.

Results: Translational research involves interdisciplinary integration among basic, clinical, practice, population, and policybased research. The main goal of translational research is to get faster scientific discovery into community benefit. Unlike applied sciences, translational research is specifically designed to improve health outcomes. Translational medicine, on the other hand, uses advanced basic research methods such as cell cultures, genetic studies or animal models to develop new treatments, drug or medical procedures. Translational Medicine means different things for different people. For many, the term refers to the application of basic science discoveries to meaningful improvement in patient outcome (T1 research). In this approach, the endpoint is the production of a 
new treatment that can be used clinically or commercialized. For others, e.g. for health services researchers and public health investigators whose studies focus on health care and health as the primary outcome, translational medicine research refers to translating research into practice, e.g. knowledge actually reaches the patients or populations (T2 research). T2 research aims to improve quality by improving access, reorganizing and coordinating systems of care, helping clinicians and patients to change behaviours and strengthening the patientclinician relationship. The production of a new drug, an endpoint for "bench-to-bedside" translational research, is only the starting point for the second approach. Anatomy should be in translational medicine research. The bench is where discovery begins, should not only in a laboratory surrounded by test tubes and pipettes, but rather, can happen in the anatomy laboratory through dissection. The "bench-to-bedside" model can be applied in clinical anatomy by answering clinical problems with anatomic explanations and translating the discoveries to treatments in patients.

Conclusion: In the light of new technologies and approaches to scientific research, anatomy studies can be designed in the context of translational medicine. It's just the time shifting the lens of anatomy to translational research. Thus, the translation of anatomic research to improved clinical outcomes lead the new way for future developments.

Keywords: anatomy research, bench-to-bedside, translational medicine, translational research 


\section{Panels}

(PL-1 - PL-14)

\section{Clinical Anatomy Panel 1}

\section{Surgical anatomical studies in Ege University Faculty of Medicine}

Makay $\ddot{O}^{1}$, Yeniel AÖ ${ }^{2}$, Uyanıkgil $\mathrm{Y}^{3}$, Çelik S ${ }^{4}$, Bilge $\mathrm{O}^{4}$

'Department of General Surgery, Faculty of Medicine, Ege University, Izmir, Turkey; ' Department of Obstetrics and Gynecology, Faculty of Medicine, Ege University, Izmir, Turkey; ' ${ }^{3}$ epartment of Histology and Embryology, Faculty of Medicine, Ege University, Izmir, Turkey; ${ }^{4}$ Department of Anatomy, Faculty of Medicine, Ege University, Izmir, Turkey

Objective: With the cooperation of surgical sciences and basic sciences, it is aimed to develop educational models, to conduct research, to produce projects and publications. In this direction, surgeons, anatomists and histologists have collaborated in Ege University Faculty of Medicine.

Methods: Basic scientists have participated in surgical operations in the clinics, and clinicians have participated in practice in basic sciences laboratories. Anatomists have participated in scarless thyroid surgery operations and examined the surgical stages. Subsequently, surgeons performed the operation on light fixation cadavers. The stages of the surgery in cadavers with light fixation were determined, the training model was designed and application courses were held. Training models were planned in a similar way with gynaecologists and 5 pelvic surgery courses were conducted. With the experience gained, it is planned to develop a new surgical technique and device. Measurements were made for devices and prototypes were tested on female cadavers. Applications in surgeries were examined histologically as well as macroscopically. Surgeons, anatomists and histologists took part in the prevention of surgical complications and device development by interpreting the examination of surgical structures together. Clinical and surgical collaborations were developed by the participation of residents and experts in each of the stages.

Results: Surgical anatomical projects, course models and publications have been produced. Course models developed for thyroid surgery were made for the first time in our country and were performed 4 times ( 3 international, 1 national in-house) with international participation. Surgery training in the courses have been provided and new operative scarless technique has become widespread in our country. The first atlas was made for scarless thyroid surgery, and publications were produced from patient and cadaver studies. In the field of gynaecological pelvic surgery, the KOSGEB project was carried out and 5 (3 international, 2 national) pelvic floor surgery courses were held. The first European Union Horizon 2020 projects were taken in the field of medical devices in our country, and new surgical techniques and devices were realized.

Conclusion: The cooperation of basic sciences and surgical sciences enables the development of new educational models,

scientific publications and projects in the national and international field. These collaborations should be maintained and expanded steadily. Training curricula of young anatomists and surgeons on this subject should be arranged.

Keywords: surgical anatomy, education, research, innovation, surgical anatomy projects

\section{PL-1}

The journey of transoral endoscopic thyroidectomy with the cooperation of surgery and anatomy

Makay Ö

Department of General Surgery, Faculty of Medicine, Ege University, Izmir, Turkey

\section{PL-2}

From cadaver to European Union Project: developing the surgery and equipment

Yeniel AÖ

Department of Obstetrics and Gynecology, Faculty of Medicine, Ege University, Izmir, Turkey

\section{PL-3}

Histology and Embryology and Anatomy with a clinical perspective: morphology once more again

Uyanıkgil Y

Department of Histology and Embryology, Faculty of Medicine, Ege University, Izmir, Turkey

\section{PL-4}

The "from basic to clinic, from clinic to basic" approach to surgical anatomy

\section{Çelik S}

Department of Anatomy, Faculty of Medicine, Ege University, Izmir, Turkey

Clinical Anatomy Panel 2 Clinical anatomy from past to present

\section{PL-5}

Prof. Dr. İbrahim Veli Odar and clinical anatomy

Tekdemir İ

Department of Anatomy, School of Medicine, Ankara University, Ankara, Turkey 


\section{PL-6}

Colorectal surgical anatomy

Kuzu MA

Department of General Surgery, School of Medicine, Ankara University, Ankara, Turkey

\section{PL-7}

Surgical anatomy of the knee from an anatomist's perspective

Açar Hİ

Department of Anatomy, School of Medicine, Ankara University, Ankara, Turkey

\section{PL-8}

Surgical neuroanatomy

Kahiloğulları G

Department of Neurosurgery, School of Medicine, Ankara University, Ankara, Turkey

\section{Anatomy Education Panel \\ Methods used to increase learning in online anatomy education}

\section{PL-9}

An example for reinforcing anatomy laboratories with a modified online flipped-classroom education

\section{Akkın SM}

Department of Anatomy, Faculty of Medicine, SANKO University, Gaziantep, Turkey

\section{PL-10}

General problems and practice in distance anatomy education

Ünver Doğan N

Department of Anatomy, Faculty of Medicine, Selçuk University, Konya, Turkey

\section{PL-11}

Anatomy and social media: what, how much, and to who?

Tunali $\mathrm{S}$

Department of Anatomy, Faculty of Medicine, TOBB University of Economics and Technology, Ankara, Turkey

\section{PL-12}

Anatomy lessons on YouTube, how much wrong is there?

Albay S

Department of Anatomy, Faculty of Medicine, Süleyman Demirel University, Isparta, Turkey
Due to the situation which the world is currently in, medical education is also carried out online. The issue that concerns us as anatomist is that our laboratory lesson can be done via distance education. In this case, in order to ensure that the level of our education is as well as possible, our lectures on cadaver and models can be conducted in front of a camera via the system our university provided us. However, it was seen that this approach leads to some deficiencies such as fluency and intelligibility. That's why, recording laboratory lectures online and uploading them to YouTube, a website that can easily be accessed by students was found more appropriate by our department. It is also known that there are hundreds of videos on the mentioned website currently available. When these videos are analyzed, a great deal of the lecturers in videos were found out to be incapable of giving anatomy lessons and full of terminology and information errors. The fact that this information pollution and videos full of errors lead our students to learn the lecture inaccurately was clearly observed. In order to prevent these mistakes being made by us, video lectures prepared by us were shared with students after a detailed examination by at least three of our instructors. Laboratory lessons are the sine qua non of anatomy learning, meaning in theoretical lectures, unity cannot be provided without three-dimensional vision, seeing it from different angles and touching. Even though it is impossible to provide this via distance learning, we attempted to minimize this deficiency through our video laboratory lectures even though it is two-dimensional.

Keywords: distance education, anatomy laboratory, YouTube

\section{PL-13}

Case-based gross anatomy learning for abdominal cavity using MR imaging of cadavers and delivered utilizing extended reality: web technology

Nakamatsu NA ${ }^{1}$, Torigoe $\mathrm{T}^{1}$, Mikami B ${ }^{1}$, Thompson $\mathrm{JD}^{1,2}$, Rettenmeier $\mathrm{C}^{3}$, Lozanoff BK ${ }^{1}$, Kaya $\mathrm{B}^{1}$, Smith $\mathrm{A}^{1}$, Lee $\mathrm{U}^{1,4}$, Aytaç $\mathrm{G}^{5}$, Lozanoff $\mathrm{S}^{1,2}$

'Department of Anatomy, Biochemistry \& Physiology, John A. Burns School of Medicine, Hawaii University, Hawaii, USA; 'Radial3D Inc., Hawaii, USA; ${ }^{3}$ UHIQMC MRI Research Center, John A. Burns School of Medicine, Hawaii University, Hawaii, USA; ' $D$ epartment of Anatomy, College of Medicine, The Catholic University of Korea, Seoul, Korea; ${ }^{5}$ Department of Anatomy, Faculty of Medicine, Yüksek Ihtisas University, Ankara, Turkey

Objective: Anatomy remains an essential element of medical education. In addition to electronic delivery of course content, the use of extended reality (XR) has been shown to complement traditional learning techniques in anatomy. Knowledge of complex spatial relationships of the organs is necessary for complete understanding of imaging studies, diagnosis, surgical approaches, and treatment plans. The purpose of this study was to develop and apply a case-based approach in gross anatomy, utilizing XR technology, to promote student exploration and engagement.

Methods: Learning modules were prepared for the study. The learning modules were tested in 20 volunteer medical students 
from different cities in Turkey by creating virtual classrooms with a program offered by distance education. The learning modules were comprised of MRI scanning of cadavers, radiological pathological and anatomical assessment, as well as case development based on XR visualizations. Case information (Subject, History and Physical Exam) was presented to students who generated and recorded hypotheses during the online class. Students subsequently reviewed Radiology and Pathology reports and accessed relevant XR visualizations. After the online, class students were asked to fill in a form questioning their experiences related to the lesson. Answers were on a Likert scale (poor-excellent, 0-5 or not likely-very likely 0-5).

Results: Some of the critical questions in the study, the percentages of the answers to these questions, and (Likert scores) were as follows: Was viewing the MR scans of the cadavers useful as a tool for online learning? 90.9\% (5), 9.1 (4); Did viewing the MR scans of the cadavers provide an understanding of the relevant anatomy? 100\% (5); Did the additional 3D models on Sketchfab provide a better understanding of the anatomical relationships? $100 \%$ (5); Would you like to see more online interactive sessions using the MR scans of cadavers? $100 \%$ (5); Would you use similar resources in the future? 91.7\% (5).

Conclusion: Data from this study suggests that the online delivery and learning module were well received by medical students. Given the state of the current global pandemic, students are being forced to learn remotely to ensure the safety of students and faculty and to reduce the risk of spread of COVID-19. Besides all the other aspects, this methodology would ensure students contactless learning experiences that enhance the understanding of difficult pathologic conditions even though students are unable to physically work with cadavers.

Keywords: anatomy, medical education, XR technology, distance learning

\section{PL-14}

Experience sharing about online assignments and practical lessons in Cerrahpaşa Medical Faculty Department of Anatomy during Covid-19 pandemic

Doğancı ÖI, Taștan ÖA

Department of Anatomy, Cerrahpaşa Medical Faculty, Istanbul UniversityCerrahpaşa, Istanbul, Turkey
The Covid-19 pandemic influenced the entire educational order and forced academicians to find new solutions. We, as Cerrahpasa Medical Faculty Department of Anatomy, wanted to share the solutions and methods we have implemented in order to perform anatomy practices in this process. Anatomy lectures and practical lessons are carried out by online distance education methods during this pandemic. When planning the online anatomy practices for 1st, 2nd, and 3rd year Medical Faculty students in both Turkish and English programs, we put forward some ideas about how we can create the closest simulation resembling the learning process at the times before pandemic where students spend time in the laboratory. Based on the ideas of actively including the student in the learning process in practical lessons, asking them questions to find their own answers, and giving them time to investigate, we have prepared assignments for each practical lesson according to the subject distribution compatible with the theoretical lectures in related courses. We have assigned our assignments to students as forms within the Google Classroom application. In each practical lesson, an assignment was published, and the answers to the previous assignment were discussed in an online lecture in a Q\&A format via Zoom application. The duration of the assignments given was determined as the time between two consecutive practical lessons and requested to be delivered by the students prior to the practical lesson related to the assignment. Feedbacks about the practice are taken from students after courses. There was a common opinion that the assignments motivated the students to study in groups via computers or smartphones, that they spend long hours at home doing these assignments, and that their study time was longer. This practice required a longer preparation period for assignments compared to the times of face-to face practical lessons, which in turn increased the workload for us educators. The idea of an online assignment system we applied being integrated into normal anatomy practices and the possibility of more efficient practical lessons at the laboratory using cadavers with the students who had researched the structure beforehand and coming to the laboratory with prior knowledge, has become prominent.

Keywords: covid-19 pandemic, anatomy education, student integration 


\section{Oral Presentations \\ (0-01 - 0-94)}

\section{0-01}

Investigation of students' attitudes towards applied distance education in the Covid-19 pandemic process in higher education institutions: example of Meram Faculty of Medicine

Yılmaz MT, Saygın DA, Kabakcı ADA

Department of Anatomy, Faculty of Medicine Meram, Necmettin Erbakan University, Konya, Turkey

Objective: In the Covid-19 pandemic process of this study, the Necmettin Erbakan University, Meram Faculty of Medicine aimed to investigate students' attitudes towards distance education.

Methods: 310 (197 females 63.55\%, 113 males 36.45\%) students studying in the Necmettin Erbakan University, Meram Faculty of Medicine were included in the study. The data was obtained with the questionnaire form created using the 21-item "Attitude Towards Distance Education Scale". In the study, demographic data on gender and classes were obtained. The data given in the hand were loaded into the SPSS 20.00 program and the answers given to the questionnaire were evaluated in terms of gender and class. $\mathrm{p}<0.05$ value was considered significant.

Results: While $75.17 \%$ of the students access education by using a computer, $3.22 \%$ tablet and $21.61 \%$ by phone, it has been observed that both genders prefer the computer. There was no significant difference in attitude towards distance education by gender and classes $(p>0.05)$. When the efficiency of the lessons was questioned with the distance education method during the pandemic process, 39.68\% (123) of the students gave the answer as efficient for the lessons and 60.32\% (187) inefficient. After the pandemic process was completed, the education system that the students would prefer when the conditions were suitable were asked and $78.06 \%$ (242) of them stated that they would like to continue their education with formal education, and $21.94 \%$ (68) of them stated their preferences as continuing distance education. However, they stated results in favor of distance education in questions such as "Access to information is fast due to the sharing of knowledge in distance education on the internet", "I think that there is no time and place limitation in distance education", "I think that it provides the flexibility to repeat distance education as desired".

Conclusion: According to the results, the positive and negative aspects of distance education at student level were tried to be revealed as clearly as possible. Considering the results obtained, it is seen that 1 st and 2 nd term students of the Faculty of Medicine benefit from the advantages of distance education, but their education preferences are in favor of formal education, no matter how technology age young people are.

Keywords: covid 19, distance education, medicine faculty, student, attitude

\section{0-02}

Can flipped classroom strategy meet the needs of students regarding anatomy education?

Cüneyit $\dot{I}^{1}$, Kara $\mathrm{CO}^{2}$, Süral S , Adıgüzel E ${ }^{1}$

'Department of Anatomy, Faculty of Medicine, Pamukkale University, Denizli, Turkey; '2Ear, Nose and Throat Department, Faculty of Medicine, Pamukkale University, Denizli, Turkey; ${ }^{3}$ Department of Educational Sciences/Department of Education Programs and Instruction, Faculty of Education, Pamukkale University, Denizli, Turkey

Objective: With the changing generation of students, learning and teaching strategies in medical education also change. Flipped classroom model (FC) is the most easily implemented student-centered model of these strategies. Although FC is frequently on agenda due to pandemic, literature shows limited experience on this topic in medical education in our country. Our aim in this study is to evaluate whether problems observed in medical student feedback on anatomy education could be solved with FC. In this paper, evaluation of feedback of 6th grade students who took lessons with FC and feedback of 3rd grade students who received anatomy education and preparatory findings of study on application of this model to anatomy lessons are presented.

Methods: FC consists of contents prepared by instructor and transfer of theoretical education given in classroom outside the classroom. Students study subjects at home before they come to class, so focus is on student-centered active learning during time spent in the classroom. In order to prepare scale for model, feedback on ENT internship, which was applied to FC, were evaluated. Student feedback on anatomy education was received from 3rd grade students who had received anatomy training one year ago. Feedbacks were evaluated with descriptive analysis by two independent evaluators, and were discussed together and similar feedback were grouped under the same headings. Attitude scale was created by using answers to openended questions. It was planned to apply these scales to 2 nd grade students before and after FC. This research was approved by Pamukkale University's non-interventional clinical research ethics committee (60116787-020/50334).

Results: It was determined that students' feedback on anatomy education emphasized shortage of theoretical and practical 
hours, and they wanted to increase both training hours. It was understood that students wanted presentations to be shared before classes and practice hours. In feedback about FC, they stated that they could spare time for patients in the clinic because they had studied lessons before coming to clinic.

Conclusion: As can be understood from student feedback, time spent in didactic lessons in anatomy education can be more useful with FC. In classroom lessons, students will be able to reach higher learning levels at anatomy knowledge level by reinforcing information they have learned with video lessons with interactive methods such as question and answer, case-problem solutions. In addition, if theoretical lessons are shared with students as pre-class videos, more time can be allocated to practice hours. Flipped classroom strategy can be a solution to students' wishes.

Keywords: anatomy, education, flipped classroom, student

\section{0-03}

Students' opinions about distance anatomy education and the unique features to distance anatomy education: Covid-19 example

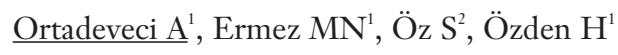

'Department of Anatomy, Faculty of Medicine, Eskişehir Osmangazi University, Eskişehir, Turkey; 'Health Services Vocational School, Eskişehir Osmangazi University, Eskişehir, Turkey

Objective: Especially in the last 25 years, technology has begun to dominate all areas of our lives. Education is one of the areas where it is impossible to be independent from technology. Many changes that occur in terms of method, material and application in education have become commonplace. Distance education has made a very sudden and holistic entry into the education field due to the COVID-19 pandemic. Although it is relatively easy to find a suitable alternative for theoretical training, the efficiency of distance education in areas that require practice such as medical education is still controversial. Traditional education of Anatomy is based on learning by seeing and touching with cadavers or plastic materials in a way that enables three-dimensional thinking. Due to the lack of face-to-face laboratories in the distance learning process, the students were deprived of the traditional anatomy learning style. Our aim in this study is to evaluate students' views on the efficiency of distance anatomy education and to increase the efficiency of remote anatomy education in the future.

Methods: Our questionnaire that we used in the study was applied to 1st grade students of Eskişehir Osmangazi University Faculty of Medicine in the 2019-2020 academic year. A total of 239 people out of 301 people completed the survey. In the introduction part of our survey, there are expressions explaining the survey and its aims and asking for voluntary consent. In the next section, there are demographic questions that collect data such as age, gender, financial income of the family, and location during education. In the third part, there are suggestions about distance anatomy education. In the last part, there are suggestions about the comparison of distance Anatomy education with other basic science courses. The last question of the questionnaire was questioning recommendations for a better distance anatomy education. The survey study was prepared on Google forms and sent to students via the web.

Results: $66.1 \%$ of our students marked "I do not agree at all" and $16.3 \%$ of them marked "I agree less" with the statement of "Distance anatomy education is more efficient than traditional anatomy education". On the other hand, $47.3 \%$ of the students stated that they did not agree at all with the statement of "Distance anatomy education is suitable for me". The total percentage of students who did not agree at all or agreed less with the statement "I was satisfied with the theoretical education in distance anatomy education" was $58.6 \%$, when it comes to practical education the percentage was $79.9 \%$. In the openended question at the end of the questionnaire, many students mentioned the difficulty of distance anatomy education and expressed their desire to make the practical lessons face-toface.

Conclusion: According to the responses of 239 ESOGU Faculty of Medicine 1st grade students who participated in our survey, the efficiency of distance anatomy education, especially in terms of practice lessons, was found to be lower than traditional anatomy education.

Keywords: distance education, traditional education, medical education, anatomy education, medical students

\section{0-04}

The effect of Covid-19 process on anatomy education at the faculty of medicine

Karaca Bozdağ $Z^{1}$, Candır BN ${ }^{1}$, Gayretli $\mathrm{O}^{2}$, Arı $Z^{1}$

${ }^{\prime}$ Department of Anatomy, Faculty of Medicine, Istanbul Yeni Yüzyıl University, Istanbul, Turkey; ${ }^{2}$ Department of Anatomy, Istanbul Faculty of Medicine, Istanbul University, Istanbul, Turkey

Objective: Due to the coronavirus (COVID-19) epidemic that develops due to severe acute respiratory syndrome coronavirus 2 (SARS-CoV-2) infection, an online education process has been started at universities. We aimed to investigate the effects of this process on anatomy education in our university, Faculty of Medicine.

Methods: The questionnaire form prepared over the Google Forms application for the 1st and 2nd classes of Faculty of Medicine students who actively take anatomy lessons in the spring semester of the 2019-2020 academic year of Istanbul Yeni Yüzyll University, after the approval of the ethics committee (date: 09.07.2020, number: 2020/07-486) ) was applied on a voluntary basis. The form consisted of 9 questions for general information, 5 questions for 1 st class students, 7 ques- 
tions for 2 nd class students, and 4 questions for comparing the anatomy with other medical faculty lessons. For statistical analysis SPSS 22 (SPSS IBM, Turkey) program was used and $\mathrm{p}<0.05$ was considered significant.

Results: One hundred and eighty students participated in the study, 97 of which were 1 st class students and 83 were 2 nd. It was observed that students who use the internet for 1-2 hours per day do not find online education appropriate compared to students who have 3-4 hours or more $(\mathrm{p}=0.046)$. Compared to the students who answered yes to the question "I like anatomy lesson", those who answered no; it was found that their satisfaction with the teaching of the anatomy lesson before and after the pandemic was higher $(\mathrm{p}=0.003)$. Compared to theoretical lessons, the proportion of students who argued that practice lessons were negatively affected was higher $(\mathrm{p}=0.002)$. However, no statistically significant difference was found between performing anatomy practice lessons via dissection or modeling $(\mathrm{p}=0.438)$. In addition, it was found that both anatomy theoretical $(\mathrm{p}=0.014)$ and practical training $(\mathrm{p}<0.001)$, which were taken face to face before the Covid-19 pandemic, were more effective than the theoretical and practical training of other medical faculty courses.

Conclusion: Our study revealed that the Covid-19 process negatively affected anatomy education, and that students benefit more from face-to-face education. The high dissatisfaction rate of students in practical training is proof that anatomy is a science based on visual learning.

Keywords: covid-19 pandemic, anatomy education, online education, anatomy training in pandemic, anatomy practice training

\section{0-05}

Evaluation of anatomy exams conducted by distance education in medical and dentistry faculties during the pandemic period with student feedback

$\underline{\text { Solmaz E }}{ }^{1}$, Doğan $M E^{2}$, Fazlığulları $Z^{1}$, Sevindik B ${ }^{1}$, Saylam $\mathrm{H}^{1}$, Ünver Doğan $\mathrm{N}^{1}$

'Department of Anatomy, Faculty of Medicine, Selçuk University, Konya, Turkey; ${ }^{2}$ Department of Public Health, Faculty of Medicine, Selçuk University, Konya, Turkey

Objective: This study was carried out to evaluate the questionnaire made to the Faculty of Medicine and Faculty of Dentistry students to measure the anatomy theoretical and practical exams in distance education during the pandemic process in the spring term in the 2019-2020 academic year.

Methods: This descriptive study was conducted with 1st and 2nd-year students of Selçuk University Faculty of Medicine and Faculty of Dentistry. Questionnaire questions were prepared in a Google document to be answered with a 5-point Likert scale and the questionnaire access link was sent to 793 students by phone message. Data was evaluated with the SPSS
22.0 package program. The average and percentage distributions of the scores of the participants were calculated.

Results: 152 1st-year, 103 2nd-year students from Faculty of Medicine, 38 1st-year, 102 2nd-year students from Faculty of Dentistry, a total of 395 students participated in the study. The satisfaction rate was determined as $80 \%$ for the Faculty of Medicine students and $74 \%$ for the Faculty of Dentistry students. Students' opinions, criticisms, and requests about the exams were evaluated.

Conclusion: Regulations are important for improvement and development in the measurement and evaluation of anatomy lectures in distance education. The inclusion of the students in the process with their feedback is valuable in terms of eliminating the existing imperfections and having better quality and efficient period.

Keywords: pandemic, distance education, anatomy exam, questionnaire, measurement and evaluation, feedback

\section{0-06}

The relationship between hand A1 flexor pulley and digital palmar crease

Vural A ${ }^{1}$, Kaptan AY ${ }^{2}$, Kanatlı $\mathrm{U}^{3}$, İnce $\mathrm{MS}^{4}$, Aytaç $\mathrm{G}^{5}, \underline{\text { Gözil R}}{ }^{5}$

'Dr. Sami Ulus Maternity, Child Health and Diseases Training and Research Hospital, Ankara, Turkey; 'Başakşehir Pine and Sakura City Hospital, Istanbul, Turkey; ${ }^{3}$ Department of Orthopedics and Traumatology, Faculty of Medicine, Gazi University, Ankara, Turkey; ${ }^{4}$ Department of Physiotherapy and Rehabilitation, Faculty of Health Sciences, Yüksek ihtisas University, Ankara, Turkey; ${ }^{5}$ Department of Anatomy, Faculty of Medicine, Yüksek Ihtisas University, Ankara, Turkey

Objective: The flexion movement of the hand and fingers is carried out by the tendons extending from the forearm and palmar part of the hand to the fingers. The synovial and fibrous sheaths of the flexor tendons in the hand both stabilize the tendons during movements and allow sliding movements to occur without being stuck. Synovial sheaths wrap the tendons of the thumb and the other four fingers separately, allowing the tendons to slip during the flexion movement. Fibrous sheaths contain five annular (A1, A2, A3, A4, A5) and four cruciform scales $(\mathrm{C} 1, \mathrm{C} 2, \mathrm{C} 3, \mathrm{C} 4)$ that stabilize the tendons on phalanges. One of the most common pathologies seen as a result of using the hand in repetitive movements in daily life too much is "trigger finger". Trigger finger is a tendovaginitis that affects the hand flexor pulley system and is characterized by pain and dysfunction in the hand and fingers. As a result of thickening in the flexor tendon, tendon sheath, and especially in the A1 pulley, contractures may develop in the finger in the following periods, as well as stuck and pain during finger movements. Today, one of the most frequently used approaches in the surgical treatment of the trigger finger is the percutaneous release technique. It is very important to position the A1 pule on the palmar surface for the correct application of the percutaneous release technique. The purpose of our study is to determine the 
position of the A1 pulley on the palmar surface relative to the digital palmar fold.

Methods: Our study was carried out on 9 fresh upper extremity cadavers in the Anatomy Laboratory of the Yüksek İhtisas University. First of all, A1 pulleys of cadavers whose hand palmar surfaces were dissected by preserving digital palmar folds were made visible. Next, the distance between the digital palmar fold and the proximal of the A1 pulley and the length of the A1 pulley were measured for each finger. The measurements were carried out by the same anatomist, and each parameter was measured three times, and the average of three measurements was evaluated.

Results: As a result of our study, the average length of the A1 pulley was found to be $5.8 \pm 0.8 \mathrm{~mm}, 9.6 \pm 2 \mathrm{~mm}, 11.3 \pm 3 \mathrm{~mm}$, $7.8 \pm 1.8 \mathrm{~mm}$ and $7.7 \pm 3.4 \mathrm{~mm}$ in the first, second, third, fourth and fifth fingers, respectively. Mean distance between digital palmar fold and A1 pulley was found to be $7.3 \pm 3.2 \mathrm{~mm}, 19 \pm 2.3$ $\mathrm{mm}, 23.4 \pm 2.4 \mathrm{~mm}, 21.8 \pm 1.8 \mathrm{~mm}$ and $18 \pm 3 \mathrm{~mm}$ in the first, second, third, fourth and fifth fingers, respectively.

Conclusion: We think that our study results will help position the A1 pule on the palmar surface according to the digital palmar fold and thus facilitate the percutaneous release technique used in trigger finger treatment.

Keywords: A1 pulley, trigger finger, flexor tendon

\section{0-07}

Variations and clinical importance of superficial palmar arch in human fetuses

$\underline{\text { Solmaz E }}{ }^{1}$, Fazlıoğulları $Z^{1}$, Albay $S^{2}$, Ünver Doğan $N^{1}$, Karabulut $\mathrm{AK}^{1}$

'Department of Anatomy, Faculty of Medicine, Selçuk University, Konya, Turkey; ${ }^{2}$ Department of Anatomy, Faculty of Medicine, Süleyman Demirel University, Isparta, Turkey

Objective: It was aimed to examine the types of the superficial palmar arch (SPA) in human fetuses and to determine their variations.

Methods: Our study was conducted on 80 upper extremities of 40 formalin-fixed fetuses (19 males, 21 females) of 18-37 weeks of age, after the approval of the ethics committee of Selçuk University Faculty of Medicine for non-interventional clinical research (2019/33). A dissection microscope was used to protect the vascular and neural structures of the fetuses. The radial, ulnar, and median arteries were examined. It was evaluated whether there were palmar aponeurosis and tendon of the palmaris longus muscle structures. Types, variations, and common palmar digital arteries of SPA were visualized.

Results: SPA was found as a complete type in 58 hands $(72.5 \%)$ and an incomplete type in 22 hands $(27.5 \%)$. The median artery originating from the ulnar artery and passing through the carpal tunnel during its course was detected in two hands $(2.5 \%)$. It was determined as in the complete group, type A $47.5 \%$, type B $16.25 \%$, type C $3.75 \%$, type D $1.25 \%$, and type E $3.75 \%$, in the incomplete group, type F $15 \%$, type G $8.75 \%$, type H $2.5 \%$, and type I $1.25 \%$.

Conclusion: The awareness of SPA variations is important in interventions for arterial bleeding in the region, in hand microsurgeries such as vascular grafts and free flap applications. The presence of the median artery is valuable due to micro-evolutionary change or its decline and disappearance in terms of developmental anatomy and for neurologists and orthopedists, if it persists permanently, it is causing carpal tunnel syndrome by pressing on the median nerve in the aspect of clinical anatomy.

Keywords: superficial palmar arch, variation, fetus, median artery, developmental anatomy

\section{0-08}

\section{Variations and clinical significance of the superficial palmar arch: a cadaver study}

Uslu AI

Department of Anatomy, School of Medicine, SANKO University, Gaziantep, Turkey

Objective: The aim of this study is to examine the frequency of superficial palmar arch variations, to measure the diameters of the arteries participating into the arch structure and to evaluate bilateral symmetry.

Methods: This study was carried out in 18 hands of 9 formalin-fixed cadavers. The forming, branching and symmetry features of superficial palmar arch were examined using classical dissection method. The diameters of the ulnar artery, superficial branch of the radial artery and persistent median artery, which are included in the structure of the arch, were measured at the level of the wrist line connecting the processus styloideus radii and processus styloideus ulnae by using digital caliper. Number and percentage values were given as descriptive statistics.

Results: Superficial palmar arch was found complete in 11 (61.1\%) hands and incomplete in 7 (38.9\%) hands. Bilateral complete arch forming was found in four of these cadavers, and bilateral incomplete arch forming was found in two of these cadavers. A medioulnar type arch accompanied by persistent arteria mediana was detected in one hand of two cadavers without symmetry. Complete and incomplete arcs were classified according to Lippert and Pabst (1985). Accordingly, the radioulnar type complete arc, 50.0\% (n=9); medioulnar type complete arch, $11.1 \%(\mathrm{n}=2)$; incomplete type in which all common palmar digital arteries from the ulnar artery, $22.2 \%(n=4)$; The incomplete type in which the first common palmar digital artery originates from the radial artery and the others from the ulnar artery was found $16.7 \%(n=3)$. Arteria ulnaris was found as a dominant artery in all types. 
Conclusion: In the literature, there are studies reporting the incidence of the medioulnar type complete arch formed by persistent median artery and ulnar artery is found between $2.5-4 \%$, to up $15 \%$. In our study, we found a high rate of medioulnar type complete arch forming. We believe that keeping in mind the variations of such arch structures could be that of clinical importance in terms of correct diagnosis and treatment practices by radiologists and hand surgeons.

Keywords: superficial palmar arch, radial artery, ulnar artery, persistent median artery, variations.

\section{0-09}

Evaluation of the relationship between critical shoulder angle and acromial index measurements with rotator cuff rupture on 3D models

İnce $\mathrm{MS}^{1}$, Gözil R², Kanatlı $\mathrm{U}^{3}$, Bahçelioğlu $\mathrm{M}^{1}$

'Department of Anatomy, Faculty of Medicine, Gazi University, Ankara, Turkey; 'Department of Anatomy, Faculty of Medicine, Yüksek Ihtisas University, Ankara, Turkey; ${ }^{3}$ Department of Orthopedics and Traumatology, Faculty of Medicine, Gazi University, Ankara, Turkey

Objective: Since the identification of rotator cuff tears, many studies have been conducted to explain the causes of the tears. However, the relationship between rotator cuff tears and morphological features of acromion, cavitas glenoidalis and caput humeri has not been fully explained. The studies are usually done with two-dimensional radiography images. Because the positions of the joint faces could not be fully evaluated in twodimensional radiographic images, three-dimensional models were needed. The aim of this study is to evaluate the relationship between the rotator cuff tears and critical shoulder angle and acromial index values in three dimensions and to investigate the effects of critical shoulder angle and acromial index values on the formation of rotator cuff tears.

Methods: In our study, computerized tomography examinations of 44 participants, 24 rotator cuff tear (RMY group) and 20 Bankart lesions and no rotator cuff tear (control group) were examined. Critical shoulder angle and acromial index measured on glenohumeral joint models obtained by three-dimensional reconstructions of computed tomography examinations.

Results: In conclusion, rotator cuff tear group's critical shoulder angle was measured significantly higher than control group $(\mathrm{p}=0.002)$. Rotator cuff tear group and control group's acromial index values did not differ significantly $(\mathrm{p}=0.151)$. In addition, there was a moderate positive correlation between acromial index and critical shoulder angle values and age of patients with rotator cuff tears $(\mathrm{r}=0.309, \mathrm{r}=0.367)$.

Conclusion: Acromial index values were not found to be associated with rotator cuff tears unlike most of the two-dimensional radiographic evaluations, while critical shoulder angle values were found to be associated with rotator cuff tears in parallel with two-dimensional radiographic evaluations. We think that the reason for this difference may be that we performed our study on models obtained by three-dimensional reconstruction of computed tomography. We anticipate that our results will guide clinicians in revealing the etiology of rotator cuff degeneration and determining the surgical method to be used for rotator cuff repair.

Keywords: rotator cuff muscles, critical shoulder angle, acromial index, shoulder

\section{0-10}

Anatomical investigation of transverse fiber groups of the volar retinacular formation

Vural A ${ }^{1}$, Kaptan AY', Kanatlı $\mathrm{U}^{3}$, Aytaç $\mathrm{G}^{5}$, İnce MS ${ }^{4,5}$, Gözil R

'Dr. Sami Ulus Maternity, Child Health and Diseases Training and Research Hospital, Ankara, Turkey; ${ }^{2}$ Başakşehir Pine and Sakura City Hospital, Istanbul, Turkey; ${ }^{3}$ Department of Orthopedics and Traumatology, Faculty of Medicine, Gazi University, Ankara, Turkey; ${ }^{4}$ Department of Physiotherapy and Rehabilitation, Faculty of Health Sciences, Yüksek ihtisas University, Ankara, Turkey; ${ }^{5}$ Department of Anatomy, Faculty of Medicine, Yüksek ihtisas University, Ankara, Turkey

Objective: Palmar and digital fascia form an important stabilizing structure that provides some degree of protection of palms and fingers during movements. Palmar aponeurosis, which is part of the palmar fascia; It is roughly triangular and its beginning and has its beginning close to the palmaris longus tendon. As it moves away from the wrist, it divides into longitudinal strips or pretendinous bands that extend towards the fingers. In addition, aponeurosis palmaris has two groups of transverse fibers extending perpendicular to the pretendinous bands. The first of these is the superficial transverse palmar ligament (palmar aponeurosis' transverse palmar ligament) located at the level of the digital palmar crease. The second of the transverse fibers is the natatory ligament, which is more voluminous than the superficial transverse palmar ligament and extends at the level of the web spaces. The superficial fibers of the natatory ligament are connected to the flexor tendon sheaths and metacarpophalangeal joints, pointing on both sides. Dupuytren's contracture; It is a fibroproliferative disease of the palmar fascia of unknown etiology, which causes pathologically progressive contraction of the palmar fascia and flexion deformity of the fingers. Contracture is most often localized in the web spaces in the ulnar part of the hand. It is known that the mainly affected formation in Dupuytren's contracture is palmar aponeurosis. However, studies have shown that the transverse fibers of palmar aponeurosis, the natatory ligament, are affected by the disease process, while the superficial transverse palmar ligament does not participate in the disease. Therefore, selective aponeuroectomy in which the superficial transverse palmar ligament is preserved is recommended for the treatment of contracture. The aim of our study is to describe the morphological features of the superficial transverse palmar ligament and the natatory ligament, which are 
important in the surgical treatment of Dupuytren's contracture, and their relationship with each other.

Methods: Our study was carried out on 9 fresh upper extremity cadavers in the Anatomy Laboratory of the Yüksek İhtisas University. First of all, the superficial transverse palmar ligaments and natatory ligaments of the dissected cadavers were made visible. Next, the lengths and the distance between the superficial transverse palmar ligament and natatory ligament were measured for each web space. The measurements were carried out by the same anatomist, and each parameter was measured three times, and the average of three measurements was evaluated.

Results: As a result of our study, the average length of the superficial transverse palmar ligament was found to be $7.7 \pm 1.3 \mathrm{~mm}$, $8 \pm 1.7 \mathrm{~mm}$ and $7.4 \pm 1.4 \mathrm{~mm}$ at the second, third and fourth web spaces, respectively. The average length of the natatory ligament was determined as $6 \pm 1.3 \mathrm{~mm}, 7.3 \pm 1.5 \mathrm{~mm}$ and $5.7 \pm 0.9 \mathrm{~mm}$ at the second, third and fourth web spaces, respectively. The distance between the superficial transverse palmar ligament and natatory ligament was found to be $17.4 \pm 4.4 \mathrm{~mm}, 19.3 \pm 2.8 \mathrm{~mm}$ and $17.3 \pm 2.9 \mathrm{~mm}$ at the second, third and fourth web spaces, respectively.

Conclusion: According to our study results, we think that the positioning of the two ligaments relative to each other will guide the selective aponeuroectomy procedure performed in the surgical treatment of Dupuytren's contracture to protect the superficial transverse palmar ligament and to selectively remove the natatory ligament.

Keywords: natatory ligament, palmar aponeurosis, Dupuytren's contracture

\section{0-11}

The protection of the innervation of tensor fasciae latae in hip direct anterior and anterolateral approaches: cadaver study

Akkaya $\mathrm{M}^{1}, \underline{\text { Korkmaz AC }}{ }^{2}$, Güngör $\mathrm{Y}^{2}$, Gürsoy $\mathrm{S}^{1}$, Bozkurt $\mathrm{M}^{1}$, Açar, $\mathrm{HI}^{2}$

'Department of Anatomy, School of Medicine, Ankara University, Ankara, Turkey; ${ }^{2}$ Department of Orthopedics and Traumatology, Faculty of Medicine, Ankara Yıldırım Beyazıt University, Ankara, Turkey

Objective: We aim to get to the bottom of the superior gluteal nerve (SGN) injury in the anterior and anterolateral approaches of hip replacement surgery. In these approaches, we bring forward a proposal decreasing injury ratio of SGN.

Methods: On 22 formaldehyde fixed cadavers, we determined the points where SGN enter the fascia of gluteus minimus (GMin) (point A), exit this fascia or gluteal muscles (point B) and enter the tensor fasciae latae (TFL) (point C), respectively. We determined the locations of these points according to the anterior superior iliac spine (ASIS) and the greater trochanter (GT). In our study, we calculated the descriptive statistics such as mean, standard deviation, minimum and maximum values of measurements on the SPSS software package.

Results: In the anterior approach reaching up to the hip joint through the medial border of TFL, the distances between point A and ASIS were $40.40 \pm 11.50 \mathrm{~mm}$ vertically, $7.99 \pm 3.65$ $\mathrm{mm}$ horizontally. The distances between point A and GT were $17.76 \pm 13.57 \mathrm{~mm}$ vertically, $67.70 \pm 8.54 \mathrm{~mm}$ horizontally. The distances between point $\mathrm{C}$ and ASIS were $70.35 \pm 14.11 \mathrm{~mm}$ vertically, $11.74 \pm 6.61$. The distances between point $\mathrm{C}$ and GT were measured $13.00 \pm 7.92 \mathrm{~mm}$ vertically, $63.92 \pm 9.96 \mathrm{~mm}$ horizontally. On the ASIS-GM line in the anterolateral approach, the distance between SGN and ASIS was $35.71 \pm 9.62 \mathrm{~mm}$. On the half of cadavers, the SGN entered the fascia of GMin.

Conclusion: In the anterior approach of hip replacement surgery, SGN was about $4 \mathrm{~cm}$ from ASIS and entered the TFL at a distance of approximately $7 \mathrm{~cm}$ to the ASIS. The SGN could get damaged between these two points. In the anterolateral approach, the SGN was about $3.5 \mathrm{~cm}$ from ASIS on the ASISGT line. We think that considering these distances during surgical incision and insertion of retractors will reduce SGN damage.

Keywords: tensor fasciae latae, hip surgery, hip direct anterior approach, hip anterolateral approach, superior gluteal nerve

\section{0-12}

\section{Insall-Salvati ratio after anterior cruciate ligament} reconstruction with hamstring tendon autograft

$\underline{\text { Salkım B }}{ }^{1}$, Fazlıŏulları $Z^{1}$, Aydın BK ${ }^{2}$, Karabulut AK ${ }^{1}$, Ünver Doğan $\mathrm{N}^{1}$

'Department of Anatomy, Faculty of Medicine, Selçuk University, Konya, Turkey; ${ }^{2}$ Department of Orthopaedics and Traumatology, Faculty of Medicine, Selçuk University, Konya, Turkey

Objective: The extensor mechanism of the knee joint is linked to anterior cruciate ligament injuries. Various studies have been conducted on the patella and patellar tendon length, which are important components of this mechanism. This retrospective study aims to compare the length of the patellar tendon and Insall-Salvati ratio, which defines the patellar height, before and after anterior cruciate ligament reconstruction.

Methods: After the ethics committee approval (2020/349) for the research, 73 patients (mean age \pm standard deviation, $27.06 \pm 8.3$ years) who were diagnosed with anterior cruciate ligament tear between 2010-2020 and underwent arthroscopic anterior cruciate ligament reconstruction with hamstring tendon autograft were included. 67 patients were male and 6 were female. The patellar tendon length and patella length were measured from the preoperative and postoperative lateral knee radiographs of all patients, and the Insall-Salvati ratio was calculated.

Results: Patellar tendon length $(48.3 \pm 5.2 \mathrm{~mm}$ vs. $47.04 \pm 5.2$ $\mathrm{mm})$ and Insall-Salvati ratio $(1.02 \pm 0.14$ vs. $1 \pm 0.15)$ were found to be significantly greater before surgery than after surgery 
$(\mathrm{p}<0.01)$. When the right and left knee measurements were compared, postoperative patellar tendon lengths were found to be statistically significantly longer on the left side $(\mathrm{p}<0.05)$. When comparing among genders, patella lengths were found to be statistically $(\mathrm{p}<0.001)$ longer in men, while postoperative Insall-Salvati rates were greater in women $(\mathrm{p}<0.05)$.

Conclusion: This study showed that patellar tendon length is changed after arthroscopic anterior cruciate ligament reconstruction with hamstring tendon autograft. Considering the great effect of patellar tendon length on the mechanics of the patellofemoral joint, this result indicates that the load on the joint will increase.

Keywords: Insall-Salvati ratio, anterior cruciate ligament reconstruction, patellar tendon length, patellar height

\section{0-13}

Evaluation of axes and angular parameters by radiographic measurement in patients with pes planus diagnosis

$\underline{\text { Ekici } \mathrm{H}^{1}}$, Karabulut AK ${ }^{1}$, Fazlıogulları $Z^{1}$, Albayrak Gezer $\dot{\mathrm{I}}^{2}$

'Department of Anatomy, Faculty of Medicine, Selçuk University, Konya, Turkey; '2Department of Physical Medicine and Rehabilitation, Faculty of Medicine, Selçuk University, Konya, Turkey

Objective: Radiographic results have an important place in the evaluation and treatment plan of foot deformities. The aim of this retrospective study is to interpret the axes and angular parameters, which are frequently used in the radiographic evaluation of pes planus in the appropriate position, according to gender and age differences in adults.

Methods: Ethics committee approval was obtained for our study. In our study 50 patients (27 females, 23 males) diagnosed with pes planus between 2012 and 2019 at Selçuk University Faculty of Medicine (mean age $35.5 \pm 1.9 \mathrm{SD}$ ), since there were no data on healthy individuals in the comparison group, 50 patients (34 females, 16 males) who most frequently came to the clinic with the same complaints and were diagnosed with calcaneal spur (mean age 47.6 $\pm 1.7 \mathrm{SD}$ ) were included in the study. On the lateral and anteroposterior foot $x$-rays of all patients; calcaneal inclination, talohorizontal, lateral talo-first metatarsal, lateral talocalcaneal, anteroposterior talocalcaneal, talonavicular coverage, anteroposterior talo-first metatarsal angles were measured. In statistical preliminary analyzes, an independent $t$ test was used for data meeting the parametric assumptions. Chi-square test was used to compare cross tables of categorical groups. Correlations between parameters were examined. A p value $<0.05$ was considered significant.

Results: The mean calcaneal inclination angle in the pes planus group was $23.7^{\circ}$, in the control group $27.6^{\circ}$. The mean lateral talo-first metatarsal angle was $8.7^{\circ}$ in the pes planus group, $12.8^{\circ}$ in the control group. The mean lateral talocalcaneal angle was $42.4^{\circ}$ in the pes planus group, $43.8^{\circ}$ in the control group. All these angles showed statistically significant differences between the two groups. (respectively; $\mathrm{p}=0.006$, $\mathrm{p}=0.0001, \mathrm{p}=0.003$ ). According to gender differences; the mean lateral talo-first metatarsal angle is higher in women $\left(11.4^{\circ}\right)$ than men $\left(9.8^{\circ}\right)$, and the mean talo horizontal angle is higher in men $\left(19.0^{\circ}\right)$ than in women $\left(17.8^{\circ}\right)$. Differences in means were found to be statistically significant $(\mathrm{p}=0.001, \mathrm{p}=0.0001$, respectively). In both groups, it was observed that the talo horizontal angle was (-) correlated with age, and the talo horizontal angle decreased with increasing age $(\mathrm{r}=-0.259)$.

Conclusion: Radiographic axes and angles measured from the foot; It may be affected by gender and diagnosis differences. With the findings obtained, the method of measuring angles with radiography can be used in the evaluation of pes planus and calcaneahorizontal, lateral talus-os metatarsi I and lateral talocalcaneal angles can be considered to be more determinant.

Keywords: pes planus, angular parameters, foot $\mathrm{x}$-rays, calcaneal spur

\section{0-14}

\section{Effect of problems accompanying cerebral palsy on temporomandibular joint diseases}

Şahin Öztürkcan D ${ }^{1}$, Ünver Doğan N ${ }^{1}$, Kartal A², Karabulut $\mathrm{AK}^{1}$, Fazlıoğulları $\mathrm{Z}^{1}$

'Department of Anatomy, Faculty of Medicine, Selçuk University, Konya, Turkey; ${ }^{2}$ Department of Child Health and Diseases, Faculty of Medicine, Selçuk University, Konya, Turkey

Objective: The purpose of our study; to determine the relationship between problems accompanying CP and temporomandibular joint disorders (TMER) in adults and children with cerebral palsy (CP). Determining the underlying causes of existing pathologies will pave the way for preventive treatment planning.

Methods: 120 individuals with CP between the ages of 5-50 who were not previously diagnosed with TMER were included in the study. DC/TMD (Diagnostic Criteria/Temporomandibular Disorders) examination and survey criteria determined by the international RDC/TMD (Research Diagnostic Criteria/ Temporomandibular Disorders) consortium network were applied to the individuals. Problems accompanying individuals with SP in the survey criteria; questions and scales evaluating saliva problem, swallowing problem, nutritional characteristics of individuals, epilepsy problem, spasticity value and mental level were added.

Results: TMER was found to be significantly higher in individuals with $\mathrm{CP}$ who have swallowing problems compared to individuals without $(\mathrm{p}<0.05)$. Significantly higher TMER was found in individuals fed liquid and puree food compared to individuals without nutritional problems $(\mathrm{p}<0.01)$. TMER was found to be significantly higher in individuals with severe spasticity compared to individuals with mild spasticity $(\mathrm{p}<0.05)$. At 
least 1 TMER was found to be statistically significantly higher in individuals with mental retardation than those without symptoms.

Conclusion: The existence of problems accompanying SP poses a risk for TMER. As the severity of spasticity increases in individuals, the risk of TMER increases.

Keywords: cerebral palsy, temporomandibular joint, accompanying problems, anatomy

\section{0-15}

The effect of chronic respiratory diseases on ocular lens capsule structure: transmission electron microscope study

$\underline{\text { Dikmetas }}^{1}$, Firat A ${ }^{2}$, Kocabeyoğlu $\mathrm{S}^{1}$

'Department of Ophthalmology, Hacettepe University School of Medicine, Ankara, Turkey; ${ }^{2}$ Department of Anatomy, Hacettepe University School of Medicine, Ankara, Turkey

Objective: Single-layer lens epithelial cells under the anterior lens capsule are metabolically the most active part of the crystalline lens and serve as a metabolic motor that maintains the physiological health of the tissue. The cells of the lens epithelium regulate the most of the homoeostatic functions of the lens. The epithelium is the layer in which many of the mechanisms are regulated responsible for the transport of water, ions and nutrients through the lens. Tuberculosis (TB) is still seen frequently in Turkey. Extrapulmonary findings are expected in $20 \%$ of tuberculosis. Wegener's Granulomatosis (WG) is an autoimmune condition that affects the other important respiratory system. These patients can first present with their ocular findings and then receive their systemic diagnosis. The aim of this study was to evaluate the TEM findings in TB and WG patients by transmission electron microscopic (TEM) examination of the anterior capsule of the lens.

Methods: This was a prospective, interventional study. The research was approved by the Ethical Committee of the Hacettepe University Medical School (GO 20/405). Anterior lens capsules (basement membrane and lens epithelial cells) were taken from 10 eyes of 10 patients without systemic disease diagnosed with age-related cataract, 2 eyes of 2 TB patients, and 2 eyes of 2 WG patients. All patient and control groups were of similar age and had no additional systemic disease. Anterior lens capsules were obtained from cataract surgery and prepared for TEM. Thin sections of the tissue blocks obtained were cut with an ultramicrotome (LKB Nova, Sweden). These thin sections were stained with uranyl acetate and lead citrate, then examined under Jeol JEM 1200 EX (Japan) TEM.

Results: Significant ultrastructural changes were observed in the anterior lens capsules of all TB and WG eyes. Degenerative changes in lens epithelial cells were observed in all samples. In cases with WG, significant thinning and locally intracytoplasmic vacuoles were detected in lens epithelial cells compared to the control group. There was marked irregularity on the epithelial surface of the lens, and intercellular edema was observed under the lens capsule. In some areas, it was determined that the lens epithelium was distinctly separated from the capsule. In TB cases, interstitial and intracellular changes and basement membrane changes were found.

Conclusion: Transmission electron microscopy revealed ultrastructural abnormalities in the lens epithelium of all patients in the TB and WG groups, which were not seen in the control group. There are several studies in which degeneration and transduction of lens epithelial cells due to cataracts have been observed and discussed. Some changes seen in some types of cataracts (hypermature, traumatic, atopic) include swelling, cell loss, and degenerative lesions such as pyknotic nucleus or swelling. Patients with this type of cataract were excluded from this study. However, TB and WG are diseases in which many different departments work in coordination and many aspects are still uncertain, so more research is needed.

Keywords: Wegener's granulomatosis, tuberculosis, electron microscopy, lens capsule

\section{0-16}

\section{Evaluation of knowledge and attitudes of physicians} about cadaver donation in Turkey

Yeșil Kayabaşı C, Kurtoğlu Olgunus Z

Department of Anatomy, Mersin University Medical Faculty, Mersin, Turkey

Objective: Citizens who are interested in body donation frequently state that they apply to family physicians or physicians in hospitals first, instead of anatomy department, to get information. In this study, it was aimed to evaluate the knowledge and attitudes of physicians in Turkey about cadaver donation procedures.

Methods: This survey based descriptive study for physicians in Turkey was approved by Mersin University Clinical Research Ethics Committee (Approval number 2020/677). The questionnaire form consists of three parts and 20 questions. In the first part; demographic information such as age, gender, professional working time, field of study, In the second part; the items about the physicians' answers to frequently asked body donation questions, the donation form and the awareness of the physician responsibilities in the process; In the third part, whether the physicians have a request to be informed and their informing method preferences, are questioned. Anatomist physicians were excluded. The questionnaire was sent to the social media groups of physician professional associations and medical faculty graduate groups. The population of the study was determined according to the data of the Ministry of Health for the year 2020 on the number of physicians. Sample size was determined as 271 with $90 \%$ confidence. Descriptive statistics are expressed as percentages. 
Results: Of the 235 physicians from 32 different provinces (aged $25-68,58.7 \%$ female, $41.3 \%$ male) who accepted to fill out the questionnaire, $63.8 \%$ were specialist / specialty students, $36.1 \%$ were family physicians / general practitioners. $13.2 \%$ of the participants stated that at least one person applied to them for body donation. While only $52.8 \%$ of the physicians chose the Department of Anatomy to direct the applied donor candidate, $33.1 \%$ chose the Organ and Tissue Donation Units. $91.1 \%$ of the physicians had not examined a body donation form before. The rate of those who stated that the donation form could be approved by the physician in order to be a will was only $15.3 \%$. $80.4 \%$ of the physicians did not know that the body of an individual who did not fill out the donation form could be donated by their relatives, $29.8 \%$ did not know that body donation does not prevent organ donation, and 34\% did not know that the cadaver would have a grave later. $90.6 \%$ of the physicians stated that they needed to be informed about the laws and procedures regarding cadaver donation after graduation. In the question where more than one option could be marked for the method of notification, $65 \%$ of the participants stated that a website where they can access the relevant documents, $58.6 \%$ of them a document containing detailed information on the subject, $43.2 \%$ of them stated that the contact information of the authorities to whom they can apply for information when necessary can be benefited.

Conclusion: The study reveals that physicians do not have enough information about body donation, do not know how to guide citizens and need to be informed about this issue. We got the impression that sending a document containing information text, electronic information addresses and anatomist contact information to physicians will meet the demand and increase awareness. In addition, we suggest that adding cadaver donation and legal procedures to medical education programs before graduation will contribute to the long-term solution of the weaknesses.

Keywords: cadaver donation, body donation, physician, attitude, survey

\section{0-17}

Information on body donation promotion at official web pages of anatomy departments: a qualitative evaluation

Ok F, Gürses İA

Department of Anatomy, Istanbul Faculty of Medicine, Istanbul University, Istanbul, Turkey

Objective: Raising the public awareness on body donation is crucial for countries that have struggling donation programs to improve body donation numbers. The official web pages of anatomy departments may contribute to achieve this goal. Nevertheless, previous studies reported institutional or individual reluctance of departments or anatomists, respectively, due to an expectation of adverse publicity. This study aimed to investigate whether the official web pages of Turkish anatomy departments provided information on body donation and analyze the content of provided information.

Methods: Official web pages of anatomy departments at one hundred medical faculties were evaluated regarding how much information was provided on body donation. Texts of information and all documents provided at the web pages were collected. Gathered data was evaluated qualitatively with a six step latent thematic analysis in order to outline the range of information and detect any existing common themes among the data on body donation.

Results: Twelve departments (12\%) provided information on body donation with varying content. Thematic analysis revealed five main themes regarding content of information. These were (1) legal aspects, (2) significance, (3) acceptance/rejection criteria, (4) availability, and (5) redirection. All departments $(n=12$, $100 \%)$ outlined the legal aspects of body donation, 7 departments $(58 \%)$ highlighted the significance of body donation, 7 departments $(58 \%)$ provided contact information in case of possible donations, 5 departments (41\%) covered acceptance/rejection criteria, and 3 departments (25\%) provided a link to the official website of the Turkish Society of Anatomy and Clinical Anatomy.

Conclusion: In addition to low donation registrations and inadequate body donations, the number of anatomy departments that provide adequate information on body donation on their websites was very low. Additionally, the content of provided information varied among departments. Encouragement, coordination, and participation of anatomy departments to existing awareness activities via their official web pages seem necessary. We believe that the themes and subthemes gathered with the qualitative analysis in this study could be used as a template for information to be shared through departmental web pages.

Keywords: anatomy education, anatomy departments, anatomy web pages, body donation programs, body donation promotion

\section{0-18}

New era in body donation; contactless body donation Ortadeveci A, Özden H

Department of Anatomy, Faculty of Medicine, Eskişehir Osmangazi University, Eskişehir, Turkey

Objective: With the development of technology, people carry out many activities and tasks from their home or office via the internet. In this context, the first serious change has been experienced in the commercial field and has started to spread rapidly to all areas over time. The COVID-19 pandemic that shook the whole world with the beginning of 2020 has taken people away from social environments and made it necessary to come up with new alternatives to receive all services remotely. 
The education and science world has also had to keep up with these changes and the adaptation process is still ongoing. One of the chronic problems of Anatomy education in our country is the difficulties in obtaining cadavers due to the insufficient number of donations. Taking into account the average age of donors and the risks and concerns about leaving the house, cadaver donation from a distance will create a more comfortable and practical situation for donors. Our aim in this study is to increase the number of cadaver donations in our country and to transform the changes experienced during the COVID-19 pandemic process into a permanent advantage.

Case: After the COVID-19 pandemic, scientists have invited people to move away from social environments and to minimize contact as much as possible. In this process, first curfews were applied to certain age ranges and then the same bans were applied for all citizens on some days. As such, cadaver donations were also interrupted due to difficulties in carrying out official procedures. During this period, a 55-year-old male volunteer contacted Eskişehir Osmangazi University Anatomy Department and stated that he wanted to donate his body as a cadaver. The donor was asked to wait for a while and then the situation was evaluated within the department and it was agreed that the execution of normal donation procedures would not be in accordance with the current health measures. For this purpose, the official documents required to be filled for cadaver donation were sent to the volunteer via e-mail and the donation procedures were completed with the same method. Due to the age of the donor, this type of communication was only possible through their relatives, and this practice had a negative impact on the donor, donor relatives and the donation process. In addition, it was thought that filling out official documents by e-mail might lead to security concerns. As a result of this case, our research team examined the literature in order to apply more reliable and more easily applicable methods in cadaver donation, and tried to find the most ideal method in terms of reliability, ethical principles and efficiency among the methods used and candidates for use.

Conclusion: In order to increase the number of cadaver donations in our country, it will be a necessity in the near future to reach more people and to switch to an online infrastructure where official procedures are made easier. In recent years, many formal transactions can be done remotely or online via www.turkiye.gov.tr. Our recommendation is to create an infrastructure on this site that will enable cadaver donation and tracking. If such a system is applicable, it will be an easy and highly reliable method. Because it is predicted that both personal wet signatures and fingerprints will take place in this system in the near future. Updating ourselves at any time and adapting to innovations in order to increase the number of cadaver donations in our country will make it easier to overcome the difficulties that may be encountered.

Keywords: donor, cadaver, donor comfort, remote cadaver donation

\section{0-19}

How helpful can scientific research be for generalizing body donation in Turkey?

\section{Gürses İA}

Department of Anatomy, Istanbul Faculty of Medicine, Istanbul University, Istanbul, Turkey

In Turkey, both body donation registrations and body donations are infrequent. Although awareness activities within the last decade managed to significantly increase body donations, this increase is far from supporting the educational and research activities at institutions. How to increase the efficiency of awareness activities and improve body donation practice is still under debate. Conducting and planning research studies that collect reliable sectional and prospective scientific data on body donation might help to solve existing cadaver problem. National research studies performed within the last two decades have gathered invaluable information regarding body donation practice. These studies had investigated current cadaver sources, characteristics of cities that body donation was possible, attributes and motivations of individuals who registered as body donors, annual changes in body donation registrations, support of anatomy departments to awareness activities, and how awareness activities had an effect on the general population. The findings of these studies had an impact on many areas related to body donation. It was seen that body donations were possible in cities with higher educational and economic statuses and donation registrations increased significantly since 2012 on a national level. Studies also showed that the retention time of bodies varied among donors, how their personal information could be shared, and that there is a need to update current donation forms regarding the current legislature. Additionally, studies outlined how donor motivations could be used as a baseline for awareness activities and to recruit new body donors. Nevertheless, the average donor profile concealed existing sub-profiles, therefore, anatomists now have new responsibilities due to the existence of these sub-profiles. Finally, these studies emphasized which media could be used for awareness activities, how much anatomy departments supported awareness activities through identified media, the increase in public awareness on body donation did not match the awareness on where to apply for an official donation, a temporal planning for awareness activities is possible, and how to identify gradual target audiences for awareness activities. Applying the results of these studies to daily practice might be preferred in order to disperse existing awareness activities on a national level and improve the efficiency of awareness activities. Planning new research studies might also help us to detect and correct possible problems observed in daily practice. Therefore, encouraging young anatomists to conduct novel research studies focusing on the acquisition of reliable scientific data on body donation seems important for attaining a more prevalent body donation practice.

Keywords: cadaver, body donation, body donation awareness, scientific research, evidence based medicine, Turkey 


\section{0-20}

Association of major anatomical structures of the thorax with surface anatomy in children

Şener U ${ }^{1}$, Tellioğlu Metin A ${ }^{1}$, Durum Polat $\mathrm{Y}^{2}$

${ }^{1}$ Department of Anatomy, School of Medicine, Aydın Adnan Menderes University, Aydın, Turkey; 'Department of Radiology, School of Medicine, Aydın Adnan Menderes University, Aydın, Turkey

Objective: Pediatric anatomy is different from adults. To perform clinical procedures safely, it's essential that knowledge on surface anatomy should be accurate and age-standardized. Hence the aim of the study was to evaluate the association between major structures and surface landmarks of thorax in children.

Methods: Computer tomography scans of 127 patients, aged, $0-18$, without thoracic pathology were evaluated. Patients were divided into five groups according to the age. Ethical approval for this study was obtained from Aydın Adnan Menderes University Noninterventional Clinical Trials Ethics Committee (2019/15). Surface anatomy of sternal angle (AS), central veins, cardiac apex, domes and openings of diaphragm were defined according to corresponding vertebrae, ribs, costal cartilage (CC), intercostal space (SI) level. Vertical distance between AS plane and aortic arch, azygos vein-superior vena cava (SVC) junction, bifurcations of trachea and pulmonary trunk were measured. Structures situated $1 \mathrm{~cm}$ distance from the AS plane were accepted as being within the plane. Distances of cardiac apex to midline (LMA) and midclavicular line (LMC) were measured.

Results: AS plane was at T4 and T5 levels. Azygos vein-SVC junction and bifurcation of trachea were found within the AS plane. Aortic arch was observed above the AS plane, bifurcation of the pulmonary trunk was below of it. Both brachiocephalic veins formed lateral to sternoclavicular joints in younger groups, while it was posterior to it in olders. Formation of SVC in adolescents was at CC I but it was at sternoclavicular joint level in 4-7 and 8-11 age groups. SVC-right atrium junction was at SI II-CC III. Cardiac apex was at SI V in adolescents and at Sİ IV-rib $\mathrm{V}$ in younger ages. Apex-LMC distance was less than $1 \mathrm{~cm}$ in all groups. Apex-LMA distance ranged between $4.7-7.6 \mathrm{~cm}$. Domes of diaphragm on both sides were at SI IVSI V level. Caval opening was observed at lower than T8 and aortic hiatus was at T12 in all age groups. Esophageal hiatus was above T10 in 0-4 and 4-7 age groups.

Conclusion: Although the level of AS in children was found similar to adults, structures within AS differs from adult studies. Midclavicular line is an appropriate surface line for cardiac apex. Central veins in our population were situated in higher positions, in comparison with adult and pediatric studies in different populations. Age and ethnicity lead variations in surface anatomy. We conclude that our findings will contribute to physical examination of the heart, diagnosing diaphragmatic eventrations, invasive procedures like central venous catheterisation and pleural resection/biopsies.

Keywords: surface anatomy, pediatric anatomy, thorax, radiologic anatomy

\section{0-21}

The effect of tracheal diverticulum and chronic obstructive lung disease on chest anthropometry

Argalı Deniz $\mathrm{M}^{1}$, Er Ulubaba $\mathrm{H}^{2}$, $\operatorname{Arpacı}_{\mathrm{MF}^{3}}$, Çavuş $\mathrm{F}^{3}$, Denirtaş $\mathrm{G}^{4}$, Karataş $\mathrm{T}^{3}$, Özbağ $\mathrm{D}^{3}$

'Department of Physical Therapy and Rehabilitation, Süleyman Demirel University Research and Practice Hospital, Isparta, Turkey; ${ }^{2}$ Department of Radiology, Yeşilyurt Hasan Çalık Government Hospital, Malatya, Turkey; ${ }^{3}$ Department of Anatomy, Medicine Faculty, Inönü University, Malatya, Turkey; ${ }^{4}$ Malatya Research and Practice Hospital, Malatya, Turkey

Objective: Tracheal diverticulum (TD) cases are usually asymptomatic and therefore their frequency may be overlooked. Its incidence varies according to the type of radiological examinations performed. In this study, chest anthropometries of Chronic Obstructive Pulmonary Disease (COPD) patients with TD and those without COPD but with TD were examined. Our aim was to observe the effect of TD on chest anthropometry.

Method: After ethical permission we have investigated 995 patients between 40-80 age who underwent thorax computerized tomography (CT) between January 2019 and March 2020 retrospectively. Axial, coronal and sagittal planes were used for measurements, 31 of 995 patients had TD. The localization, size, vocal cord, distance to hull, and level of all detected TDs were noted. In addition, chest diameters at T4 and T9 levels were measured as transverse and anteroposterior. These patients were divided into two separate groups as Group 1 only TD and Group 2 TD + COPD. Group 3 and 4 were formed from the same age range and same sexes as Group 3 only COPD and Group 4 healthy individuals with the thorax CTs of the same dates. The relation of chest diameters with TD and COPD was examined in all groups. Independent student's ttest was performed for statistical analyzes.

Results: While there was a statistically significant difference in the vocal chord distance of TD in Group 1 and Group 2, no significant difference was found in the distance to the carina. While the average distance of TDs of both groups to the vocal chord is $5.75 \pm 1.57$, the average distance to the carina is $7.02 \pm 2.36$. There was no statistically significant difference in transverse and vertical size of TD in Group 1 and Group 2. The average transverse size of TDs in both groups is $6.63 \pm 3.04$, while the average vertical size is $19.1 \pm 7$.24. It was observed that the vertebral levels of TDs in Group 1 and Group 2 were mostly at the T2 and T3 levels. When the average transverse and vertical diameter lengths of all groups at T4 
and T9 levels were examined, a statistically significant difference was found between Group 1 and Group 3 ( $\mathrm{p}=0.016)$ $(\mathrm{p}=0.017)$ only in the vertical diameter length at the T4 and T9 levels.

Conclusion: In this study, we observed that the TD levels of COPD patients were mostly at the T2-T3 level and COPD had no effect on TD dimensions, only affecting the level at which the diverticulum is located. We also found that TD has a reducing effect on anteroposteriorly chest parameters on COPD patients.

Keywords: diverticulum, COPD, tracheal diverticulum, thoracentropometry

\section{$0-22$}

Assessment of patent foramen ovale and its clinical significance in patients with migraine with aura

Huyut MA

Department of Cardiology, Faculty of Medicine, Istanbul Yeni Yüzyil University, Istanbul, Turkey

Objective: Patent foramen ovale (PFO) is a congenital anomaly with a frequency of $25-30 \%$ in the general population. It has been determined in studies that the incidence of PFO is increased in patients with migraines, and studies are indicating that the frequency of PFO has increased especially in migraine patients with aura. This study aims to investigate whether there is a relationship between PFO and total headache duration in patients diagnosed with migraine with aura.

Methods: Transesophageal echocardiographic findings of 46 consecutive patients diagnosed with migraine with aura, between January 2020 and June 2020 were analyzed. The presence of interatrial septal aneurysm (ASA), interatrial septal defect (ASD), or interatrial PFO anomalies was recorded. ASD in 1 patient and ASA in 5 patients were detected and excluded from the study. It was recorded that there were 40 patients with PFO. We collected data such as in-hospital migraine attacks, how many migraine attacks were seen in a month, and the duration of headache when migraine developed, past medical history, and laboratory data of patients. The study was conducted in accordance with the Helsinki declaration of principles and the ethics of good clinical practice. The data obtained in the study were evaluated using SPSS 22.0 (statistical software, SPSS Inc., Chicago, IL, USA) package computer program, and the significance was evaluated at $\mathrm{p}<0.05$ level.

Results: 13 of the patients with migraine with aura were male (32.5\%), 27 were female (67.5\%), and mean age was $42.06 \pm$ 14.74 years. PFO was detected in $18(45 \%)$ of the patients. 14 of the patients with $\mathrm{PFO}$ were found to be female (77.77\%). When patients with $\mathrm{PFO}$ were compared with patients with migraine without aura, it was found that the rates of migraine attacks in the hospital were similar $(\mathrm{p}=0.457)$. While an average of $3.16 \pm 0.44$ attacks per month was detected in patients with PFO, $1.14 \pm 0.54$ migraine attacks with aura were found in the non-PFO group $(\mathrm{p}=0.032)$. Also, it was found that the duration of the attack was prolonged in patients with $\mathrm{PFO}$, while the attacks lasted an average of $36.14 \pm 9.28$ minutes in patients with $\mathrm{PFO}$, while it was found to last $21.58 \pm 7.64$ minutes in the nonPFO group ( $\mathrm{p}=0.041)$.

Conclusion: In conclusion, statistically significant more migraine attacks and longer attack durations are detected in patients with PFO, and investigation of PFO anomaly in migraine patients with aura is important in terms of estimating the clinical course of the patients.

Keywords: migraine with aura, migraine, patent foramen ovale, transesophageal echocardiography

\section{0-23}

The association of systolic pulmonary artery pressure with pulmonary artery diameter, age, disease duration, stage of disease and extrapulmonary involvement in patients with sarcoidosis

\section{Korkmaz C}

Department of Chest Diseases, Meram Faculty of Medicine, Necmettin Erbakan University, Konya, Turkey

Objective: Pulmonary hypertension $(\mathrm{PH})$ is a well-known complication of sarcoidosis, associated with a significant increase in mortality. The number of studies investigating the association between $\mathrm{PH}$ and pulmonary artery diameter (PAD) is limited. In our study, we aimed to investigate the association of systolic pulmonary artery pressure (sPAP), which is one of the routine echography $(\mathrm{ECHO})$ data still providing valuable information in the diagnosis of $\mathrm{PH}$, with pulmonary artery diameter (PAD), age, gender, duration of disease, stage of the disease, extrapulmonary involvement, and spontaneous remission.

Methods: Sixty sarcoidosis patients diagnosed and followed-up in our chest diseases clinic between 2010 and 2020 were included in our study. An approval was obtained from the ethical board of the institution, and patients' demographic data, clinical course, information over treatment, radiological and echocardiographic examinations were recorded. $\mathrm{SPAB}$ was utilized to calculate mean PAP based on ECHO (average $\mathrm{PAP}=0.61$ xsystolic $\mathrm{PAP}+2 \mathrm{mmHg}$ ), and mean PAP was accepted as $\geq 25 \mathrm{mmHg}$ PH. PAD was measured by an experienced radiologist in thoracic CT. While the Pearson's correlation test was used in statistical analyses, the Spearman's correlation test was utilized for the variables not showing normal distribution. The student's T-test for comparisons between groups and the Mann Whitney $\mathrm{U}$ test for non-normally distributed variables were also used.

Results: A moderately strong positive significant correlation was found between $\mathrm{PAP}$ and PAD $(\mathrm{r}=0.46, \mathrm{p}<0.001)$. PAD was 
found significantly higher in patients with $\mathrm{PH}$, compared to those without $\mathrm{PH}(39.7 \pm 4.6$ vs $27 \pm 4.9)$. sPAP was also found significantly higher in those without extrapulmonary involvement than those with extrapulmonary involvement $(31.03 \pm 6.35$ vs $27.52 \pm 3.77, \mathrm{p}=0.01$ ).

Conclusion: There was a moderately strong positive correlation between SPAP and PAD in sarcoidosis patients, and the correlation was found to be significantly higher in patients with $\mathrm{PH}$ than those without PAD. This also shows that PAD, which can be easily measured during thoracic CT examination in patients with sarcoidosis, can be a guide in predicting sarcoidosis-related pulmonary hypertension (SRPH). Also, the fact that sPAP was found significantly lower in patients with extrapulmonary involvement gives an idea for more comprehensive studies including right heart catheterization (RHC) to be used as an indicator of good prognosis in such patients.

Keywords: pulmonary artery diameter, pulmonary hypertension, sarcoidosis, systolic pulmonary artery pressure

\section{0-24}

Is there a relationship between the amount of abdominal fat and kidney stone disease?

Kadiyoran C, Yilmaz PD

Department of Radiology, Meram Faculty of Medicine, Necmettin Erbakan University, Konya, Turkey

Objective: Although the incidence and prevalence of kidney stone disease has increased in recent years, kidney stones are seen in $10-12 \%$ of men and $5-6 \%$ of women. Certain risk factors have been identified, and metabolic causes such as obesity, hyperglycemia, hyperuricemia, and hyperlipidemia also play a role in the etiology, as well as reasons such as age, gender, genetic factors, race caused by life in different geological regions, dietary habits, and climatic differences. In our study, we aimed to investigate the relationship between the amount of intraabdominal fat (IAF) and kidney stone disease using sections obtained by computed tomography.

Methods: Patients who are admitted to our radiology unit from various polyclinics are required to have a non-contrast abdominal computed tomography (CT) examination for urinary system stone disease, and these images are performed with a Siemens Drive 256-slice CT device. The first group was formed of patients with kidney stones on CT by scanning the hospital radiological imaging system. The control group consisted of patients who presented with a suspicion of stones but any stone detected by CT examination. 54 patients were included in the patient group with stones detected on CT and 48 patients in the control group without stones were included. IAF measurements of the patients were calculated by Siemens Via workstation in a single axial section at the level of umbilicus in order to provide a standard in all patients, and the values obtained for both groups were noted and compared. Statistical analysis was performed with SPSS Version 20.0. Whether the data showed a homogeneous distribution was evaluated with the Kolmogorov-Smirnov test. Categorical data were evaluated by the chi-square and fisher-exact test. Homogeneously distributed data were evaluated by Student-t-test and nonhomogeneous data were evaluated by the Mann-Whitney U test. A p-value $<0.05$ was considered significant.

Results: The mean age was $48.09 \pm 14.3$ year in the patient group, and $47.1 \pm 11.2$ year in the control group. There is no significant difference between age and gender distributions in both groups. In the patient group with kidney stones, the mean value of IAY was $180 \pm 24 \mathrm{~cm}^{2}$, and in the control group, the mean value of IAF was $137 \pm 14 \mathrm{~cm}^{2}$. When the values calculated for both groups were compared, it was determined that the amount of IAF was higher in the patient group with stones, compared to the control group without stones, and the difference was statistically significant $(\mathrm{p}=0.022)$.

Conclusion: The relationship between obesity, metabolic syndrome and renal stone is mentioned in previous studies. A link between the amount of intraabdominal fat and renal stone disease was also reported, and it correlated with the result of our study. Studies with high patient populations have reported a clear link between obesity and urolithiasis. Additionally, the increase in the percentage of intraabdominal adipose tissue is associated with recurrent kidney stone formation. Similarly, in our study, it is understood that there is a strong correlation between the amount of IAF and kidney stone formation. There are many known reasons in the etiology of kidney stone disease, whose incidence and prevalence has increased in recent years. In our study, we concluded that the amount of intraabdominal fat may be a new risk factor for the development of kidney stone disease.

Keywords: intraabdominal fat, renal stone, computed tomography

\section{0-25}

Reappraisal of the types of trigeminal porus and importance in surgical applications: a cadaveric study

Öğüt $\mathrm{E}^{1}$, Armağan $\mathrm{K}^{2}$, Barut $\mathrm{Ç}^{1}$

'Department of Anatomy, School of Medicine, Bahçeşehir University, Istanbul, Turkey; ' 2 School of Medicine, Bahçeşehir University, Istanbul, Turkey

Objective: The detailed information regarding the types of trigeminal porus (TP), and related surgical approach is lacking in the literature. Therefore, we performed this study to elucidate further the types of TP and the relationships with critical surgical landmarks in the skull base.

Method: The study was performed on 19 formalin-fixed cadavers of the cranial base $(52.6 \%$ male, $n=10 ; 47.4 \%$ female, $n=9)$ on both sides. It was approved by the ethics committee of Bahcesehir University, Istanbul, Turkey on 18 March 2020 
with protocol number 2020-05/02. Calculations were made of the length, width, and types of TP, the thickness of the TP, the width and length of the internal acoustic meatus (IAM), the distance between the TP-IAM, the thickness of the ossifying tissue that forms the $\mathrm{TP}$, the trigeminal nerve $(\mathrm{CN} \mathrm{V})$ in both types, and the distance between the CN V-VI. The chi-square test was used for comparison of types, independent sample ttest and Mann Whitney $U$ test for comparison of distances by gender, paired t-test and Wilcoxon were used for comparison of parameters by body side.

Results: Elliptical (42.1\% left, 36.8\% right) and oval (52.6\% left, $36.8 \%$ right $)$ types of TP were detected $\left(\chi^{2}=11.722\right.$, $\mathrm{p}>0.05)$. The width of the TP was, on average, $8.02 \mathrm{~mm}$ (female) and $9.2 \mathrm{~mm}$ (male) on the right side, and $8.26 \mathrm{~mm}$ (female) and $8.81 \mathrm{~mm}$ (male) on the left side. The length of the TP was, on average, $1.99 \mathrm{~mm}$ (female) and $2.65 \mathrm{~mm}$ (male) on the right side, and $2.42 \mathrm{~mm}$ (female) and $2.94 \mathrm{~mm}$ (male) on the left side. The thickness of the ossifying tissue that forms the TP was $4.8 \mathrm{~mm}$ (female) and $4.7 \mathrm{~mm}$ (male) on the right side, and $5.09 \mathrm{~mm}$ (female) and $5.58 \mathrm{~mm}$ (male) on the left side. The distance from the TP to the IAM was $6.6 \mathrm{~mm}$ (female) and 6.13 $\mathrm{mm}$ (male) on the right side, and $6.21 \mathrm{~mm}$ (female) and 6.77 $\mathrm{mm}$ (male) on the left side. The distance from the CN V to CN VI was $6.76 \mathrm{~mm}$ (female) and $6.97 \mathrm{~mm}$ (male) on the right side, and $6.45 \mathrm{~mm}$ (female) and $7.04 \mathrm{~mm}$ (male) on the left side.

Conclusion: Identification of the trigeminal porus and detailed knowledge about its types and distance to the nearby surgical landmarks play a vital role in the treatment of trigeminal neuralgia and neuropathy due to the compression of the nerve. Measurements and types given in the current study may be of use in planning and choosing appropriate surgical approaches in the skull base.

Keywords: trigeminal porus, porus trigeminus, trigeminal nerve, skull, skull base

\section{0-26}

\section{Morphometric development of medial surface of} cerebrum in fetal cadavers

Şanlı OC, Öztürk K, Dursun A, Kastamoni Y, Albay S Department of Anatomy, Faculty of Medicine, Süleyman Demirel University, Isparta, Turkey

Objective: In our study, it was planned to investigate the size development of these measurements during gestational age (months), whether there is a difference between genders and right-left sides by making morphometric measurements related to the medial surface of the cerebrum in fetal cadavers.

Methods: Our study was carried out on 80 cerebral hemisphere of 40 fetuses (20 males, 20 females) with no external anomalies and pathologies, between the ages of 22-40 weeks of gestation, in the Department of Anatomy of the Faculty of
Medicine, Süleyman Demirel University. Fetuses were divided into 5 groups as between 22-24 weeks of age 6th month, 25-28 weeks 7 th month, 29-32 weeks 8 th month, 33-36 weeks 9th month, 37-40 weeks term. After the calvaria and falx cerebri were excised, the two cerebral hemispheres were separated with a median incision, and the dura mater and arachnoid mater were removed carefully. Then the medial surfaces of the cerebrum were photographed parallel to the ground. The measurements were made by uploading the photographs taken to the Image-J program. Height of cerebral hemisphere, anteroposterior length and total area of medial surface of cerebrum were measured. The development of these parameters during gestational age (months) was evaluated, and a comparison was made between right and left sides and genders. In addition, the weeks when sulcuses started to be seen were determined. The data obtained were analyzed with SPSS 20.0 for Windows program.

Results: It was determined that the dimensions of the measured parameters increased significantly with gestational age. When these parameters were examined between the right-left sides and genders, no statistically significant difference was found ( $>0.05)$. According to our study, sulci in the medial surface of the cerebrum had been formed since calcarine sulcus 23-24th, cingulate sulcus 24-25th, marginal sulcus 27-28th, paracentral sulcus 29-30th, parolfactory sulcus 30-31st, subparietal sulcus 30-31st weeks. Parieto-occipital sulcus was detected in all fetuses we dissected from the 22nd week.

Conclusion: According to the literature reviews we conducted, we did not find such a morphometric study in fetal cadavers. Our study will make important contributions to the literature on the morphology and morphometry of the medial surface of the cerebrum in the fetal period. Knowing the anatomy of the cerebral cortex and learning its development during the fetal period can play a role in the early diagnosis of developmental anomalies in the brain. Our study will be a reference for future studies.

Keywords: Image J, dissection, cerebral sulci, fetus

\section{0-27}

The evaluation of the changes in fossa cranii posterior and cerebellum in patients with Chiari type 1 malformation

$\underline{\text { Bağc1 G }}{ }^{1}$, Karabulut $\mathrm{AK}^{2}$, Batur A ${ }^{3}$, Fazlıŏgulları $Z^{2}$, Ünver Doğan $\mathrm{N}^{2}$

'Department of Anatomy, Faculty of Medicine, Kütahya Health Sciences University, Kütahya, Turkey; '2Department of Anatomy, Faculty of Medicine, Selçuk University, Konya, Turkey; ${ }^{3}$ Department of Radiology, Faculty of Medicine, Selçuk University, Konya, Turkey

Objective: Chiari malformation (CM) is a malformation that is mostly congenitally seen as a result of the structures in the fossa cranii posterior (FCP) being herniated through the foramen 
magnum towards the canalis vertebralis. Chiari type 1 malformation (CM1) is the simplest form of CM and defined only as the displacement of the tonsilla cerebelli at least $5 \mathrm{~cm}$ below the foramen magnum. Although its etiology is not known exactly today, it is thought that the insufficiency in the volume of FCP during the development of the cranium may be the cause. In this study, we aimed to retrospectively evaluate the effect on changes in FCP dimensions in the etiology of the disease by measuring FCP and cerebellum morphometry.

Methods: In our study, 50 patients diagnosed CM1 by radiologists working in the radiology department of Selçuk University Faculty of Medicine besides 50 control group participants who were admitted to our hospital with headache and similar complaints, cranial MRI scans were performed, but no pathological findings were found. Control group participants were selected demographically homogeneous to patients with CM1. In order to minimize age-related changes in cranium, individuals under the age of 18 and over the age of 65 were excluded from the study. McRae line, Klaus index, clivus length, supraocciput length, FCP diameter, FCP height, Welcher basal angle, Boogard angle, nasion-basion-opistion (NBO) angle measurements were performed from the length and angle measurements used to evaluate FCP morphometry. In addition, cerebellum lateral diameter, cerebellum height and degree of tonsillar herniation were measured. Statistical analysis of the data was performed using the SPSS 25 program. The suitability of quantitative variables for normal distribution was studied using the Shapiro Wilk test. Mann Whitney U test was used to compare variables that don't show normal distribution assumptions. Results with a p value of 0.05 were considered statistically significant.

Results: The mean age of the group of patients with CM1 was $37.96 \pm 11.22$ and the mean age of the control group was $38.20 \pm 12.28$. In the group of patients with CM1, Welcher basal angle, Boogard angle, NBO angle, cerebellum height were increased compared to the control group, clivus length, klaus index, supraocciput length and FCP height were decreased compared to the control group. These increase and decrease values were statistically significant $(\mathrm{p}<0.05)$. Although McRae line length was found to have increased slightly in the CM1 group, this increase was not statistically significant. FCP diameter and cerebellum lateral diameter were close to each other in both groups and were not statistically significant.

Conclusion: Our morphometric measurements show that FCP dimensions in CM1 patients are smaller than healthy people. We think that our results will contribute to the up-to-date anatomical knowledge of morphological measurements in FCP sizes of CM1 patients.

Keywords: Chiari malformation, Chiari type 1 malformation, fossa cranii posterior, magnetic resonance imaging, anatomy

\section{0-28}

Evaluation of cerebellar lobular anatomy in pediatric population

$\underline{\text { Isıklar S }}^{1,2}$, Özdemir S ${ }^{2}$, Demir $\dot{I}^{1}$, Özpar R

'Health Services Vocational School, Medical Imaging Techniques Program, Bursa Uludağ University, Bursa, Turkey; ${ }^{2}$ Department of Anatomy, Medical Faculty, Bursa Uludağ University, Bursa, Turkey; ${ }^{3}$ Department of Radiology, Medical Faculty, Bursa Uludağ University, Bursa, Turkey

Objective: The cerebellum, which plays a role in coordinating many vital functions from speech and balance to eye movement, consists of the corpus medullare and the cortex cerebelli surrounding it. Cortex cerebelli consists of lobules named from I to X. Cerebellar functions are topographically organized and tend to be localized to specific regions, as in the cerebrum. This study investigated age and sex differences in cerebellar lobular anatomy in pediatric population.

Methods: Pediatric individuals (0-18 years old) who underwent cranial magnetic resonance imaging between 2012-2020 in the Department of Radiology, Faculty of Medicine, Bursa Uludağ University, were retrospectively analyzed. Out of 985 patients who underwent 3D-T1-weighted volumetric imaging, 485 individuals (258 Male, 227 Female) who were evaluated as radiologically normal were included in the study. Persons with any cerebral or cerebellar pathology were excluded from the study. The lobular volume was measured using the CERES (CEREbellum Segmentation) automated image processing protocol. Age classification was formed as 10 subgroups between 0 and 9 years old and 3 subgroups between 10 and 18 years of age. SPSS 22.0 was used for statistical analysis.

Results: In the study, it was observed that the total volume of the cerebellum and the volumes of the right and left cerebellar hemispheres increased significantly in the $0-1,1-2,2-3$ and 4-5 age groups. The most significant volumetric increase in all lobules of the cerebellum was detected in $0-1$ and 1-2 age groups. All lobules reached the highest average volume in the 10-12 age group. A statistically significant volumetric increase was detected in Lobules III, VI, VIIB and IX between 9 years and 10-12 years of age. The most significant difference with respect to gender was detected in age range $1-4(p<0.05)$.

Conclusion: Cerebellar diseases and degeneration often target specific areas of the cerebellum and are associated with specific symptom patterns. Obtaining cerebellum total and lobular volume data for pediatric age groups will provide important information. Cognitive task performance has been associated with the volumes in the lobules VI, VII, and IX in patients with cerebellar disease. It is known that activation patterns associated with leg and foot movements are localized to lobule III. In our study, it was found that the average volume value of these lobules in the $0-12$ age group increased regularly and was at the highest level at the age of 10-12 years.

Keywords: cerebellum, lobule, volume, anatomy, MRI 


\section{0-29}

Examination of the morphometric properties of the vessels waiting for the Willis polygon with diabetes and hypertension

Çiftçi R ${ }^{1}$, Çetin $A^{1}$, Ulubaba $\mathrm{H}^{2}$, Şenol D ${ }^{3}$, Özbağ $\mathrm{D}^{1}$

'Department of Anatomy, Faculty of Medicine, Inönü University, Malatya, Turkey; ${ }^{2}$ Department of Radiology, Inönü University, Turgut Özal Medical Center, Malatya, Turkey; ${ }^{3}$ Department of Anatomy, Düzce University, Faculty of Medicine, Düzce, Turkey

Objective: Pathological conditions such as hypertension, diabetes and such may affect the vessels in the Willis polygon (WP) negatively and cause important diseases such as stroke in the clinic. In this study, it was aimed to examine the morphometric properties of the vessels that make up WP in hypertensive and diabetic patients and to compare them with the vein measurements that make up WP in healthy individuals.

Methods: In our study, the CTA (Computed Tomographic Angiography) images of 168 (65 women, 103 men) individuals were measured retrospectively. The individuals included in the study were divided into four groups according to the diagnosis. Group $1(n=41)$ consisted of patients diagnosed with hypertension, Group $2(n=44)$ with diabetes, Group $3(n=43)$ with diabetes and hypertension, and Group $4(\mathrm{n}=44)$ healthy individuals. The diameters and lengths of the veins that make up the WP and the variations of these veins are included. In the study, a total of 16 diameter measurements from the right and left, and 10 segment length measurements were taken from the cerebral vessels.

Results: In our study, the mean age of women was $57 \pm 1.1$ years, and the average age of men was $59 \pm 0.7$ years. $86 \%$ of the individuals had adult configuration and $14 \%$ had fetal configuration. With fetal configuration $(27 \%)$, it was mostly seen in Group 2. The most common variation was aplasia (36\%). The percentage of aplasia is most common in Group 1 (43\%) and the most common vessel posterior communicating artery (AComP). Right from the WP, anterior cerebral artery (ACA) diameter of segment A1, right posterior cerebral artery (PCA) $\mathrm{P} 1$ and $\mathrm{P} 2$ segments diameters, right and left a. The diameters of the cerebri media (MCA) M1 segment differed in both men and women in all groups.

Conclusion: It was determined that the coexistence of diabetes and hypertension may cause greater damage to the cerebral vessels of the individuals compared to the other groups.

Keywords: CTA, Willis polygon, aplasia, hypertension, diabetes

\section{0-30}

A look at the future of anatomy education

Zeybek A, Özkan M

Department of Anatomy, Faculty of Medicine, Tekirdağ Namık Kemal University, Tekirdă̆, Turkey
Anatomy has been taught face to face and practically from the past to the present within the framework of gross anatomy training on cadavers. Technological innovations were added to anatomy education with the invention of X-ray in 1895 and the development of radiological imaging methods. Due to the Covid-19 pandemic, which emerged at the end of 2019 and has been affecting the world and our country as of March 2020, face-to-face training could not be carried out and the use of distance education methods was required. In this process, in addition to synchronous or asynchronous video lessons in the current digital learning environments, three-dimensional atlases, mobile applications and different digital platforms have also started to be used by students and faculty members. It can be easily predicted that distance anatomy education will continue in the future as an addition or support to face-to-face education, although it is not clear when the pandemic process will terminate. The proximity of today's students to digital applications in their learning process and the increasing number of medical faculty students in recent years are influential on our prediction. In this article, we will discuss that distance anatomy education will be required today and in the future and will cause a change in the learning and teaching habits of students and scholars.

Keywords: Covid-19, pandemic, anatomy education, distance education

\section{0-31}

Student thoughts on vertical integration of clinical anatomy education

$\underline{\text { Babacan } \mathrm{S}^{1}}$, Tuncel Çini $\mathrm{N}^{2}$, Kafa İ $\mathrm{M}^{2}$

'Department of Anatomy, Faculty of Medicine, Harran University, Şanlıurfa, Turkey; ${ }^{2}$ Department of Anatomy, Faculty of Medicine, Bursa Uludağ University, Bursa, Turkey

Objective: In medical education, which is in a continuous change and development, anatomy education is included in horizontal integration where similar subjects applied in preclinical basic sciences are given simultaneously by different disciplines, vertical integration where basic and clinical sciences are taught simultaneously, and spiral integration that includes both types of integration. The adaptation of the knowledge and skills acquired in pre-clinical medical education to the clinic by vertical integration will reveal the need for the acquired knowledge and will be more permanent when combined with clinical skills. The aim of the study was to determine the needs of the students in clinical internship education about anatomy and to plan the teaching of anatomy lessons related to the relevant subjects before clinical internships by rearranging the curriculum as needed.

Methods: The study was carried out on a questionnaire form applied to 4th, 5th and 6th grade students of Bursa Uludağ University Faculty of Medicine. In addition to demographic 
information, 16 multiple-choice and 6 open-ended evaluation questions were asked in the questionnaire.

Results: In the first phase of the study, 102 medical faculty students answered the questionnaire questions. $68.8 \%$ of the participant stated that they did not remember the anatomy lesson they took in pre-clinical basic education at the beginning of clinical internships; $82.4 \%$ of them stated that they needed the anatomy knowledge they got during their basic medical education during their clinical education and they need to study this information again; $86.2 \%$ of them stated that the systematic anatomy education given during the basic medical education period should definitely be given again at the beginning of the relevant internship within the clinical sciences.

Conclusion: In the Anatomy-Core Curriculum for Turkey Report in 2016, it was recommended to repeat anatomy and increase the details in clinical internships. As a result of the feedback received from the students with current study, it was seen that some of the anatomy information obtained during the pre-clinical basic sciences period was forgotten in the process until the clinical internship period and they should be remembered again. We believe that the implementation of the requirements of the suggestion, presented in the final report of Anatomy-Core Curriculum for Turkey Consensus in September of 2016, about integrated clinical anatomy lessons should be taken into consideration and implemented in clinical internships, which was accepted with a rate of $88 \%$.

Keywords: anatomy education, clinical anatomy, vertical integration, medical education

\section{0-32}

An evaluation on the anatomic studies published in the plastic surgery journals indexed in $\mathrm{SCl}$ and $\mathrm{SCI}-\mathrm{E}$

Cezairoğlu MA, Öztürk Köse Ö, Balcığlu HA

Department of Anatomy, Faculty of Medicine, Sağlık Bilimleri University, Istanbul, Turkey

Objective: This study was conducted to evaluate the anatomical studies published in plastic surgery journals indexed in Science Citation Index (SCI) and Science Citation IndexExpanded (SCI-e) in terms of number, ratio and content.

Methods: The journals published in the field of plastic surgery were accessed using the Web of Science database. All issues of these journals between January 2009 and May 2020 were scanned and anatomical studies published in these issues were selected. Anatomical studies were classified according to their profiles and summarized in the form of tables and graphics.

Results: Between January 2009 and May 2020, 17 journals in the field of plastic surgery within the scope of SCI and SCI-e were identified. The total number of anatomical studies published in these journals was 561 . When the articles in question were examined, it was observed that the studies were mainly cadaver studies (42\%). Cadaver studies were followed by clini- cal studies-surgical technique studies (27\%), reviews (11\%), retrospective studies $(8.5 \%)$, radiological anatomy studies $(6.5 \%)$ and case reports (4\%).

Conclusion: In the period from the beginning of 2009 to the first half of 2020, a general decrease was observed in the number of studies based on anatomical sciences published in plastic surgery journals. This decline may be an urgent signal that the space allocated to anatomy, which forms the basis of medical sciences, should be increased in both undergraduate and residency periods. One of the conclusions of this study is that anatomical case reports find their places in plastic surgery journals and are always clinically popular. Based on the determination that the number of anatomy-based studies in journals covered by SCI is much higher than in journals covered by SCI-e, it can be concluded that studies involving anatomical sciences may have a higher impact.

Keywords: anatomical sciences, plastic and aesthetic surgery, web of science

\section{0-33}

Determination of knowledge level of midwifery students about pelvis region anatomy

Özcan E ${ }^{1}$, Çetin $\mathrm{R}^{2}$, Karaca Oे $^{1}$

'Department of Anatomy, Faculty of Medicine, Balıkesir University, Balıkesir, Turkey; ${ }^{2}$ Department of Anatomy, Institute of Health Sciences, Balıkesir University, Balıkesir, Turkey

Objective: The aim of this study was to determine the knowledge level of the midwifery students studying at the Faculty of Health Sciences of Balıkesir University for pelvic region anatomy.

Methods: 294 (89.6\%) of 328 midwifery students participated in the study. All students participating in our research have completed their anatomy education. A questionnaire consisting of 37 questions, 6 of which were sociodemographic, 14 with pelvic region organs, 12 with pelvic region bones and 5 with pelvic region diameters were applied. The data were analyzed by using the SPSS (Statistical Package for Social Sciences) 22.0 package program.

Results: The mean age of the students was $20.71 \pm 1.88$ years (minimum: 18, maximum: 30 ). It was determined that $51 \%$ of the students were Anatolian high school, 33.7\% health vocational high school, $8.5 \%$ general high school, $1 \%$ vocational high school and $5.8 \%$ other high school graduates. The success rate of 14 problems related to the pelvic organs directed to the students was found to be $50.64 \%$. However, the success rate of 12 questions about the pelvic region bones was $55.96 \%$ and the success rate of the 5 problems related to pelvic region diameters was $27.56 \%$.

Conclusion: It was determined that the students who participated in our study had a moderate level of knowledge about the bones and organs related to the anatomy of the pelvic region in general. However, it was found that the information about the 
diameter of the pelvic region was very weak. It is thought that these students will contribute to strengthening their weak knowledge about pelvis region anatomy and being better midwives in their professions.

Keywords: anatomy, midwifery students, education, anatomy of pelvis

\section{0-34}

The effect of 2/4 finger length ratio on the anatomy exam success of midwifery students

\section{Özcan E}

Department of Anatomy, Faculty of Medicine, Balıkesir University, Balıkesir, Turkey

Objective: This study was conducted to evaluate the relationship between midwifery students' ratio of second and fourth finger lengths and their anatomy exam scores.

Methods: 294 (89.6\%) of a total of 328 midwifery students participated in our study. Students' right and left hand index (2P) and ring finger (4P) lengths were measured with a digital caliper. In our study, those with the 2 nd finger longer than the 4 th finger (2P / $4 \mathrm{P}$ ratio over 1 ) were defined as the estrogen dominant group. The ones whose 2 nd finger is shorter than the 4th finger ( $2 \mathrm{P} / 4 \mathrm{P}$ below 1$)$ were defined as the testosterone dominant group. The grades taken by the students in the anatomy course midterm and final exams were recorded in Microsoft Excel program. The collected data were analyzed and interpreted using the SPSS (Statistical Package for Social Sciences) 22.0 package program.

Results: Female students with an average age of $20.71 \pm 1.88$ years (minimum: 18, maximum: 30) participated in our study. The average of the students' midterm exam was $78.28 \pm 13.11$ in the first year, $71.36 \pm 18.89$ in the second year, $80.58 \pm 12.55$ in the third year and $76.86 \pm 13.01$ in the fourth year. The final averages were $68.28 \pm 13.11$ in the first year, $65.91 \pm 17.07$ in the second year, $69.70 \pm 10.15$ in the third year and $70.75 \pm 11.79$ in the fourth year. When the classes were compared with each other, the difference between the average of visas was found to be statistically significant $(\mathrm{p}<0.05)$, while there was no statistically significant difference between the final averages ( $p>0.05$ ). As a result of the correlation analysis, it was determined that there is a negative correlation between the right hand testosterone dominant group and the midterm exam grades $(r=-$ 0.229; p <0.05).

Conclusion: In our study, the increase in the testosterone level in the right hand causes the midterm grades of the anatomy lesson to decrease. Anatomy education is of great importance in raising qualified midwives. For this reason, we believe that midwifery students should be given a good anatomy education. Keywords: anatomy, 2/4 finger length ratio, education, anatomy education

\section{0-35}

Histological architecture of palatine tonsil and its association with deep tissue

Çetin $\mathrm{AZ}^{1}$, $\underline{\text { Adıgüzel E }}{ }^{1}$, Kara $\mathrm{CO}^{2}$, Bir F $^{3}$

'Department of Anatomy, Faculty of Medicine, Pamukkale University, Denizli, Turkey; '2Department of Otorhinolaryngology, Faculty of Medicine, Pamukkale University Denizli, Turkey; ${ }^{3}$ Department of Pathology, Faculty of Medicine, Pamukkale University, Denizli, Turkey

Objective: Clinically, the palatine tonsil is a lymphoid structure that mostly is subject to surgery. Complications occurring in surgical procedures are mostly related to the anatomical feature of palatine tonsil. The aim of this study is to evaluate the relation of palatine tonsil with deep tissue in the tonsillar fossa and to reveal the spatial distribution of the tissues in the lateral wall.

Methods: The research was carried out on palatine tonsil samples of thirty patients who underwent tonsillectomy for chronic tonsillitis or tonsillar hypertrophy within Pamukkale University Faculty of Medicine Ear Nose and Throat Department. The samples taken were subjected to histological examination and the relationship of tonsil tissue with pharynx tissue and distribution of adjacent tissues were revealed. In the three-dimensional evaluation of neighbouring tissues, consecutive serial sections were taken in accordance with the stereological principles, and measurements were made on these serial sections with the help of the Image J program. The adjacent surface of the palatine tonsil with the pharynx is divided into $9 \times 27$ units, and the tissue adjacent to each area is coded and graphed by finding the frequency and mean of the distributions.

Results: In this study, in which we analysed the palatine tonsil samples, $80 \%$ connective tissue, $15 \%$ seromucous gland tissue and $5 \%$ muscle tissue were detected on the lateral surface of palatine tonsil. In terms of the spatial distribution of these three tissues adjacent to the lateral face of the palatine tonsil, in the postero-superior of the seromucous gland tissue (29.14\%), in the posteromedial of the muscle tissue $(11.11 \%)$ and in the remaining areas connective tissue was more intense. The findings were statistically significant. $(\mathrm{p}<0.0001)$.

Conclusion: As a result, this study has revealed the relationship of palatine tonsil with pharynx tissue at histological level. According to the findings we obtained, there is no homogeneous tissue distribution adjacent to the pharynx tissue of palatine tonsil, and it shows different locations as salivary gland, muscle tissue and connective tissue. In terms of localization of these tissues, salivary gland is predominant in the posterosuperior part and muscle tissue is predominant in the posteromedial part. This feature can be a guide for planning surgical procedures in tonsillectomy surgeries. This work was supported by PAU Scientific Research Projects Coordination Unit (Project number: 2019SABE013).

Keywords: palatine tonsil, seromucous gland tissue, Weber's gland, connective tissue, muscle tissue 


\section{0-36}

Evaluation of nasolacrimal duct morphometry in Turkish population: a preliminary results

Vatansever A $^{1}$, Demiryürek D ${ }^{2}$, Erçakmak Güneş B ${ }^{2}$, Gümeler E ${ }^{3}$ 'Department of Anatomy, Faculty of Medicine, Balıkesir University, Balıkesir, Turkey; '2Department of Anatomy, Faculty of Medicine, Hacettepe University, Ankara, Turkey; ${ }^{3}$ Department of Radiology, Faculty of Medicine, Hacettepe University, Ankara, Turkey

Objective: Nasolacrimal duct, lies between lacrimal bone and maxilla, opens into inferior nasal meatus. Due to its canalshaped structure, obstructions of nasolacrimal canal are seen commonly. Numbers of studies have been published to evaluate nasolacrimal duct morphometry. However, anthropometric values could change between different populations. Therefore, a smaller number of studies have been published which evaluated the nasolacrimal duct morphometry regarding anthropometric diameters in different populations. Considering craniometric values during nasolacrimal duct surgeries has a significant importance for a successful surgery and high-quality postoperative period. Aim of this study was a detailed evaluation of nasolacrimal duct morphometry in Turkish population considering cranio-metric properties.

Methods: In our study, computed tomography image series of the head region of 23 female and 22 male participants, mean age 56.8 (27-82), have been included, retrospectively. Participants who had any pathology in the head region were excluded from the study. Antero-posterior diameter of cranium was measured using midsagittal sections of computed tomography images. Furthermore, antero-posterior and latero-lateral diameters of nasolacrimal duct at its starting point were recorded. Additionally, vertical angle of nasolacrimal duct and the distance to anterior surface of maxilla at the point of its opening into inferior nasal meatus were measured. All data was imported into SPSS v23 software to perform statistical analyses.

Results: Evaluated parameters did not have statistically significant changes with age. However, antero-posterior diameter of cranium (mean $17.76 \mathrm{~cm} ; 15.96$ to $19.56 \mathrm{~cm}$ ), latero-lateral diameter of the nasolacrimal canal at the starting point on the left side (mean $3.57 \mathrm{~mm} ; 1.99$ to $5.17 \mathrm{~mm}$ ) and the distance to anterior surface of maxilla at the point of its opening into inferior nasal meatus on the right side (mean $1.05 \mathrm{~cm}$; 0.66 to 3.57 $\mathrm{cm})$ were statistically longer in men than women $(\mathrm{p}<0.05)$.

Conclusion: Nasolacrimal duct obstructions, could be congenital or acquired, are complex pathologies which have various etiologies and prognosis and encountered frequently by ophthalmologists. There are surgical or non-surgical treatment methods for those pathologies. According to our preliminary results, most of the evaluated parameters did not demonstrate statistically significant differences between genders. These results could demonstrate that surgeons could decide their surgical techniques without considering gender differences.
Keywords: nasolacrimal duct, computed tomography, obstruction

\section{0-37}

Topography of the external branch of the superior laryngeal nerve and its importance in surgical interventions: cadaver study

Nteli Chatzioglou G, Sarı E, Gayretli Ö, Coşkun O, Öztürk A, Gürses İA

Department of Anatomy, Faculty of Medicine, Istanbul University, Istanbul, Turkey

Objective: Injury of the external branch of the superior laryngeal nerve (EbSLN) can cause a hoarse or weak voice due to the functional loss (dysergia) of cricothyroideus muscle. The purpose of the current study; to reveal the topography of EbSLN in Turkish population, to prevent injury to EbSLN during surgical intervention in the anterior neck region.

Methods: Landmarks were determined for the detection of the EbSLN. In total, 26 bilateral hemilarynges (4 females, 22 males) were dissected. The distance between the piercing point (PP) of the EbSLN, the superior pole of thyroid gland(ST) and the laryngeal prominence (LP) of thyroid cartilage were measured. All measurements were compared left and right.

Results: The distances from PP to ST were measured as $10.66 \pm 4.76 \mathrm{~mm}$ (transverse) and 2.9 $\pm 9.70 \mathrm{~mm}$ (vertical), from PP to LP $23.36 \pm 7.79$ (transverse) and $13.02 \pm 5.38$ (vertical), from ST to LP $10.5 \pm 6.59 \mathrm{~mm}$ (transverse) and $33.75 \pm 7.84$ (vertical). PP to LP was found $34.26 \pm 6.62 \mathrm{~mm}$ lateral and $10.49 \pm 7.68 \mathrm{~mm}$ inferior of the laryngeal prominence. While EbSLN ended in the cricothyroid muscle (CT) $50.01 \%$, it ended in $42.3 \%$ inferior constrictor pharyngeus (ICP) and $7.69 \%$ often by giving branches to the thyroid gland.

Conclusion: In this study, safe approaches for nerve protection during neck surgeries such as thyroidectomy with identified landmarks are described. In addition, it can be a safe and important guide in surgical approaches to be applied in the anterior neck region.

Keywords: superior laryngeal nerve, safe surgical approach, thyroidectomy, cadaver

\section{0-38}

Sex determination using frontal sinus diameters on direct radiography

Emekli E

Department of Radiology, Etimesgut Şehit Sait Ertürk State Hospital, Ankara, Turkey

Objective: It may be necessary to identify skeletons that cannot be recognized for any reason. In cases where descriptive features such as DNA and fingerprints cannot be used, radiological 
examinations play an important role. Frontal sinus (FS) is absent at the beginning of life unlike other sinuses. Since its shape and developmental stages differ in each individual, it can be used in determining the sex and identity of individuals. In our study, we aimed to investigate the use of FS diameters in sex determination in anterior-posterio (AP) cranial radiographs.

Methods: 350 patients between the ages of 20-55 who have had AP skull radiography were included in the study. FS was classified as symmetrical, asymmetrical (right or left dominant), unilateral or bilateral aplasia. The longest diameters on both sides were divided into each other to evaluate the right and left asymmetry. Right height, right width, left height, left width measurements were made, the largest diameters were taken in the measurement. Independent t-test was used to compare diameter measurements between sexes. For the use of frontal sinus parameters in sex determination discriminate analysis method was used.

Results: The mean age of the patients was $31.23 \pm 11.9$ years in males, $34.45 \pm 10.75$ years in females, and $32.8 \pm 11.45$ years in both groups. Bilateral aplasia (12 men, 8 women) was detected in 20 patients, right aplasia in 19 patients ( 10 men, 9 women $)$, and left aplasia in 11 patients ( 7 men, 4 women). FS was symmetrical in 206 of the remaining 300 patients, right dominance in 33 patients and left dominance in 61 left patients. Measurements were made in the remaining 300 patients (154 men, 146 women). Right height, right width, left height and left width values were all statistically higher in males than females $(\mathrm{p}<0.05)$. In the analysis made for gender determination, it was determined that $71.2 \%$ of women and $68.2 \%$ of men were able to classify correctly. Correct classification for both sexes was calculated as $69.7 \%$.

Conclusion: We found that FS diameters were higher in men than in women and direct radiography could be used in sex determination at a rate of $69.7 \%$. According to this information, FS diameters can be used in forensic sciences in determination of sex and person, even if not alone.

Keywords: paranasal sinus, direct radiography, frontal sinus, sex determination

\section{0-39}

Transverse foramen as a guide during cervical spine surgery

Cetinok $\mathrm{H}^{1-3}$, Downie $\mathrm{SA}^{2}$, Cömert $\mathrm{A}^{4}$

'Department of Anatomy, Cerrahpaşa Faculty of Medicine, Istanbul University-Cerrahpaşa, Istanbul, Turkey; ${ }^{2}$ Department of Anatomy and Structural Biology, Albert Einstein College of Medicine, Yeshiva University, New York, USA; ${ }^{3}$ Department of Anatomy, Faculty of Medicine, Istanbul Atlas University, Istanbul, Turkey; ${ }^{4}$ Department of Anatomy, School of Medicine, Ankara University, Ankara, Turkey

Objective: In cervical vertebra surgeries, particularly reducing complications of vertebral artery (VA) injury; anatomy of VA and structures in its critical neighborhood is important. Lateral dissection, especially anterior cervical surgeries, is the greatest risk for VA; reference points should be determined accordingly. Aim of the study is to contribute to reduction of VA injury and complications in cervical region surgeries with the data obtained as a result of morphological, morphometric evaluation of transverse foramen (TF) through which the VA passes.

Methods: In Albert Einstein College of Medicine Anatomy Laboratories, set of 80 dry bones, C3, C4, C5, C6, C7 vertebra on both right (R) and left (L) sides; AP (anteroposterior), transverse diameters of TFs were measured with digital calipers. Each vertebra was evaluated in terms of morphological, variation, osteodegenerative changes. Measurements were evaluated statistically with the SPSS 11.5 Windows program. Average, standard deviation values were thus calculated.

Results: It was observed that TFs in different levels were double, as of the number ' 8 ', two foramen side by side joined with a thin bony tissue in the middle (double, accessory / split). 49 of C6 vertebra were examined both side (98 TF), 18 of them on $\mathrm{R}, 20$ of them on L, for total of 38, 52 of C5 vertebrae (104 TF), 18 on R, 14 on L; for total of 32, 52 of C4 vertebrae (104 TF), 6 on R\& 5 on L, 11 of them were paired, these were detected as accessory or split TF. Spur formations in which uncus heterotrophically extended over the TF were observed in 1-C3, 2-C4, 1-C5, 2-C6 vertebrae. When TF in 2 different sets were compared in terms of their diameters, it was observed that they were differentiated on both sides. Osteophytes were detected in 1-C5 vertebra from the corpus vertebrae towards the TF. When the boundaries of 1-C6 TF were examined, it was observed that one side was in the form of a sharp edge instead of a circular contour. In our study, it was observed that TF diameters on both sides increased from C3-C6. (R: $4.95 \pm 0.62$ to $5.49 \pm 1.01 \mathrm{~mm}$, L: $5.10 \pm 0.56$ to $5.86 \pm 1.21 \mathrm{~mm}$ )

Conclusion: Increasing TF diameters from C3-C6 level, variations in TF (double, incomplete formations etc.) affect the course of VA, were especially risky in anterior approaches. Preoperative imaging, evaluating anatomy of the region, the osteodegenerative changes of VA (structures in the vicinity of TF), will contribute to reducing postoperative complications of VA injury in cervical region surgeries.

Keywords: transverse foramen, cervical spine, cervical vertebra, clinic anatomy, vertebral artery, variation

\section{0-40}

\section{Determination of the os trigonum prevalence with radiographic images in Alanya population}

\section{Candan B, Dikici R}

Department of Anatomy, Faculty of Medicine, Alanya Alaaddin Keykubat University, Antalya, Turkey

Objective: Os trigonum is one of the most common accessory ossicles in the ankle region and it is usually detected as an inci- 
dental radiological finding. It is located in the posterolateral aspect of the talus and rarely, maybe bipartite. Os trigonum can cause chronic ankle pain in sportive activities that require forced plantar flexion. The prevalence of the os trigonum varies between $1-25 \%$. The purpose of this study is to determine the morphology and prevalence of os trigonum by retrospectively examining the radiographic images of the patients who applied to the hospital with complaints of ankle pain in Alanya.

Methods: In this study, foot and ankle radiograph images from anteroposterior and lateral angles of 400 patients, between the ages of 20-80 with no external pathology and anomaly were examined. Before the study, approval was obtained from the Medical Faculty Clinical Research Ethics Committee (ALKÜKAEK). The distribution by gender of the os trigonum, prevalence, and bilateral presence of the os trigonum was evaluated. Measurement and evaluation were carried out by a single researcher. In the presence of the os trigonum its length and width were measured. The mean and standard deviation (SD) values of these measurements were calculated.

Results: We found that 17 of 400 patients (4.25\%) had os trigonum in their ankles. These bones were seen in 7 female patients and 10 male patients. We observed that os trigonum was found bilaterally in one female patient.

Conclusion: In this study, it was aimed to determine the morphology and prevalence of os trigonum by retrospectively examining the radiographic images of the patients who applied to the hospital with the complaint of the ankle pain in the Alanya population. The incidence of os trigonum was determined as $4.25 \%$ in our study. Confusion of common accessory bones with avulsion fractures may result in unnecessary treatment attempts. Therefore, the frequency and localization of accessory bones should be well known. We hope that the prevalence of the os trigonum which we determined as a result of our study can be useful as it draws attention to not being confused with avulsion fractures, which are common on radiographs.

Keywords: os trigonum, accessory ossicles, ankle, radiography, anatomy

\section{0-41}

Could the incidence and distribution of os supratrochleare dorsale and patella cubiti be the key to correct diagnosis?

Aksu F, Akkoç RF, Aydın S

Department of Anatomy, School of Medicine, Firat University, Elazığ, Turkey

Objective: Patella cubiti is a rare sesamoid bone within the musculus triceps brachii tendon. Os supratrochleare dorsale is another rare sesamoid bone that can be found in the anterior of the musculus triceps brachii tendon or in the fossa olecrani.
Both sesamoid bones are usually asymptomatic. However, it may rarely cause clinical limitation of the extension of the elbow joint or elbow joint pain. The aim of this study is to determine the incidence of patella cubiti and os supratrochleare dorsale and the differences between genders.

Methods: This study was approved by the Non-Invasive Investigations Ethics Committee of the University of Firat (meeting date 29.04.2020; meeting issue 2020/07-16). In the study, direct radiographs of 1646 people, 959 female and 687 male who applied to Frrat University Hospital between 01.01.2016 and 31.01.2019 and had elbow joint radiographs, were evaluated retrospectively. Of the radiographs evaluated, 346 were right and left elbow radiographs of the same person, 689 were just right elbow radiographs, and 611 were only left elbow radiographs.

Results: The incidence of os supratrochleare dorsale was 20 $(2.08 \%)$ in 959 women, 18 (2.62\%) in 687 men, 38 (2.3\%) in 1646 in total. The prevalence of patella cubiti was found to be $4(0.42 \%)$ in 959 women, $9(1.31 \%)$ in 687 men, $13(0.79 \%)$ in 1646 in total. In addition, the frequency of incidence of only the right elbow, only the left elbow, and both sesamoid bones in both elbows was determined by making the distinction between men and women and without gender discrimination.

Conclusion: We think that knowing the incidence and distribution of these sesamoid bones will help patients with complaints such as limiting elbow extension and pain in the elbow area will help to make an early and accurate diagnosis.

Keywords: os supratrochleare dorsale, patella cubiti, elbow joint, direct graphy, sesamoid bone

\section{0-42}

\section{Investigation of foot accessory bones with computed tomography}

Aslantürk $^{1,2}$, Özbağ D $^{2}$

'Malatya Education and Research Hospital, Malatya, Turkey; ${ }^{2}$ Department of Anatomy, Health Science Institute, Inönü University, Malatya, Turkey

Objective: Accessory ossicles of foot usually occur due to fusion defect of adjacent bone. In literature, the accessory bones are investigated via cadavers or radiographic images in many studies. In this study, we aimed to investigate accessory bones of foot via computed tomography (CT) images.

Methods: We retrospectively investigated the CT images between January 2019-January 2020. Patients older than 18 years old and with CT images including foot and ankle were included in the study. Age, sex, side and existing accessory bones were recorded.

Results: Two hundred and forty CT images included in the study. One hundred and sixty-five patients were male and 75 were female. There were 40 left, 41 right sides and 159 bilater- 
al foot CT images. Mean age of patients were 36.9 (range 18-65 years) years. There was no accessory bone in 165 $(41.3 \%)$ feet. Most common accessory bone was os naviculare (127 feet, $31.8 \%$ ). There were 32 type I os naviculare, 47 type II and 48 type III. There were os trigonum in 54 feet (13.5\%), os peroneum in 51 feet $(12.7 \%)$, os intermetatarseum in 25 feet (6.2\%) and os calcanei secundarius in 6 feet $(1.5 \%)$.

Conclusion: In our study, we detected a higher rate of accessory bone when compared with previous studies from our country. Our higher rate (58.6\%) from previous studies $(18.3 \%$ and $21.2 \%$ ) may be related to higher sensitivity of CT to detect bony structures compared to conventional radiographies. In addition, in our study, unlike previous studies, it was found that the incidences of os peroneum and os trigonum were almost similar.

Keywords: prevalence, tomography, accessory bone, foot, radiological

\section{0-43}

Acromion types and morphometric evaluation of shoulder joint by magnetic resonance imaging

$\underline{\text { Koca R }}{ }^{1}$, Fazlıŏulları Z2, Aydın BK ${ }^{3}$, Durmaz $\mathrm{MS}^{4}$, Karabulut $\mathrm{AK}^{2}$, Ünver Doğan $\mathrm{N}^{2}$

'Department of Physiotherapy and Rehabilitation, Afyonkarahisar University of Health Sciences, Faculty of Health Science, Afyonkarahisar, Turkey; 'Department of Anatomy, Medical Faculty, Selçuk University, Konya, Turkey; ${ }^{3}$ Department of Orthopedics and Traumatology, Medical Faculty, Selçuk University Konya, Turkey; 'Department of Radiology, Medical Faculty, Selçuk University, Konya, Turkey

Objective: To emphasize the importance of acromion types, presence of os acromiale and acromial morphometric measurements in the etiology, diagnosis and treatment methods of shoulder pain.

Methods: Our study was conducted retrospectively to the Department of Orthopedics and Traumatology, Faculty of Medicine, Selçuk University between 2010 and 2019, with some shoulder pain complaints such as subacromial impingement syndrome and rotator cuff tears, magnetic resonance imaging and no pathological problems, 100 of both sexes performed using images of the patient. 4 (four) patients who had undergone shoulder surgery for any reason, had tumor-like formations in the area or had trauma were excluded from the study.

Results: While type IV acromion was not encountered in our study, the most common type is type II acromion in both women (39 people) and men (23 people). Of the total acromions, $21 \%$ was type I, $62 \%$ was type II, and $17 \%$ was type III. There was statistically significant difference when the acromion slope was examined by types, but no significant difference was found when analyzed by gender. When the acromion length, coracoid process length and the distance between acromion and coracoid process were examined according to the types, no significant difference was found ( $>0.05)$, when analyzed by gender, it was observed that the lengths of acromion and coracoid process were significantly longer in males $(\mathrm{p}<0.05)$, but no statistically significant difference was found in the distance between acromion-processus coracoideus ( $>0.05)$. According to acromion types and gender; when the lateral acromial angle, acromial index and critical shoulder angle were compared, no statistically significant difference was found $(\mathrm{p}>0.05)$. There was negative correlation between lateral acromial angle and acromial index, critical shoulder angle but positive correlation between acromial index and critical shoulder angle. There was also a negative correlation between acromion slope and acromiohumeral distance. When we compared the acromiohumeral distance by gender, it was statistically significantly higher in males $(\mathrm{p}<0.05)$ and when we compared by types, there was no significant difference ( $>0.05)$. When we look at the distribution of os acromiale, a total of 5 were observed, 3 in women and 2 in men, and no statistically significant difference was found according to gender.

Conclusion: We think that our study will contribute to the current literature knowledge, shoulder joint morphometric measurements will contribute to the field of orthopedics clinically, and create an idea in distinguishing the problems that may occur according to gender and acromion types.

Keywords: acromion, magnetic resonance imaging, shoulder joint, subacromial impingement syndrome

\section{0-44}

\section{Comparison and demographic evaluation of ulnar} variance measurement methods

Öztürk S ${ }^{1}$, Kahraman $\mathrm{F}^{2}$, Salim $\mathrm{H}^{1}$, Acar B ${ }^{3}$, Çalgüner $\mathrm{E}^{4}$, Yolcular B ${ }^{5}$, Sindel $\mathrm{T}^{2}$, Sindel $\mathrm{M}^{1}$

'Department of Anatomy, Faculty of Medicine, Akdeniz University, Antalya, Turkey; 'Department of Radiology, Faculty of Medicine, Akdeniz University, Antalya, Turkey; ${ }^{3}$ Department of Orthopedics and Traumatology University of Health Sciences Antalya Education and Research Hospital, Antalya, Turkey; ${ }^{4}$ Department of Anatomy, Faculty of Medicine, University of Kyrenia, Kyrenia, Cyprus; ${ }^{5}$ Departments of Biostatistics and Medical Informatics Faculty of Medicine, Akdeniz University, Antalya, Turkey

Objective: The relative length difference between the radial and ulnar distal joint faces is defined as ulnar variance (UV). It is evaluated as neutral UV (both joint surfaces are at the same level), positive UV (ulnar joint face is more distal), and negative UV (ulnar joint face is more proximal). The relative length of the ulna compared to the radius or UV is seen as an important element in wrist pathologies. This length can be affected by age, genetic factors, load and elbow pathology. The aim of our study was to compare the measurement methods of ulnar variance and to provide information about whether the ulnar variance changed based on the demographic characteristics of patients. 
Methods: The ulnar variance was measured on wrist radiographs of a total of 124 patients (62 males, 62 females; mean age 48.5 years; range, 18 to 79 years) who were admitted to Akdeniz University Radiology Department between 01.01.2014 and 01.04.2019. Two methods, perpendicular and circular, were used to calculate the measurements. To determine ulnar variance on radiographs, the accepted standard view was used, which is a posteroanterior projection from the wrist in neutral forearm rotation, the elbow bent $90^{\circ}$ and the shoulder $90^{\circ}$ missed.

Results: The mean UV was 0.33 (range -4.3 to 5.7 ) $\mathrm{mm}$ in the measurements using the perpendicular method for both wrists of the patients. The mean UV was found to be 0.034 (range -5 to 5.7$) \mathrm{mm}$ in the circular method measurements. A statistically significant difference was found between the two measurement methods $(\mathrm{p}<0.001)$. In addition, no statistically significant difference was found between the UV measurements of the right and left wrist radiographs $(\mathrm{p}=0.662)$ using the perpendicular method and the UV measurements of the right and left wrist radiographs using the circular method $(\mathrm{p}=0.719)$. No statistically significant difference was found in the perpendicular and circulatory methods on the right side according to sex ( $\mathrm{p}=0.524$ and $\mathrm{p}=0.435$, respectively). However, in both methods, UV values were significantly different between the sexes in the left-hand measurements ( $\mathrm{p}=0.043, \mathrm{p}=0.047$ ).

Conclusion: We believe that the potential deficiency of the techniques in the literature, particularly in the circular method, arises from the differences in the concavity of the lunate fossa. We also consider that the pronosupination of the patient in the gravitational position affects the measurements. However, further large-scale, prospective studies are needed to confirm these findings.

Keywords: ulnar variance, age, aging, Kienbock's disease, ulna

\section{0-45}

\section{Cadaver embalming process during pandemic:} Zonguldak Bülent Ecevit University experience

Yilmazer Kayatekin AZ, Atalar K, Özer CM

Department of Anatomy, Faculty of Medicine, Zonguldak Bülent Ecevit University, Zonguldak, Turkey

Objective: Coronavirus 19 (Covid-19) outbreak has also affected cadaver dissection and vivisection, which are indispensable elements of anatomy education. Anatomy training will be conducted virtually until an unknown date. It is also risky to accept cadaver donation in cases where the suspicion of infection cannot be resolved. In this study, it is aimed to share the experiences we gained as a result of cadaver donation under pandemic conditions.

Case: Our cadaver reception process started with the application of the relatives of a 77-year-old male patient who died of trauma for cadaver donation. A report was prepared in accor- dance with the Turkey Anatomy and Clinical Anatomy Association instructions, Record of Receiving the Funeral (form no. 11) and the corpse was received. The corpse was kept in the Morgue of the Zonguldak Bülent Ecevit University (ZBEU) Medical Faculty Hospital until embalming preparations were completed. We consulted with the physicians who were responsible for the treatment of the patient at the ZBEU Medical Faculty Hospital. We learned that the patient didn't have any symptoms that would cause suspicion of infection or Covid-19 during the period of hospitalization and operation, so no testing was performed. Post-mortem Covid-19 tests couldn't be performed also. Personal protective equipment was provided before cadaver embalming. The prepared embalming solution was started to be given by using a serum set by effect of gravity. After 24 hours of fluid loading, there was a day break. The remainder of the fluid was also loaded in the second 24 hour session. Towards the end of the second session, blisters were observed on the extremities of the cadaver. We observed that the number of blisters did not change and they did not grow after the embalming was completed. Since they occurred towards the end of the embalming process, it was concluded that the blisters did not occur due to infection, but due to excessive loading of embalming fluid and post-mortem epidermolysis. After the embalming procedure, the laboratory was cleaned and ventilated. The tools used were disinfected. Waste was properly disposed of.

Conclusion: Cadaver is an indispensable element of anatomy education. Post mortem Covid-19 transmission has not yet been reported in the literature. Precautions to be taken against infections in laboratory conditions are always valid. Due to cadaver donation is very rare in our country, after the necessary precautions are taken, we think that it is possible to accept cadaver donations and perform embalming.

Keywords: cadaver, embalming, pandemic, Covid-19

\section{0-46}

History of the premaxillary bone: os Goethe or os Avicenna?

Dursun $\mathrm{A}^{1}$, İnce $\mathrm{F}^{2}$

${ }^{1}$ Department of Anatomy, School of Medicine, Süleyman Demirel University, Isparta, Turkey; ${ }^{2}$ Department of History of Medicine and Ethics, School of Medicine, Süleyman Demirel University, Isparta, Turkey

Objective: To investigate the premaxillary bone in The Canon of Medicine by Avicenna (980-1037), who is one of the pioneers of medicine.

Methods: The chapters on anatomy in Canon have been examined from the Turkish translation of Esin Kâhya and the English translation by Laleh Bakhtiar. National and international publications examining the premaxillary bone were evaluated.

Results: The Canon is a work that consists of five books and started to be written in 1012-1013. Avicenna explained com- 
prehensive general information about human anatomy in the first book of his work. The subject of the upper jaw is encountered in the fourth chapter, which is located in the first lower part of the fifth part of the book. Avicenna described the suture between the maxillas. In modern anatomy, the suture is called the median palatine suture. Avicenna also wrote that there are second and third sutures extending from the median palatine suture between the incisors and canines. In addition to these, he reported that there were two triangular-shaped bones between the aforementioned sutures and a fourth suture between the front two incisors. Although this information is not named in the Canon, it is understood that it is a description of a pair of bones located close to the midline in front of the maxilla, also known as "os incisivum", "os intermaxilla”, "os premaxilla" in modern anatomy. The first definition of the incisor bone in publications was made by Galen (129-200) in animals. Andreas Vesalius (1515-1564) wrote that this bone could not be found in humans. Volcher Coiter (1534-1576), Félix Vicq-d'Azyr (1748-1794), Johann Wolfgang von Goethe (1749-1832), and Pierre Marie Auguste Broussonet (1761-1807) are listed regarding the discovery of incisive bone in humans. However, the discovery is commonly attributed to von Goethe in the literature, this bone is also called "os Goethe".

Conclusion: It is understood that the premaxillary bone in humans was described for the first time in the Canon, although it is not specified by name. In the description, which is very close to today's information, it is explained that these bones are separate in the upper jaw. These are very striking in that they show the anatomy knowledge that Avicenna possessed ten centuries ago and conveyed to us with his work. Considering the chronological complexity in naming the premaxillary bone, we think that it is important to call the incisive bone "os Avicenna" and to mention the name Avicenna as it deserves in new studies.

Keywords: Avicenna, von Goethe, history, premaxillary bone, incisive bone

\section{0-47}

The contribution of scholars to the anatomy of the eye and adnexa in Islamic geography

Çınaroğlu $\mathrm{S}^{1}$, Zor $\mathrm{KR}^{2}$, Küçük E ${ }^{2}$

'Department of Anatomy, Faculty of Medicine, Niğde Ömer Halisdemir University, Niğde, Turkey; ${ }^{2}$ Department of Ophthalmology, Faculty of Medicine, Niğde Ömer Halisdemir University, Niğde, Turkey

Objective: With this study, we aimed to present the contributions of Islamic scholars on the anatomy of the eye and adnexa.

Methods: Full text articles in Turkish and English on the subject in "Google Scholar", "Pubmed", "Science Direct", "Web of Science" and "ULAKBIM" databases were scanned and analyzed.
Results: From the early period of Islam, muslim scholars have translated the ancient Greek medical works, and they reached a much more advanced level. Blindness was a major cause of disability in all Islamic geography, so physicians from islamic territory, particularly Yuhanna ibn Masawayh, Hunain Ibn Ishaq, Rhazes, Ali bin Isa, Ibn-i Sina, Ibn Al-Haitam, were particularly focused on the anatomy of eye and diagnosis and treatment of eye diseases. They reached an advanced level, leading Western European scientists in the following centuries.

Conclusion: Islamic scholars, benefiting from the works of other civilizations, made significant contributions to the anatomy of the eye by making human dissection, although it was prohibited.

Keywords: eye anatomy, eye diseases, islamic territory, blindness

\section{$0-48$}

\section{A women anatomist: Anna Morandi Manzolini \\ Özdemir B, Şanlı O, Dursun A \\ Department of Anatomy, Faculty of Medicine, Süleyman Demirel University, Isparta, Turkey}

It is surprising that one of the few women famous for their medical expertise before the modern age was not a midwife or a herbalist but an anatomist. In 18th century Bologna, Italy, the innovative teaching of anatomy by one of the female scientists Anna Morandi Manzolini (1716-74) with human samples and wax models is quite impressive. Without mentioning Morandi, the influence of women on the development of anatomy cannot be understood. Therefore, it is necessary to clarify the life and achievements of Morandi. Morandi grew up in a traditional home where marriage, children, and domestic lifestyle are natural choices for women. She received professional training as an artist in the leading local studios of Francesco Monti (1685-1768). She worked for 3 years on the production of anatomical wax models for the newly established Anatomy Museum at the University of Bologna in 1743. Morandi learned dissection by making extensive readings on anatomical texts. Morandi's status is complicated by her concurrent role as an anatomist and artist. She is one of the leading creators of accurate wax patterns of tissues and organs. Morandi's waxes bridge the gap between practical visual and theoretical text by combining fine arts and surgery to vividly reveal and theorize the functioning of the human body. She has the privilege of being the first to show small body parts, including capillaries and nerves, in wax. She was surprising because of her scientific knowledge during her public dissections, the beauty and anatomical accuracy of the wax models are internationally acclaimed and honored by numerous presidents. She has been appointed by the University of Bologna as the Public Modeler and Demonstrator of Anatomy and has been authorized to inspect more than a thousand cadavers delivered from public hospitals. She has special focus on the anatomy and physiology 
of all sensory organs, physical stimulation, nervous and motor effect. In her examples, she emphasized the functional details of vascularization and innervation in different parts of the human body. The collections describing the dynamic structures of the human body have served the education of medical students, the use by medical practitioners and experts. Morandi set a new purpose for the wax model, presenting it as a teaching tool for correct anatomy. She organized the wax models as a 250 -page notebook based on gradual dissection with a systematic approach. The effects of this notebook on the development of contemporary anatomical studies are enormous.

Keywords: women anatomist, beeswax model, anatomy education, dissection

\section{0-49}

\section{Anatomic theater from past to present}

Yilmaz V, Yazan H, Dursun A

Department of Anatomy, Faculty of Medicine, Süleyman Demirel University, Isparta, Turkey

The anatomical theater is an amphitheater shaped hall used for anatomy education in early modern universities, with a table in the center for human body dissections. Although the dissections were usually carried out for the purpose of education for medical students and scientists, they were performed in the atmosphere of a fun and interesting theater play for the public and gave a ceremonial atmosphere that proved the greatness of God. The concept of anatomical theater originated during the Renaissance by Alessandro Benedetti (1450-1512), professor of Anatomy and Surgery at the University of Padova. The first temporary anatomical theater was built by Alessandro Benedetti. Alessandro Benedetti emphasized the importance of observation in medical education with his words "we see the truth in the theater". Padova Anatomical Theater, the first permanent anatomical theater, was built in 1594 by Professor Hieronymus Fabricius Ab Aquapendente (1537-1619) in Padova. This theater was a concentric, conical structure with six rows from low to high, capable of accommodating 300 people, positioned no more than 10 meters from the dissection table, and illuminated by candle chandeliers held by students, without windows. In the first place were the anatomy professors, rectors, city councilors, medical faculty members and representatives of the Venetian nobility, the second and third rows were students, and the other rows were the audience. Today's anatomy theaters are in Italy; Padua anatomical theater (1594), Archiginnasio Anatomical Theater (1637), Giovanni Tumiati Anatomical Theater (1731), Modena Anatomical theater (1775), Modica anatomical theater built in the 17th century, Anatomical theater established in the Ancient San Gallicano Hospital (1826), Pistoia Ospedale Del Ceppo Anatomical Theater founded in the late 18th century Aula Scarpa Anatomical Theater, Netherlands; Leiden University anatomical theater (1596), Waag anatomical theater (1691), in Spain;
Sala Gimbernat Anatomical Theater, Gran Anatomic theater, Russia; Kunstkamera Anatomical Theater (1706), the anatomical theater in the St. Thomas church, in Belgium; Leuven Anatomical theater (1743). The only anatomical theater in a region outside of Europe is the Kazan University anatomical theater, founded in 1837 . Today, most of these anatomical theaters are used as museums and some are still used as conference halls and libraries. The development of anatomical theater has a central place in the history of medical education. The aim of the theater is to "see" and "show", and represents a major change from dissections in the homes of anatomists for the benefit of small groups of students to dissection performances open to the public. Moreover, anatomical theater brought along a systemic and methodological revolution in medical education, and enabled the medical curriculum to be renewed and developed. This practical medical training allowed integration and partnership between teachers and students. Thus, by removing communication barriers, it promoted individual participation towards optimal use of materials for active learning.

Keywords: theater, history, renaissance, dissection

\section{0-50}

Cadaveric case report: a rare variation between piriformis muscle and sciatic nerve

Cimen K, Doğruyol G, Otă̆ İ, Çimen M

Department of Anatomy, Faculty of Medicine, Sivas Cumhuriyet University, Sivas, Turkey

Objective: During routine dissections encountered the variational structure between the piriformis muscle and sciatic nerve and its clinical connection was investigated.

Case: During the dissections in the male cadaver on the right extremity unilaterally: It was determined that the tibial nerve and common fibular nerve exit together from the foramen infrapiriforme as a normal, but variationally on the nerves routes were separated by the piriformis muscle. The tibial nerve passes under the piriformis muscle, and the common fibular nerve passes above the muscle. No difference was observed in other structures using foramen suprapiriforme and infrapiriforme.

Conclusion: It was concluded with literature investigations that the variation encountered was rare with a rate of $3.3 \%$. Also, it has been determined that these differences seen between muscle and nerve structures usually cause entrapment of the sciatic nerve and are clinically seen as the cause of piriformis syndrome. We recommended that paying attention to this variational structure, may be useful for clinicians about piriformis syndrome treatments.

Keywords: piriformis muscle, sciatic nerve, piriformis syndrome 


\section{0-51}

Extraordinary duplication of cystic duct: a cadaveric case study

Akbulut $\mathrm{Y}^{1}$, Karakas Tanır $\mathrm{AB}^{1}$, Uzuner $\mathrm{MB}^{1}$, Boduç $\mathrm{E}^{1}$, Sertçelik $M^{2}$

'Department of Anatomy, Faculty of Medicine, Kafkas University, Kars, Turkey; ' ${ }^{2}$ Department of Chemical Technologies, Faculty of Engineering and Architecture, Kafkas University, Kars, Turkey

Objective: The objective of this study is to describe the unusual anatomical variation of cystic duct in extrahepatic bile ducts.

Case: This unusual duplication of cystic duct was determined in a white adult female cadaver fixed with formalin. Anatomical structures were photographed and transferred to the personal computer. The lengths of the structures were measured with the ImageJ software (version 1.53a), which is a photogrammetric measurement method. The weights of stones from gallbladder were measured with electronic precision balance (accuracy $0.0001 \mathrm{mg}$, Type AX120, Shimadzu, Japan). During the routine dissection performed to reveal the biliary system of the cadaver, it was determined that two ductus cysticus with diameters of $4.426 \mathrm{~mm}$ and $3.233 \mathrm{~mm}$ were opened to the vesica biliaris, which is $112.812 \mathrm{~mm}$ in length and $67.315 \mathrm{~mm}$ in width, respectively. It was observed that the cystic duct with a small diameter was in the "Y" form, continued $22.445 \mathrm{~mm}$ after separating from the common hepatic duct, and then divided into two ducts with diameters of $3.103 \mathrm{~mm}$ and $3.274 \mathrm{~mm}$. Ducts entered the gallbladder through two separate openings. The diameter of the thick and long duct was measured $4.426 \mathrm{~mm}$, length was $26.055 \mathrm{~mm}$ and the diameter of the thin and short duct was measured as $0.882 \mathrm{~mm}$, length as 7.502 $\mathrm{mm}$. Common hepatic duct was found to have a diameter of 8.667 $\mathrm{mm}$ and a length of $39.875 \mathrm{~mm}$. It was observed that the content of gallbladder was like mud. A large walnut-sized gallstone weighing $12.624 \mathrm{mg}$ was detected in gallbladder, together with 7 irregularly shaped stones ranging in weight from $26-255 \mathrm{mg}$. As a result of powder XRD diffraction analysis of gallstones, some characteristic diffraction peaks were determined as $33.59,17.02$, $6.87,5.95,4.90$ and 3.83 at $2{ }^{\circ}$. Due to the diffractogram results matching up with the diffractogram of cholesterol, it was defined that the stones were composed of cholesterol.

Conclusion: Diagnosis of these anatomic variations especially in cholecystectomy prior to surgical procedures may prevent iatrogenic injury to bile ducts, bile leakage, fistula, and cystic artery injury.

Keywords: anatomical variation, cystic duct, extrahepatic bile ducts, gallstone

\section{0-52}

\section{Double aortic arch: case report}

Etli $M^{1}$, Avnioğlu $S^{2}$, Dikici R ${ }^{2}$

'Department of Cardiovascular Surgery, Faculty of Medicine, Alanya Alaaddin Keykubat University, Antalya, Turkey; 'Department of Anatomy, Faculty of Medicine, Alanya Alaaddin Keykubat University, Antalya, Turkey
Objective: Double aortic arch (DAA) is defined as a type of vascular ring malformation. Its incidence is less than $1 \%$ of congenital heart disease. The Congenital DAA which accounts for $46-76 \%$ of the complete rings is the most common vascular malformation in the congenital rings. We wanted to add to the literature a case of DAA, a rare congenital vascular malformation that can be easily misdiagnosed.

Case: A 51-year-old male patient was admitted to the Chest Diseases Polyclinic with complaints of chest pain and stinging in breathing. Computed tomography (CT) showed that the patient had a double aortic arch and the patient was referred to the Cardiovascular Surgery Polyclinic. In the echocardiography (ECHO) of the patient, signs of cardiac hypertrophy were observed but there were no signs of heart failure. Electrocardiography (ECG) was in sinus rhythm, there was no evidence of hypertension, and blood values were normal. The diameter of the right aortic arch was $22.2 \mathrm{~mm}$, and the diameter of the left arch aorta was $14.5 \mathrm{~mm}$. Ascending aorta diameter was $30 \mathrm{~mm}$. The patient had no specific complaints and no medical or surgical treatment was recommended because his physical examination was normal.

Conclusion: DAA can be examined in 3 groups as the right dominant, left dominant, and balanced aortic arch. In line with the information stated in the literature, the right arc is dominant in $75 \%$ of the cases, the left arch is dominant in $20 \%$, and both arch dominance is equal in $5 \%$ of the cases. Our patient had a right dominant aortic arch. If the pressure increases due to the anatomical variation, the patient may develop symptoms of aortic enlargement, rupture, dissection, and compression. Currently, our patient does not have any compression symptoms. To prevent complications that may develop in our patient, we think that it will be useful to monitor the heart functions of our patient with regular ECHO follow-ups and to evaluate them with detailed contrast-enhanced CT if complications are observed. We think we can contribute to the literature by sharing this rare case.

Keywords: double aortic arch, anatomy, variation, congenital vascular malformation

\section{0-53}

\section{A case with Chilaiditi sign, horseshoe kidney and left-sided Bochdalek hernia}

$\underline{\text { Aktaș GD }}{ }^{1}$, Ünver Doğan $N^{1}$, Koplay $M^{2}$, Fazlıogulları $Z^{1}$, Çelik $\mathrm{G}^{3}$

'Departmant of Anatomy, Faculty of Medicine, Selçuk University, Konya, Turkey; ${ }^{2}$ Departmant of Radiology, Faculty of Medicine, Selçuk University, Konya, Turkey; ${ }^{3}$ Department of Internal Medicine, Division of Nephrology, Faculty of Medicine, Selçuk University, Konya, Turkey

Objective: Chilaiditi sign is a rare anomaly defined as the presence of air under the right diaphragm on a radiography. Horseshoe kidney is the most common congenital fusion anomaly of the urinary system and is usually detected inciden- 
tally. With the widespread use of imaging methods, it has been understood that the number of cases with Bochdalek hernia is higher than thought. In this study, we aimed to present a rare case in which horseshoe kidney, Chilaiditi sign and left-sided Bochdalek hernia are seen together.

Case: A 53-year-old male patient who complained of abdominal swelling and coughing had a Chilaiditi sign characterized by an aerial image below the right diaphragm on direct abdominal radiography. Computed tomography of the abdomen showed fusion of the lower poles of the kidneys in the middle line, and a lung graphy showed a hernia of the left posterolateral diaphragm. These images were found to be compatible with horseshoe kidney and Bochdalek hernia, respectively.

Conclusion: Chilaiditi sign is also known as colonic interposition. Recognition of the Chilaiditi sign before colonoscopy and percutaneous transhepatic procedures such as liver biopsy are important for preventing intestinal injury and perforation. Chilaiditi sign, called Chilaiditi syndrome in the presence of an accompanying symptom. The association of this syndrome with a right-sided Bochdalek hernia has been reported in the literature. Horseshoe kidney is usually asymptomatic and half of the patients with horseshoe kidney also have various extrarenal diseases and syndromes. As in this case, it should be borne in mind that pathologies belonging to other systems may develop in patients with one or more abnormalities.

Keywords: chilaiditi sign, horseshoe kidney, Bochdalek hernia, congenital anomaly

\section{0-54}

\section{Pancreas divisum: a case report}

Tunçyürek $\mathrm{Ö}^{1}$, Lafçı Fahrioğlu $\mathrm{S}^{2}$, Yükselen $\mathrm{A}^{3}$

'Department of Radiology, Faculty of Medicine, Near East University, Nicosia, TRNC; ${ }^{2}$ Department of Anatomy, Faculty of Medicine, Near East University, Nicosia, TRNC; ${ }^{3}$ Department of Gastroenterology, Faculty of Medicine, Near East University, Nicosia, TRNC

Objective: The pancreas divisum is the most common pancreatic ductal anomaly. It occurs because of the fusion failure of the ventral and dorsal pancreatic buds in the 2 nd months of the embryological development in $4-10 \%$ of the population. Pancreas divisum is classified as a fusion anomaly of the pancreas. The most of the pancreas divisum is usually asymptomatic but some of them may cause pancreatic and peripancreatic diseases.

Case: A 54-year-old female patient was admitted to our hospital for routine check-up. The Type I (complete type) pancreas divisum was detected by the magnetic resonance imaging performed due to high levels of direct bilirubin. Magnetic resonance images revealed that the short ventral duct of the pancreas was opening into the major duodenal papilla, and the longer dorsal duct was opening into the minor duodenal papilla.

Conclusion: Type I pancreas divisum is the most prevalent PD subtype with incidence of $70 \%$ in whole PD cases. Having information about the embryology and anatomical variations of the pancreas will be very useful and guiding in invasive or non-invasive interventions that should be applied to possible pancreas pathologies for diagnosis and treatment including surgery.

Keywords: pancreas divisum, Type I pancreas divisum, magnetic resonance imaging, high levels of the direct bilirubin

\section{0-55}

Morphometric analysis and surgical importance of the palatum durum

Erdem ${ }^{1}$, Şenol RGT ${ }^{2}$, Boyan $\mathrm{N}^{1}$, Oğuz Ö

'Department of Anatomy, Medical Faculty, Çukurova University Adana, Turkey; ${ }^{2}$ Department of Anatomy, Medical Faculty, Bolu Abant izzet Baysal University, Bolu, Turkey

Objective: The palatum durum, which plays an important role in passive articulation, is important in developmental defects, trauma and palatal surgery. In this study, it was aimed to determine the variational morphometric characteristics of palatum durum.

Methods: Measurements were performed with a digital sliding caliper accurately to $0.1 \mathrm{~mm}$, on 33 craniums belonging to the Çukurova University Medical Faculty, Department of Anatomy. Ages and genders were unknown of the specimens. The palatum durum types were classified according to the palatine index formula (palatum durum breadth/palatum durum lengthX100). The conformation of the data to normal distribution was analyzed by the Kolmogorov-Smirnov test. Bilateral (right-left) comparison of normally distributed data were investigated by dependent samples t-test (paired t-test). The relationship between measurement parameters was evaluated by Pearson's correlation. The significance level was determined as $\mathrm{p}<0.05$. Statistical analysis was performed with SPSS 20.0.

Results: Length and breadth of the palatum durum were $51.69 \pm 3.71 \mathrm{~mm}$ and $30.54 \pm 3.61 \mathrm{~mm}$, respectively; length of the premaxilla and proc. palatinus were $6.29 \pm 1.52 \mathrm{~mm}$ and $6.29 \pm 1.52 \mathrm{~mm}$, respectively; the distance between foramen palatinum majus and sutura palatina mediana on right and left sides were $17.02 \pm 1.69 \mathrm{~mm}, 16.94 \pm 1.81 \mathrm{~mm}$, respectively. While $45.5 \%$ single and $42.4 \%$ double foramina palatina minora were observed on the right side, it was not seen in $6.1 \%$ at all. On the left side, these percentages were determined as $54.5 \%, 24.2 \%$ and $3 \%$, respectively. According to palatine index data, palate types were found as: $66.6 \%$ leptostaphyline (n: 22), 9.9\% mesostaphyline (n: 3), 24.2\% brachystaphyline (n: 9). There was no significant difference between bilateral measurements ( $>0.05$ ). In our measurement parameters, the highest correlation was determined between the palatum durum length and the proc. palatinus length $(r=0.820, p<0.001)$. Also there were significantly high correlated data observed between the various measurements in this study $(\mathrm{r}=0.458-0.700, \mathrm{p}<0.01)$ : between the palatum durum breadth and the right and left foramen palatinum majus-sutura palatina mediana measurements; between the palatum durum length and the proc. palatinus length measure- 
ments; between the measurements of the foramen palatinum majus-sutura palatina mediana in the right and left sides.

Conclusion: We think that our results can help to determine the differences between the races in anthropological studies. We also believe that our results may also be a guide in nerve block anesthesia to be applied at the foramen palatinum majus level in palatal surgeries.

Keywords: foramen palatinum majus, palatal surgery, palatine index, palatum durum

\section{0-56}

Morphometric measurements of palatum durum and palatal index

$\underline{\text { Uzabaci H}}$, Kafa IM

Department of Anatomy, Faculty of Medicine, Bursa Uludağ University, Bursa, Turkey

Objective: The palatum durum is an important anatomical structure in terms of dental and palate surgery. For example, the foramen palatinum majus is located approximately $0.5 \mathrm{~cm}$ anterior from the posterior edge of the palatum durum and from this point the anesthesia of the posterior part of the palate is performed. With anesthetic procedures performed near the foramen incisivum, the posterior parts of the upper anterior six teeth and the anterior parts of the hard palate can be anesthetized. The aim of this study is to determine the morphometric properties of the hard palate and to obtain quantitative data on its important anatomical features, as well as to calculate the palatal index.

Methods: The study was carried out on 21 contemporary skulls. Measuring the distance between "foramen palatinum majus and sutura palatina mediana", "foramen palatinum majus and fossa incisive", "foramen palatinum majus and spina nasalis posterior", angle measurement between "foramen palatinum majus, fossa incisiva and sutura palatina mediana", using Image J program on two-dimensional digital images of skulls. The palatal index was calculated by measuring the number of lesser palatine foramina and the palate length - palate width (Palatal index = (palate width / palate length) X 100). Independent samples t-test and IBM SPSS 20 program were used for data analysis. The results obtained were interpreted as statistically significant $\mathrm{p}<0.05$.

Results: A significant difference was found in distance between the foramen palatinum majus and the suture palatina mediana. ( $\mathrm{p}=0.019$ ) on the left and right sides. No significant difference was found in the measurement of the distance between the foramen palatinum majus and the fossa incisiva, the angle between the foramen palatinum majus, fossa incisiva and the sutura palatina mediana, and the distance between the foramen palatinum majus and the spina nasalis posterior. While the average number of lesser palatine foramina was $1.952 \pm 0.17$ on the left side, it was $1.714 \pm 0.16$ on the right side. Of the 21 skulls examined in terms of palatal index, 9 were grouped as leptostaphyline $(x<80), 4$ as mesostaphyline $(80 x \leq x<85)$, and 8 as brachystaphyline $(85 \leq x)$.
Conclusion: We believe that our Turkish population specific results will be useful in terms of specific interventions for this region, which will be added to the literature.

Keywords: palatum durum, foramen palatinum majus, fossa incisiva, palatal index

\section{0-57}

Determination of the variations and morphometry of the foramen zygomaticofacialis

Kılıç SN, Erdem H, Boyan N, Oğuz Ö

Department of Anatomy, Faculty of Medicine, Çukurova University, Adana, Turkey

Objective: Foramen zygomaticofacialis is (FZ) localized near the infraorbital margin on os zygomaticum, and the nerves and veins with the same name emerge out through it. Having knowledge about the variations and localizations of this foramen is important in maxillofacial and orbital surgery and in preventing complications. The aim of this study was to determine the variations, localizations, and morphometric features of foramen zygomaticofacialis.

Methods: A total of 28 skulls, with unknown age and gender, belongs to the Çukurova University Medical Faculty, Department of Anatomy were used in this study. Fractured or deformed skulls were not included. Measurements were performed with a digital sliding caliper accurately to $0.1 \mathrm{~mm}$. Statistical analysis was performed with SPSS 20.0. The Shapiro Wilks test was used for assessing normality of variables. In addition to descriptive statistical methods (i.e. mean, standard deviation and percentage), the independent sample t-test was used in the evaluation of the quantitative data that fitted the normal distribution between two sides. The significance level was evaluated at $\mathrm{p}<0.05$. The correlation between variables was analyzed with a Spearman correlation test.

Results: In our study, FZ was detected as follows for right-left side; Type 1: 71.4-50.0\%, Type 2: 14.3-28.6\%, Type 3: $10.7-$ 7.1\%, Type 4: 0-0\%, Type 5: 3.6-14.3\%, respectively. On the right and left sides; the distances between FZ-sutura zygomaticomaxillaris were: $12.34 \pm 2.95 \mathrm{~mm}$; and $11.92 \pm 3.65 \mathrm{~mm}$; FZ-orbita: $6.65 \pm 1.49 \mathrm{~mm}$ and $6.63 \pm 1.52 \mathrm{~mm}$; FZ-sutura frontozygomatica: $26.62 \pm 2.89 \mathrm{~mm}$ and $26.86 \pm 3.73 \mathrm{~mm}$; FZ-zygomatic angle $\mathrm{mm}$ : $13.32 \pm 2.98 \mathrm{~mm}$ and $13.54 \pm 3.64 \mathrm{~mm}$; FZ-sutura temporozygomatica $20.90 \pm 4.98 \mathrm{~mm}$ and $21.35 \pm 4.15 \mathrm{~mm}$; and the lower edge of FZ-os zygomaticum $19.06 \pm 2.79 \mathrm{~mm}$ and $18.69 \pm 2.59 \mathrm{~mm}$, respectively. No statistically significant differences were detected between right and left sides ( $>00.05)$. The strong positive correlation was detected between zygomatic angle and the right and left FZ-sutura frontozygomatica measurements $(\mathrm{p}<0.001)$.

Conclusion: We believe that our results can guide surgical procedures, such as orbitozygomatic craniotomy, involving the precise region and can help to prevent iatrogenic complications. 
Keywords: foramen zygomaticofacialis, naxillofacial surgery, os zygomaticum, ramus zygomaticofacialis nervus zygomatici

\section{0-58}

Morphometric measurements of apertura piriformis, palatum durum and choana on Late Byzantine skulls

Ertan E, Şendemir E

Department of Anatomy, Faculty of Medicine, Bursa Uludağ University, Bursa, Turkey

Objective: It is known that apertura piriformis, choana and airway length are important for physiological nasal breathing. Furthermore, palate index values which are obtained by hard palate morphometry are important in palate surgery and maxilla prostheses. In this study, we aimed to reveal the apertura piriformis and choana diameters and the morphometric properties of the palatum durum in skulls of Byzantine period.

Methods: The study was performed on 22 skulls from the Late Byzantine period which were obtained from the archeological search area of İznik (Nicaea) and kept in our laboratories. Height and width measurements of apertura piriformis, right and left choana height and width measurements, palatum durum length and width were measured using calipers. In the analysis, t-test and Mann-Whitney $\mathrm{U}$ test were used to compare right and left measurement values in independent samples. Pearson correlation analysis was applied to examine the relationship between variables and all analyzes were performed with IBM SPSS Statistics 20 program. The obtained results were interpreted as meaningful $\mathrm{p}<0.05$.

Results: When the values of the right and left apertura piriformis in the skulls of the Late Byzantine era were examined, the mean measurements of the right and left apertura were $34.818 \pm 4.465 \mathrm{~mm}$ for height and $23.681 \pm 1.763 \mathrm{~mm}$ for width. The average values of the length and width of the palatum durum were $50.75 \pm 4.697 \mathrm{~mm}$ and $60.34 \pm 5.37 \mathrm{~mm}$, respectively. Measurement values for the right and left choana in the Late Byzantine era skulls were: right side height $24.676 \pm 2.344 \mathrm{~mm}$, left side height $23.588 \pm 1.822 \mathrm{~mm}$, right side width $13.382 \pm$ $1.352 \mathrm{~mm}$, and left side width $13.558 \pm 1.647 \mathrm{~mm}$.

Conclusion: In this study, morphometric data of apertura piriformis, choana and palatum durum were obtained by examining skulls belonging to the Late Byzantine period that lived in the same geographical region. In the clinic, especially after traffic and sports accidents, the nasal passage bone structures are fractured and surgical treatment is required quickly and it is known that when performing osteotomy in corrective rhinoplasty operations, highly sensitive anatomical knowledge is required regarding apertura piriformis. The obtained morphological data are partake of resources for many disciplines.

Keywords: apertura piriformis, choana, palatum durum, skulls of Late Byzantine period

\section{0-59}

Localization and morphometry of foramen mandibulae in today's and Byzantine period dry mandibles

Güler M, Yildız Yilmaz M, Coşkun I

Department of Anatomy, Faculty of Medicine, Bursa Uludağ University, Bursa, Turkey

Objective: In this study, it was aimed to compare the data related two different periods by measuring the foramen mandibulae, which is important for mandibular anesthesia and surgical interventions in today's and Byzantine period dry mandibles.

Methods: The study was carried out on a total of 40 dry mandibles, 20 from today and 20 from the Byzantine Period. ImageJ program was used on two dimensional digital images taken from the mandible. In the mandibles belonging to two periods, the distances of the right and left foramen mandibulae to the angulus mandibulae, incisura mandibulae, processus coronoideus, ramus mandibulae anterior, ramus mandibulae posterior, caput mandibulae and spina mentalis were measured. T test and Mann-Whitney $\mathrm{U}$ test were used for data analysis and independent samples, and IBM SPSS Statistics 20 software was used for analysis.

Results: In today's bones, the distance between the foramen mandibulae and the incisura mandibulae on the left $(\mathrm{p}=0.049)$; the distance between foramen mandibulae and processus coronoideus both on the right $(\mathrm{p}=0.041)$ and on the left $(\mathrm{p}=0.007)$; the distance between the foramen mandibulae and the caput mandibulae on the left side $(\mathrm{p}=0.004)$ were found to be longer than Byzantine bones. The distance between the foramen mandibulae and ramus mandibulae anterior was found to be longer on the right side $(\mathrm{p}=0.017)$ than on the left side in Byzantine bones.

Conclusion: In this study, the morphometric data of the foramen mandibulae were collected by examining the dry mandibles of two different periods that lived in the same geographical region. It may be beneficial to consider the longer distances in today's mandibles in maxillofacial surgery applications which we think might have been caused by eating habits. Keywords: foramen mandibulae, mandible, osteology, Byzantine period

\section{0-60}

An essay on gender prediction using machine learning algorithms and parameters obtained from computed tomography images of the cranium skeleton

Toy $S^{1}$, Seçgin $Y^{1}$, Öner $Z^{1}$, Turan $K^{2}$, Öner $S^{3}$

'Department of Anatomy, Faculty of Medicine, Karabük University Karabük, Turkey; '2Department of Medical Biology and Genetics, Faculty of Medicine, Karabük University, Karabük, Turkey; ${ }^{3}$ Department of Radiology, Faculty of Medicine, Karabük University, Karabük, Turkey

Objective: Gender prediction is one of the most critical issues in forensic medicine and anthropology. Gender prediction 
provides rapid decision-making by reducing the probable identity probabilities by almost half as it constitutes the lowest level of other identity findings (age, lineage, height, weight). The aim of this study is to predict gender using machine learning algorithms with parameters belonging to the cranium skeleton obtained from Computed Tomography (CT) images.

Methods: CT images of 150 women and 150 men (300 in total) between the ages of 20 and 65 years, consisting of individuals without any pathology in the cranium skeleton or a previous surgical operation, were collected from the Picture Archiving and Communication System of the hospital. Later, images were transferred to the Horos Medical Image Viewer (Version 3.0, USA) program, which is a personal workstation in Digital Imaging and Communications in Medicine (DICOM) format, and length, angle, curvature length, and area units were obtained in accordance with the determined anatomical points. The accuracy of estimating gender has been demonstrated by using the obtained measurements in the input of machine learning algorithms.

Results: The data in our study were analyzed using machine learning algorithms such as Logistic Regression (LR), Linear Discriminant Analysis (LDA), Quadratic Discriminant Analysis (QDA), Random Forest (RF), Extra Trees Classifier (ETC), Decision Tree (DT). At the end of the analysis, an accuracy ratio was 0.90 with $\mathrm{LR}, 0.88$ with $\mathrm{LDA}, 0.83$ with QDA, 0.88 with RF, 0.85 with ETC, and 0.81 with DT.

Conclusion: In our study, a sensitive, modern imaging method such as CT, which is not affected by orientation and allows to make images orthogonal, was preferred instead of measurements made with direct osteometric devices (calipers, odontometers, digital distance meters) which are traditionally used for gender prediction. In addition, in our study, a more up-todate approach has been put forward by using machine learning algorithms, which have recently been used in medicine.

Keywords: cranium, gender prediction, computed tomography, machine learning algorithms

\section{0-61}

Three dimensional distal radius bone modeling by using computed tomography images

İsmailoğlu $\mathrm{P}^{1}$, Kahraman Yildırım $\mathrm{FG}^{2}$

'Department of Anatomy, Faculty of Medicine, Acıbadem Mehmet Ali Aydınlar University, Istanbul, Turkey; '2Department of Anatomy, Cerrahpaşa Medical Faculty, Istanbul University-Cerrahpaşa, Istanbul, Turkey

Objective: Racial differences in distal radius morphology are important in hand surgery. This study aims to investigate the morphology of the distal radius and contribute to the reconstruction of the anatomical alignment in distal radial fractures.

Methods: Bone modeling of 6 radius CT images with preserved anatomical integrity was performed with Invesalius,
Blender, Meshmixer programs and a 3D printer named Zortrax M200. The angle of the carpal articular surface was measured in the Sectra as Scaphoid Facet Inclination (SFI), Lunate Facet Inclination (LFI). For volar curvature a sagittal section was taken from the midpoint of the scaphoid facet parallel to the radius body. The angle between the volar curvature and radial body on the sagittal section was defined as A1. The same procedure was made for the midpoint of the lunate facet and the angle between the radius body on the sagittal section was named A2. A3 was recorded as the angle measured in the sagittal section taken from the line between the scaphoid and lunate facet. The widest part of the volar surface was expressed as the width $(\mathrm{G})$ of the transverse line drawn from the lower edge of the ulnar notch to the outer side. The SPSS 26 program was used for descriptive and comparative analysis for the measured parameters A1, A2, A3, G. Of the 6 radii CTs modelled, 4 were right side and 2 were left side. And 5 were male and 1 female CT. At the same time, 3 of them were from the Anatolian society and the rest belonged to foreigners whose origins were unknown. Racial differences as domestic and foreign CT were evaluated using the Mann-Whitney $U$ test, one of the nonparametric tests.

Results: The mean G value was $27.6 \mathrm{~mm}$; average SFI 25.8 degrees; average LFI -0.96 degrees. Average A1 was found to be 35 , average $\mathrm{A} 2$ was 25.7 , average $\mathrm{A} 3$ was 33.6 degrees. There was a significant difference for A1 between the local and foreign groups $(\mathrm{p}<0.05)$. There was no significant difference in $\mathrm{A} 2, \mathrm{~A} 3$ and $\mathrm{G}$ values in terms of race and age variables ( $>0.05)$. However, a significant positive correlation was found between age and $\mathrm{G}$ value $(\mathrm{p}=0.042)$.

Conclusion: The distal radius modeling measurements show that there are morphological differences for the race. Due to these differences, it is thought that an individual-specific reconstruction is required to restore anatomical alignment in wrist fracture surgery.

( ${ }^{*}$ Project Support ABAPKO Decision No: 2020/03/04)

Keywords: radius, morphology, 3D modeling, orthopaedic surgery

\section{0-62}

Deep learning and its application in medical studies

Öztürk K

Department of Anatomy, Faculty of Medicine, Süleyman Demirel University, Isparta, Turkey

It is an artificial intelligence method used in the solution of deep learning classification and regression problems and obtained by deepening the architecture used in artificial neural networks. It is used in many areas such as face recognition and license plate recognition. Similar to how neurons communicate with each other, in deep learning architecture, neuron-like structures are interrelated. It is thought that the algorithm can have the capac- 
ity of learning, memorizing and relating in this way. Unlike traditional machine learning methods, deep learning takes images directly as input, learns the distinctive features required for classification by itself and can give high accuracy rates in predicting what they have learned. With the active introduction of artificial intelligence into our lives, the use of systems that can support physicians by performing disease diagnosis is becoming widespread. These systems have been found to perform similar or better to specialist physicians. Thus, it is aimed to eliminate the disadvantages caused by the fatigue factor in the diagnosis of diseases. In addition, it is aimed to overcome problems such as making different diagnoses for the same diseases that may arise from the inexperience of physicians. When we look at the studies of diagnosis of diseases with deep learning, we come across many different studies. In a study on the early diagnosis of Alzheimer's disease, it was observed that the radiological images of the control group and Alzheimer's patients were successfully differentiated. A group of researchers developed a system to detect bone fractures and cracks, which are often needed in hospital emergency rooms, using deep learning. They aimed to minimize situations such as cracks, which can be overlooked and cannot be diagnosed early, due to the absence of a specialist physician in emergency departments all the time and sometimes workload. In addition, the deep learning method has been used with a high success rate in many studies such as detection of cataracts, tumors, internal bleeding and malaria, classification of ECG arrhythmias and skin cancers, determination of bone age and sex estimation from hand radiographs. Considering the high success rates of deep learning in medical studies so far, it is seen that its use in this field will continue to become widespread. It is seen as a method that can be used in the detection of anatomical variations as well as in anatomical studies such as distinguishing organs in radiological images, age and sex determination.

Keywords: algorithm, artificial intelligence, artificial neural networks, machine learning

\section{0-63}

\section{Augmented reality in anatomy education in the Covid-19 period}

Çelik Z, Ilgaz HB

Department of Anatomy, Faculty of Medicine, Hacettepe University, Ankara, Turkey

Objective: Most countries around the world have temporarily closed educational institutions to control the spread of Covid-19 outbreak. With faculties closed due to Covid-19, education has now become online in many places, but it is still debatable whether only online education will be sufficient for laboratoryrequired courses such as anatomy. Virtual training appears to be the only form of training in the current scenario. Modern methods for learning anatomy are currently in the form of augmented reality (AR) and virtual reality (VR). AR allows you to obtain a $3 \mathrm{D}$ image of cadavers and mock-ups in the anatomy laboratory by transferring them to a virtual environment. VR systems can be used to provide effective and efficient medical training as an alternative to cadavers for training. Despite the challenges in education, the pandemic creates opportunities for faster adoption and development of technologies.

Methods: A literature review was performed. Research has been conducted in PubMed, Wiley Online Library, American Association for Anatomy, and Science Direct databases.

Results: Generation Y students preferred web-based, selflearning and media-following skills, simulations, interactive group activities, and game-style information presentations. A study using AR to teach head-neck anatomy to first-year medical students showed that the success rate increased from $59 \%$ to $95 \%$. In a study of 72 students using cadavers and 3D learning the upper limb bones, students using 3D software performed better. Another study shows that VR is more effective in learning human heart anatomy, especially for people with low basic knowledge. The "Virtual Human Dissector" software was compared with traditional learning methods, and the results showed that using the software was effective as studying cadavers in the dissection room.

Conclusion: In the reviewed studies, it has been shown that students look at a model from various angles and contribute to their anatomical learning through their visual spatial skills. It has been found that the use of 3D digital models does not require guidance, and students can easily use them. 3D applications are more preferable in terms of time-limitation, cadaver difficulties and lack of student timidity. The use of visual aids is a highly effective teaching method, and researches show increasing memory retention compared to traditional teaching methods. AR has proven to be a fairly acceptable and fun anatomy teaching tool. While the value of cadaver dissection is irreplaceable, digital technology can greatly facilitate learning anatomy.

Keywords: covid-19, practical anatomy training, augmented reality, virtual reality

\section{0-64}

\section{Evaluation of septum pellucidum morphometry}

Alkan $\mathrm{E}^{1}, \ddot{O ̈ z ~ N}^{1}$, Karaali $\mathrm{K}^{2}$, Erdoğan A3 , Süzen $\mathrm{LB}^{4}$

'Department of Anatomy, Faculty of Medicine, Akdeniz University, Antalya, Turkey; 'Department of Radiology, Faculty of Medicine, Akdeniz University, Antalya, Turkey; ${ }^{3}$ Department of Psychiatry, Faculty of Medicine, Akdeniz University, Antalya, Turkey; ${ }^{4}$ Department of Basic Sciences, Faculty of Dentistry, Antalya Bilim University, Antalya, Turkey

Objective: Brain midline structures are important information providing points in evaluating brain development. Septum pellucidum(SP) is located in the midline between the cornu anterior and pars centralis of both ventriculus lateralis. It is attached to inferior and posterior faces of the corpus callosum at above and front, and anterior surface of fornix at below and back, forming a sail-like shape, wide anteriorly and narrow pos- 
teriorly. It is a transparent thin structure formed by fusion of two lamina septalis. It contains nerve fibers, substantia grisea and neuroglia. The walls facing the ventricles are paved with ependymal cells. In studies, pathologies such as agenesis, cyst of SP have been associated with neurological and psychiatric diseases. Its location, adjacent structures and structure are important in terms of surgery. In this study, we aimed to define morphological properties of septum pellucidum.

Methods: Healthy individuals who applied to the outpatient clinic and had no pathology on magnetic resonance (MR) images were included in our study. Ten males ages between 21-49 (mean:33) and ten females ages between 21-44 (mean: 30) were studied. Measurements were made in T1-weighted midsagittal and transverse sections with instruments included in the imaging program (SECTRA). In SWI sequence images, Total Intracranial Volume (TIV) was calculated automatically with CAT tools integrated into the SPM12 application package in the MATLAB (R2020b) program. The antero-posterior length between the back of genu corporis callosi and the intersection of corpus fornicis with truncus corporis callosi, height at foramen interventriculare level and area of septum pellucidum were measured. Thickness in transverse section at foramen interventriculare level and in transverse section passing through the base of the truncus corporis callosi, was measured and averaged.

Results: The averages and standard deviations of manually measured parameters of SP are $37.91 \pm 6.70 \mathrm{~mm}$ for female, $35.46 \pm 4.77$ $\mathrm{mm}$ for male individuals for length; $7.10 \pm 3.41 \mathrm{~mm}$ for female, $9.80 \pm 2.47 \mathrm{~mm}$ for male individuals for height; $2.66 \pm 0.18 \mathrm{~mm}$ for female, $2.53 \pm 0.36 \mathrm{~mm}$ for male individuals for thickness; $284.65 \pm 106.23 \mathrm{~mm} 2$ for females and $265.07 \pm 89.83 \mathrm{~mm} 2$ for males for the area. When the manually measured values were compared according to gender, no statistically significant difference was found. When the ratio of SP's area to TIV was compared according to gender, no statistically significant difference was found.

Conclusion: We think that SP morphology is important in planning surgery to be performed in pathologies such as ventriculoperitoneal shunt and ventricular cyst. In addition, we think that knowing normal morphological boundaries will contribute to etiology of diseases such as psychosis, depression which may have a neuropsychological basis.

Keywords: septum pellucidum, morphometry, mid-line brain anomalies, neurosurgery

\section{$0-65$}

Evaluation of the location of the sutura temporozygomatica on the arcus zygomatica by three dimensional reconstruction method

Atalar $\mathrm{K}^{1}$, Özer $\mathrm{CM}^{1}$, Y1lmazer Kayatekin AZ ${ }^{1}$, Keskin $\mathrm{N}^{2}$

'Department of Anatomy, Faculty of Medicine, Zonguldak Bülent Ecevit University, Zonguldak, Turkey; 'Department of Anatomy, Institute of Health Sciences, Kocaeli University, Kocaeli, Turkey
Objective: Os zygomaticum is located in an important position connecting the facial skeleton to the cranium. It also has important functions, as some of chewing and mimic muscles originate from there. Arcus zygomaticus formed by the union of processus temporalis of os zygomaticum and processus zygomaticus of os temporale through the sutura temporozygomatica. This structure is also aesthetically important in facial appearance. In this study, it was aimed to investigate whether the anatomical structure of arcus zygomaticus and sutura temporozygomatica differ according to gender, age and side parameters.

Methods: In our study, the images of individuals older than 18 years of age, who were taken cranial computed tomography (CT) for any reason in the Department of Radiology, Faculty of Medicine, Zonguldak Bülent Ecevit University since 01.01.2018, were evaluated retrospectively. CT images were transferred to Osirix MD software and three-dimensional reconstruction is performed. The level at which arcus zygomaticus can be observed on the three-dimensional images obtained was adjusted and measurements were made.

Results: This study performed on 50 individuals, 27 males and 23 females, with a mean age of 42 . The distance between the most lateral point of the right arcus zygomaticus and the linea mediana measured $6.55 \mathrm{~cm}$ in average. The distance between the most lateral point of the left arcus zygomaticus and the linea mediana measured $6.75 \mathrm{~cm}$ on average. The projective length of arcus zygomaticus measured as $4.64 \mathrm{~cm}$ on the right side and $4.60 \mathrm{~cm}$ on the left side on average. The average length of processus zygomaticus measured $3.69 \mathrm{~cm}$ on the right side and $3.51 \mathrm{~cm}$ on the left side. The average length of processus temporalis measured $0.94 \mathrm{~cm}$ on the right side and $1.09 \mathrm{~cm}$ on the left side.

Conclusion: Skull bones, especially in the field of forensic medicine, are often used in gender determination by evaluating them with morphological and morphometric analysis methods. Today, anthropological evaluations can be made on imaging methods such as CT and MRI. In this way, it is possible to make examinations in a much shorter time without the need for maceration. Anthropometric and morphological evaluations of sutura temporozygomatica and arcus zygomaticus can be used in interventional and surgical procedures, anthropological studies and forensic studies to be performed on the face.

Keywords: sutura temporozygomatica, arcus zygomaticus, three-dimensional reconstruction

\section{0-66 \\ Investigation of fossa navicularis on cone-beam computed tomography images and dry bones}

$\underline{\text { Adanır SS }}{ }^{1}$, Kervancıoğlu $\mathrm{P}^{1}$, Orhan $\mathrm{M}^{1}$, Bahşi $\dot{\mathrm{I}}^{1}$, Yalçın ED ${ }^{2}$

'Department of Anatomy, Faculty of Medicine, Gaziantep University, Gaziantep, Turkey; ${ }^{2}$ Department of Oral Diagnosis and Radiology, Faculty of Dentistry, Gaziantep University, Gaziantep, Turkey 
Objectives: Fossa navicularis $(\mathrm{FN})$ is a bone depression, is not always present, located in the external surface of cranial base, in front of foramen magnum and pharyngeal tubercle. In literature, $\mathrm{FN}$ is known by different names such as fossa navicularis magna, medial basal fossa, and pharyngeal fossa. FN can create an infection spread path from pharynx to intracranial structures. In this study, it was aimed to investigate the incidence of $\mathrm{FN}$ on cone-beam computed tomography (CBCT) images of the healthy Turkish population and dry bones and to examine the morphometric and morphological properties of the detected FN.

Methods: CBCT images of 755 individuals aged 18 and over who applied to Gaziantep University Faculty of Dentistry for any reason, who did not have any trauma and pathology history of cranial base and whose pathology was not detected in this region, and also 33 skulls and 11 bone specimens contain occipital and sphenoid bones which unknown age, gender and ethnic characteristic which present in Anatomy Department of Gaziantep University Faculty of Medicine were examined. The incidence of $\mathrm{FN}$ was determined by evaluating the CBCT images using Planmeca Promax 3D (with Romexis software version 3.2.0). Sagittal and transverse diameters, depth, and distance of FN to cranial cavity were measured in images. These parameters were compared between genders. In dry bones, sagittal and transverse diameter and depth of FN were measured using calipers. In addition, FN were classified as shape both in the CBCT images and dry bones.

Results: The CBCT images of 755 individuals in total, four hundred and fifteen males (mean age:43.20 15.12 ) and 340 (mean age:42.64 \pm 14.73 ) females, were examined. $\mathrm{FN}$ was detected in 88 of 755 (11.65\%) CBCT images and 10 of 44 $(22.72 \%)$ dry bones. Average values of sagittal, transverse diameters, depth, and distance to cranial cavity of $\mathrm{FN}$ detected in CBCT images were as $5.68 \pm 2.22,5.63 \pm 1.65,2.00 \pm 0.48$ $6.60 \pm 2.17 \mathrm{~mm}$, respectively. It was determined that 69 (78.41\%) of the FN were oval and 19 (21.59\%) of them were round. Depth of $\mathrm{FN}$ was found to be statistically higher in females than males $(\mathrm{p}=0.043)$, and other parameters did not show a statistically significant difference between genders. Average values of the sagittal, transverse diameters and depth of $\mathrm{FN}$ detected in dry bones were found to be as $1.44 \pm 0.30$, $1.42 \pm 0.35$ and $1.63 \pm 0.50 \mathrm{~mm}$, respectively. It was determined that 7 of $10 \mathrm{FN}$ detected in skulls were oval (70\%) and 3 of them were round $(30 \%)$.

Conclusion: It is seen that there are quite a few studies in the literature examining the morphometric properties of $\mathrm{FN}$. Because of its proximity to important anatomical structures and its clinical importance, it is thought that well-known anatomic variations such as $\mathrm{FN}$ will be significant in pathologies of this region.

Keywords: anatomic variation, cone beam computed tomography, fossa navicularis, morphometry

\section{0-67}

Examination of thorax morphology by multi-slice computed tomography in individuals with pectus excavatum

Atçeken $A^{1}$, Fazlıŏulları $Z^{1}$, Can A ${ }^{2}$, Ünver Doğan $\mathrm{N}^{1}$, Karabulut AK

'Department of Anatomy, Faculty of Medicine, Selçuk University, Konya, Turkey; 'Department of Thoracic Surgery, Faculty of Medicine, Selçuk University, Konya, Turkey

Objective: In our study, it was aimed to compare the relationship between morphometric measurements of the thorax with pectus excavatum types in individuals with pectus excavatum, to compare the differences of these types by gender, and to compare the values of the obtained data before and after puberty.

Methods: In the study, data of 204 patients with pectus excavatum who applied to the Selçuk University Medical Faculty Department of Thoracic Surgery were retrospectively analyzed. Thoracic computed tomography images of 100 patients, 84 males, and 16 females, aged between 4 and 56 were included in the study. Pectus excavatum types were evaluated according to 7 types mentioned in the literature and different types were added to these types. The pectus indices, asymmetry indices, flatness indices, vertebral indices, frontosagittal indices, sternal torsion angles, and angle of Louis of patients were calculated.

Result: The pectus excavatum types of the cases respectively; group 1A was seen in 27 patients, group 1B was seen in 53 patients, group 2B was seen in 9 patients, group $2 \mathrm{C}$ was seen in 8 patients, group 2D was seen in 1 patient and group $2 \mathrm{E}$ was seen in 2 patients. In group 1C, 2 patients with group $1 \mathrm{~B}$ and 1 patient were seen with group $2 \mathrm{~B}$. In group $2 \mathrm{~A}, 1$ patient was seen with group $2 \mathrm{E}$. In male patients, group $1 \mathrm{~A}$, group $1 \mathrm{~B}$, group $2 \mathrm{~B}$, group $2 \mathrm{C}$, group $2 \mathrm{D}$, and group $2 \mathrm{E}$ were found in $20,46,7,8,1$, and 2 individuals, respectively. When the pectus index, asymmetry index, and sternal torsion angle were compared between the prepubertal and postpubertal groups, no significant difference was observed. When the group with a positive sternal torsion angle and a negative sternal torsion angle were compared, it was found that the asymmetry index was statistically significantly higher in the positive oriented group.

Conclusion: The most common group in pectus excavatum types is group $1 \mathrm{~B}$, which is typically asymmetrical. The number of male patients is higher than women in all groups. In addition to previous studies, in our study, an additional type with clockwise sternal torsion characterized by a bulge at the left chest wall was observed. Since the pectus index and vertebral index are important in the surgical indication of pectus excavatum, we think that the study will guide thoracic surgeons.

Keywords: pectus excavatum, anatomy, thorax, morphometry 


\section{0-68}

Relationship between thoracic inlet size and clavicle morphology

Şahin Ş

Department of Radiology, Adlyaman Training and Research Hospital, Adıyaman, Turkey

Objective: Many vital anatomical structures pass through thoracic Inlet (TI). Thoracic inlet size may be important in surgery and complications of these organs. Our aim is to reveal whether there is a relationship between clavicle morphology and TI size.

Methods: Computed tomography images of the right clavicles and TI area of 50 female and 50 male cases were evaluated retrospectively. The length, medial, mid and lateral diameters of the clavicle and the TI area were measured. The clavicle morphology and TI area relationship was evaluated with Pearson's correlation coefficient. In addition, differences in gender among the measured values were evaluated.

Results: There is a strong positive correlation between the clavicle length and TI area $(\mathrm{r}=0.905, \mathrm{p}<0.001)$. The medial and lateral diameter of the clavicle and the TI area have a moderate positive correlation $(\mathrm{r}=0.616, \mathrm{p}<0.001$ and $\mathrm{r}=0.689$, $\mathrm{p}<0.001$, respectively). On the other hand, there is a weak correlation with the diameter of the clavicle middle part $(\mathrm{r}=0.375$, $\mathrm{p}<0.001)$. The clavicle is shorter in women than men, and thinner in the medial, middle and lateral parts. In addition, the area of TI is smaller in women.

Conclusion: Among the clavicle parameters that shows the strongest correlation with TI area is clavicle length.

Keywords: Thoracic inlet; clavicle; correlation; computed tomography

\section{0-69}

The dimple which is the symbol of beauty as aesthetic is variation or anomaly?

Kastamoni Y

Department of Anatomy, Faculty of Medicine, Süleyman Demirel University, Isparta, Turkey

Dimples, seen as a fascinating feature of facial beauty, are an important factor for expressing thoughts and emotions. The most common are cheek and chin dimples. Other dimples seen in the human body; zygomatic dimple, shoulder dimple, elbow dimple, back dimple (venus dimple), gluteal dimples and sacral dimples. Dimples are a genetic trait that follows an autosomal dominant inheritance pattern. If there is a dimple in one of the parents, the probability of the person inheriting the gene is $25-50 \%$, while if both parents have dimples, this rate is between $50-100 \%$. Some dimples are permanent, while others are temporary. The increase in subcutaneous adipose tissue causes disappearing of dimple, while the shortening and elongation in structures such as muscle, skin and ligament can cause the changing of dimple. This explains why some dimples are more common and more noticeable in younger age groups. Cheek dimple, also known as laughing dimple, contributes to facial beauty and expression. It is more common in infants and children of 7-15 years of age compared to adults. It can be unilateral as well as double sided. Anatomically, it is thought that the cheek dimple originates from the double or bifid zygomaticus major muscle, and the muscle fibers belonging to the termination site enter the dermis and cause the dimple. The chin dimples are not related to the facial muscles. It is caused by incomplete fusion of the right and left sides of the mandible or muscles in the region during embryological development. There are also dimples that develop pathologically. Zygomatic dimple is a pathological condition that develops in the tissues squeezed between the zygomatic bone and the uterine wall during fetal development. Similarly, the shoulder dimple is thought to be caused by tissues compressed between the shoulder bones and the uterine wall. Sacral dimple, which is another pathological dimple, is also known as "sacrococcygeal or coccygeal dimple". It is the most common cutaneous anomaly detected in neonatal spinal examination. These dimples larger than $5 \mathrm{~mm}$ and located further from the anus indicate the presence of hidden neural tube defects. If the dimple occurs as a result of an error in the developmental stages and does not represent a pathological condition after birth, it is a variation; however, if it occurs as a result of a trauma during development or if it refers to a pathology after birth, we can say that it is an anomaly.

Keywords: dimple, zygomaticus, bifid, variation

\section{0-70}

Sex determination trial with the proportion of distal phalanx to metacarpals length using direct hand x-ray images

Özsoy $\mathrm{T}^{1}$, Öner $\mathrm{Z}^{2}$, Büyükertan $\mathrm{M}^{3}$

${ }^{\prime}$ Autopsy Assistance Program, Hamidiye Health Services Vocational School, University of Health Sciences, Istanbul, Turkey; ${ }^{2}$ Department of Anatomy, Faculty of Medicine, Karabuk University, Karabuk, Turkey, ${ }^{3}$ Department of Anatomy, Hamidiye Faculty of Medical, University of Health Sciences, Istanbul, Turkey

Introduction: Gender analysis is one of the most important and comprehensive subjects of anthropology and forensic science. Among the anthropometric methods used in gender analysis, osteometry is a widely preferred method because of its easy and cheap applicability. Most of the skeletal structures that make up the body parts, especially the pelvis and all the cranium bones, are the most preferred bones in sex determination.

Objective: In this research, the ratio between the length of distal phalanx and metacarpals calculated and its usability in sex determination are examined through direct hand X-ray images. 
Methods: In this study, X-ray images of 60 individuals $(30 \mathrm{~F}$, $30 \mathrm{M}$ ) between the ages of 20-40 were imaged with the help of Horos program and the distal phalanx and metacarpals were measured and their ratio to each other was calculated. Discriminant Analysis Test was applied to the data and its usability in sex determination was evaluated.

Results: According to the measurement results (from first finger to fifth finger), the mean distal phalanx lengths were measured as $2.53 \mathrm{~cm}, 1.84 \mathrm{~cm}, 1.95 \mathrm{~cm}, 2.02 \mathrm{~cm}, 1.80 \mathrm{~cm}$ in men and 2.25 $\mathrm{cm}, 1.62 \mathrm{~cm}, 1.73 \mathrm{~cm}, 1.78 \mathrm{~cm}, 1.59 \mathrm{~cm}$ in women, respectively. The average lengths of metacarpal bones were measured as 5.02 $\mathrm{cm}, 7.33 \mathrm{~cm}, 7.09 \mathrm{~cm}, 6.30 \mathrm{~cm}, 5.77 \mathrm{~cm}$ in men and $4.51 \mathrm{~cm}$, $6.77 \mathrm{~cm}, 6.52 \mathrm{~cm}, 5.78 \mathrm{~cm}, 5.25 \mathrm{~cm}$ in women, respectively. It was found that phalanx distalis and ossa metacarpi lengths are longer in males than females. When the proportion of phalanx distalis to ossa metacarpi was examined with Discriminant Analysis, it was seen that it correctly predicted males and females at a rate of $63.3 \%$.

Conclusion: As a result of the investigations made using Discriminant Analysis, it is observed that it is possible to determine the gender with the ratio of distal phalanx length to metacarpals length, but the discrimination power is not as high as the pelvis and cranium bones. We think that the discrimination power can be increased if the sample size is increased and the Artificial Neural Network method is used.

Keywords: phalanges; metacarpals; length; gender analysis; discriminant analysis

\section{0-71}

Foot measurements and analyzing foot structure with plantogram in university students

\section{Doğancı ÖI}

Department of Anatomy, Residency Thesis, Istanbul, Cerrahpaşa Medical Faculty, Istanbul University-Cerrahpaşa, Istanbul, Turkey

Objective: This study aims to evaluate foot measurements and foot structure with a plantogram of 18-21 year old university students to scientifically define measurements for shoe lasts of these individuals and to determine pes planus frequency in this age range. Body measurements are affected by socioeconomic conditions like gender, genetics, geographical conditions, nutrition, and living conditions. Defining differences between standards for each population has importance in fields like internationally traded medical products, textile, furniture, machine industry etc.

Methods: Direct measuring with caliper, measuring tape and precision bascule is used. Harris Mat is used to take footprints for plantogram. 300 people (159 women and 141 men) are included. Height, weight, foot length, foot width, foot height, talocalcaneal distance, bimalleolar distance, metatarsophalangeal circumference, tarsal circumference, talocalceneal circumference, and bimalleolar circumference are measured.
Results of the plantogram are interpreted using the Staheli Index and Chippaux-Smirak Index to define pes planus. Results are evaluated with proper statistical methods. All statistical analyzes were analyzed and reported in IBM SPSS Statistics 22.0 program at $95 \%$ confidence level and $\cdot=0.05$ significance level. The distribution of the data was examined with Shapiro Wilk test. Mann-Whitney U test was used to compare two groups of data that did not show normal distribution. Spearman Correlation coefficient was used to compare the relations between foot measurements

Results: Men have significantly larger foot and higher pes planus ratio than women. Two diagnostic indexes for pes planus have been compared.

Conclusion: These indexes aren't reliable alone and when diagnosing pes planus one should back these results with physical examination and clinical findings

Keywords: foot, anthropometry, pes planus

\section{0-72}

\section{Comparison of classical methods for bone age determination with capitohamate planimetry on wrist x-ray}

$\underline{\text { Yildız } \mathrm{H}^{1}}$, Ünver Doğan $\mathrm{N}^{1}$, Öztürk $M^{2}$, Fazlıoğulları $Z^{1}$, Korez $\mathrm{KM}^{3}$, Karabulut $\mathrm{AK}^{1}$

'Department of Anatomy, Faculty of Medicine, Selçuk University, Konya, Turkey; '2Department of Radiology, Faculty of Medicine, Selçuk University, Konya, Turkey; ${ }^{3}$ Department of Biostatistic, Faculty of Medicine, Selçuk University, Konya, Turkey

Objective: The aim of the study is to compare the GreulichPyle and Tanner-Whitehouse methods, which are used in the left wrist radiography for bone age determination in the pediatric age group, with the Capitohamate planimeter method, due to the importance of determining the bone age in the pediatric period.

Methods: In the study, direct radiographs of 210 female and 291 male individuals between the ages of $0-18$ and without any pathology in the left wrist bones obtained in the Radiology Department of Selçuk University Faculty of Medicine Hospital were examined retrospectively. Patients older than 18 years old, those with wrist trauma, those with metabolic diseases and those with congenital anomalies were not included in the study. In the study, 500 os capitatum and os hamatum are as on AP direct graphy images were measured by Greulich-Pyle, Tanner Whitehouse and Capitohamate planimetry methods. The estimated age of each measurement data was calculated by evaluating the relationship between chronological age and gender.

Results: In male individuals, it was determined that there is a statistically significant difference between the real age and the chronological age estimates obtained using the Greulich-Pyle and Tanner-Whitehouse 2 methods, and there is no statistically significant difference between the real age and the chrono- 
logical age estimates estimated by the Capitohamate Planimetry method. It was determined by the Bland-Altman method that the estimates obtained by the Capitohamate Planimetry method were 5.1 units lower than the actual ages, and that the estimates obtained by the Tanner Whitehouse 2 method were 1.7 units higher than the actual ages. In female subjects, age estimates obtained by both Greulich Pyle and Tanner Whitehouse 2 methods were found to be 1.4 and 0.5 units lower than the actual ages, respectively, and there was no deviation in the Capitohamate Planimetry method.

Conclusion: According to the findings, it was concluded that the Capitohamate Planimetry method can be used reliably and with high accuracy for chronological age determination of children, and is an alternative estimation method to Greulich-Pyle and Tanner Whitehouse 2 atlases in the literature.

Keywords: capitohamate; greliuch-pyle; tanner-whitehouse; pediatric; radiology

\section{0-73}

\section{Standard values of wrist active range of motion}

Çolak I

Department of Orthopaedics and Traumatology, Kartal Dr. Lütfi Kırdar City Hospital, Istanbul, Turkey

Objective: Assessment of range of motion is an important physical examination step. It is frequently used in diagnosis, treatment and follow-up examination however, studies including our country's population are limited. The aim of this study is to evaluate the active range of motion of the wrist in healthy young adults.

Methods: Volunteer healthcare students, who did internship at Dr Lütfi Kırdar Kartal Training and Research Hospital between 2016 and 2018 were included in this study. Individuals who had previous upper extremity trauma or surgery were excluded from the study. Active range of motion measurements of wrist flexion, extension, radial and ulnar deviation were performed with a universal goniometer in accordance with the criteria recommended by the American Association of Orthopedic Surgeons. Data analysis was performed with the SPSS v.16 program using descriptive statistical methods.

Results: 57 women and 43 men participated in the study. The mean age of the participants was 21.6 $\pm 2.7(18-30)$ years, and their body mass index was $21.7 \pm 2.7(16.5-29.7)$. Mean right wrist flexion range of motion was $88.9^{\circ} \pm 3.1$ left wrist range of motion was $87.9^{\circ} \pm 3.5$; right wrist extension range of motion was $74.5^{\circ} \pm 6.2$, left wrist extension range of motion was $75.4^{\circ} \pm 5.9$; right wrist radial deviation was $27.1^{\circ} \pm 6$ left wrist radial deviation was $30.5^{\circ} \pm 6.3$; and right wrist ulnar deviation was $41.9^{\circ} \pm 9.2$ and left wrist ulnar deviation was $38.9^{\circ} \pm 7.6$.

Conclusion: The values of joint range of motion vary according to different concepts. The most widely used concepts are the American Association of Orthopedic Surgeons and Kendall. There is a limited number of studies conducted to determine the standard range of motion values for our country's population. The mean standard range of motion values may differ between countries therefore, studies with large sample sizes are needed for all joints

Keywords: goniometer, joint; range of motion, wrist

\section{0-74}

\section{The anatomy of the bipedal locomotion}

Ilgaz HB

Department of Anatomy, Faculty of Medicine, Hacettepe University, Ankara, Turkey

Objective: The walking style of the human species (homo sapiens), which began to evolve millions of years ago, has influenced survival behavior. In the process of moving from walking on all fours to standing on two feet, some changes have been observed in the body of our species, especially in the extremities. It is aimed to summarize these changes and to reveal their functional background.

Methods: Literature review was performed using the keywords "bipedalism and anatomy" in PubMed and Google Scholar databases.

Results: There are numerous theories related to the evolution of bipedal walking like energy theory, savanna-based theory, postural feeding hypothesis, threat theory, thermoregulatory theory, provisioning theory, etc. Data are available that support and do not support each theory. Although all these theories allow us to debate the cause of this evolutionary process, the results are actually quite clear. The center of the gravity in bipedal humans is changed, they have curved spine, weight is now transferred to the lower extremity by riding on the hip, the anteversion angle of the hip joint is increased, upper limb shortened, lower limb elongated compared to early primates, the shoulders have become lower and wider, the rib cage is enlarged, the hands are transformed so that they can perform opposition, the thumb is elongated, the human musculus flexor pollicis longus muscle is developed, the big toe has lost its ability to grip, the achilles tendon is elongated, the femurs are farther apart in the proximal than in the distal and are inclined towards the midline to keep the knees close together, the foramen magnum is placed more anterior and ventral, the gluteal muscles have developed to allow standing while passive, the angle of the valgus in the knee, feet with a higher transverse arch, especially hip and shoulder joints developed well and have become relatively larger articular faces. This evolutionary process did not occur in order to achieve the most perfect, bringing with it the disadvantages such as lower back and back pain and abdominal hernias more often than in early primates.

Conclusion: Evolutionary changes that have occurred over very long historical periods as a result of different mechanisms 
have revealed the current walking style of the human species. Anatomical changes brought about by this walking style are important for learning the functional anatomy of the extremities.

Keywords: evolution, bipedalism, quadrupedalism, comparative anatomy

\section{0-75}

Characterization of changes in the motor innervation of somatic muscles in a novel animal model created by viral mediated gene transfer

Polat Çorumlu E ${ }^{1}$, İlhan ÇF ${ }^{1}$, Büyükgüdük $\dot{I}^{1}$, Kapkaç HA², Arslanyolu $\mathrm{M}^{2}$, Ulupinar $\mathrm{E}^{1,3}$

'Interdisciplinary Neuroscience Department, Health Sciences Institute, Eskişehir Osmangazi University, Eskişehir, Turkey; '2Department of Molecular Biology, Faculty of Sciences, Eskişehir Technical University, Eskişehir, Turkey; ${ }^{3}$ Department of Anatomy, Faculty of Medicine, Eskişehir Osmangazi University, Eskişehir, Turkey

Objective: In motor neuron diseases, functions occurring automatically, including protection of respiration and body posture, become more energy-consuming due to weakness in the diaphragm, intercostal muscles and anti-gravity muscles. Serious losses in the motor units of swallowing and respiratory muscles cause inspiratory power, respiratory fatigue, hypercapnia and hypoxemia. Aim of this study to examine microscopic alterations in the diaphragm and triceps surae muscles and cervical-lumbar spinal motor neurons in an animal model created by using viral vector-mediated gene transfer in rats.

Methods: Viral vectors (serotype AAV2-9, 1.77x1013 vg/kg) designed to increase the expression of TDP-43 protein labeled with green fluorescent protein (GFP) were injected intravenously in 4 week-old Sprague-Dawley rats. Control animals were injected with SF (n=5/each group). The efficiency of transduction was evaluated by measuring changes in protein level by ELISA test. Following intracardiac perfusion two weeks after injection, sections taken from diaphragm, triceps surae and spinal cord tissues were stained with hematoxylineosin and methylene blue, respectively. Presynaptic axon terminals ending in muscle tissue were analyzed by synaptophysin immunohistochemistry. The number and soma diameters of alpha-motor neurons in the anterior horns of the cervical and lumbar spinal cord segments and the ratio of muscle fibers in the diaphragm and triceps surae to the septal areas consisting of connective tissue were compared with morphometric analysis.

Results: In comparative analyses of the groups, alpha-motor neuron counts in both the cervical and lumbar regions were significantly $(\mathrm{p}<0.001)$ lower in animals transduced with TDP43. A significant decrease was observed in the ratio of muscle fibers to septal areas in the diaphragm and leg muscle sections in parallel with the deficiencies observed in the respiratory and skill-required motor functions of the experimental animals. Similarly, in the experimental groups, a significant decrease was observed in the axon terminals and endplate structures that innervate the somatic muscle fibers.

Conclusion: In the generated animal model, increase in the TDP-43 protein level leads to progressive loss of lower motor neurons, resulting in phenotypic changes similar to clinical symptoms observed in ALS patients. Since the damage in cervical spinal cord segments precedes the lumbar segments, disturbances in the innervation of diaphragm was observed more prominently than those of hindlimbs. This technique is promising in future for treatment of motor neuron diseases as it can also be used to transfer genes encoding protective molecules to damaged muscles or neurons.

This study is supported by TÜBİTAK-Grant \#116S408.

Keywords: diaphragm, triceps surae muscle, viral vectors, TDP-43, synaptophysin

\section{0-76}

Analysis of the effects of hypothalamic pituitary adrenal axis activity and pain in menstrual cycle on visual and auditory reaction times and women's health

Şenol D ${ }^{1}$, Uçar C ${ }^{2}$, Altınoğlu $M^{3}$, Özdemir B ${ }^{3}$, Bilge D ${ }^{3}$, Özbağ D $\mathrm{D}^{3}, \mathrm{Y}_{1} \mathrm{ld}_{\mathrm{d}} \mathrm{S} \mathrm{S}^{4}$

'Department of Anatomy, Faculty of Medicine, Düzce University, Düzce, Turkey; '2Department of Physiology, Faculty of Medicine, Kahramanmaraş Sütçü Imam University, Kahramanmaraş, Turkey; ${ }^{3}$ Department of Anatomy, Faculty of Medicine, Inönü University, Malatya, Turkey; ${ }^{4}$ Department of Physiology, Faculty of Medicine, Inönü University, Malatya, Turkey.

Objective: The aim of this study was to analyse the effects of hypothalamic pituitary adrenal (HPA) axis activity and pain in menses $(\mathrm{M})$, periovulatory $(\mathrm{PO})$, luteal phase (LP) and premenstrual (PM) phases of menstrual cycle (MC) on visual reaction time (VRT) and auditory reaction time (ART) and to evaluate these in terms of women's health.

Methods: 73 women between the ages of 20 and 25 participated in this study voluntarily. In order to find the cortisol levels, saliva samples were taken from the participants in MC phases and the samples were analysed with Enzyme-Linked Immunosorbent Assay (ELISA). Cortisol analysis was supported with State-Trait Anxiety Inventory (STAI-I). Pain evaluation was made with Visual Analogue Scale (VAS). Reaction timer was used for VRT and ART measurement. In MC phases, statistical analysis of cortisol, STAI-I VAS, VRT and ART variables were made with Kruskal Wallis H test. Mann Whitney $U$ test was applied on the data as a post hoc test. Spearman Rho correlation was applied on the data to find out how VRT and ART scores changed according to cortisol, STAI-I and VAS values. IBM SPSS Statistics 22.0 for Windows program was used in analyses. 
Results: According to the Kruskal Wallis $\mathrm{H}$ test conducted, it was found that VAS, VRT and ART variables showed statistically significant difference in different MC phases $(\mathrm{p}<0.05)$. According to the post hoc test carried out, it was found that the difference occurred due to the menses phase. According to the correlation analysis result, a positive weak correlation was found between VAS scores in the menstrual phase and VRT and ART scores.

Conclusion: Stress causes obvious changes in emotional state and hormonal secretion. These include the activation of the HPA axis, which causes a high level of glucocorticoid secretion from the adrenal cortex. In our study, it was found that VRT and ART scores were not affected by the stress in MC, while pain had a negative effect on reaction time.

Keywords: menstrual cycle, HPA axis, stress, cortisol, reaction, women's health

\section{0-77}

Effects of astaxanthin on autophagy in rats with renal ischemia reperfusion injury

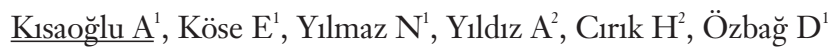
'Department of Anatomy, Faculty of Medicine, Inönü University, Malatya, Turkey; ${ }^{2}$ Department of Histology and Embryology, Faculty of Medicine, Inönü University, Malatya, Turkey

Objective: Renal damage that occurs depending on ischemia and reperfusion is an important common problem in society and non-treated yet. Astaxanthin is a pigment known for having high antioxidant property. Autophagy, one of the cellular death pathways, is expressed as a cellular process that plays a role in diseases such as cancer, metabolic and neurodegenerative disorders. The aim of this study is to investigate the effect of astaxanthin on autophagy in rats with renal ischemia reperfusion injury.

Methods: 40 male Sprague-Dawley rats were used in this study. The rats were divided into 5 groups; Group 1 (Sham), Group 2 (I/R), Group 3 (I/R+Atx 5 mg/kg), Group 4 (I/R+Atx 10 mg/kg) and Group 5 (I/R+Atx $25 \mathrm{mg} / \mathrm{kg})$. The rats in groups 1 and 2 were given astaxanthin solvent (olive oil) by oral gavage for 7 days. The rats in groups 3, 4 and 5 were given different doses of astaxanthin dissolved in olive oil by oral gavage during 7 days. At the end of 7 th day, rats in all groups were performed bilateral ischemia for 45 minutes then reperfusion for 24 hours. Beclin-1, LC3, p62 levels in renal tissues were detected by immunohistochemistry method. Increased Beclin-1 and LC3 level is associated with presence of autophagy. Decreased p62 level is associated with an activated autophagy pathway.

Results: Necrotic changes and dilatation were observed in the tubules in both cortical and medullary areas in the I/R group. Another prominent finding in this group was inflammatory cell infiltration that was locally and diffuse in the interstitial tissue. It was detected that the histopathological findings observed in the
I / R group decreased significantly in the Atx $10 \mathrm{mg} / \mathrm{kg}$ and Atx $25 \mathrm{mg} / \mathrm{kg}$ groups. LC3 and Beclin-1 immunoreactivity were detected the highest in Atx $25 \mathrm{mg} / \mathrm{kg}$ group when compared with sham, Atx $5 \mathrm{mg} / \mathrm{kg}$ and Atx $10 \mathrm{mg} / \mathrm{kg}$ groups. p62 immunoreactivity was detected the lowest in the Atx $25 \mathrm{mg} / \mathrm{kg}$ group.

Conclusion: In the experimental study, it was determined that astaxanthin administration induced autophagy. Astaxanthin protected the cells against damage by autophagy mechanism.

Keywords: renal, ischemia/reperfusion, astaxanthin, autophagy

\section{0-78}

Does Cornus Mas L have anti-tumor effect on the brain? Yılmaz S ${ }^{1}$ Tokpınar A ${ }^{1}$, Ateş $S^{1}$, Oflamaz A², Doğanyiğit $Z^{2}$ 'Department of Anatomy, Faculty of Medicine, Yozgat Bozok University, Yozgat, Turkey; 'Department of Histology and Embryology, Faculty of Medicine, Yozgat Bozok University, Yozgat, Turkey

Objective: All over the world, cancer cases are constantly increasing and studies of anticancer of herbs in complementary medicine continue. In our study, the antitumoral effect of Cornus Mas L plant on the brain in mice with liquid tumors is investigated.

Methods: Eight-week-old Balb / C strain mice with an average weight of 25-30 g were used in the study. After Ehrlich Acid Tumor (EAT) cells were removed from -80 degrees and melted at room temperature, they were injected into the peritoneum area between the left hind leg of the mouse and the abdomen, and our stock group was formed. We have three groups in the study. The negative control group was the group that did not develop cancer, in the positive control group, 1X106 EAT cells were injected into the peritoneum area on day 0. Cornus Mas L $200 \mathrm{mg} / \mathrm{kg}$ group, cancer-induced group $200 \mathrm{mg} / \mathrm{kg}$ Cornus Mas L plant extract was given daily. On the 9th day, animals were anesthetized and sacrificed. Brain tissues extracted from experimental groups were evaluated histopathologically by using hematoxylin and eosin staining method. In the immunohistochemical analysis, the immunoreactivity of Factor VIII protein was determined in the brain tissue using the Avidin-Biotin peroxidase method.

Results: Initially, animal weight was $28 \mathrm{~g}$ in the negative control group, $29.1 \mathrm{~g}$ in the positive control group and $30 \mathrm{~g}$ in the 200 $\mathrm{mg} / \mathrm{kg}$ Cornus Mas L group. At the end of the 9th day, animal weights were $33.5 \mathrm{~g}$ in the negative control group, $40 \mathrm{~g}$ in the positive control group, and $37 \mathrm{~g}$ in the $200 \mathrm{mg} / \mathrm{kg}$ Cornus Mas $\mathrm{L}$ group. There is a statistical difference between the groups $(\mathrm{p}<0.001)$. When the positive control group and the $200 \mathrm{mg}$ Cornus Mas L group were examined histopathologically, it was observed that there was no significant difference between the groups. Factor VIII expression in the brain tissue cortex was not found to be significantly different between the experimental groups, while it was significantly decreased in the group treated with $200 \mathrm{mg}$ Cornus Mas L in the hippocampus ( $<<0.05)$. 
Conclusion: Our study showed that cranberry plants have an antitumoral effect on the formation of acid tumors by EAT cells. Keywords: brain, tumor, cornus mas L, cell

\section{0-79}

\section{Can Whitnall's tubercle be localized using the landmarks on face?}

Korkmaz A ${ }^{1}$ Salman $\mathrm{N}^{1}$, Uz A A $^{1,2}$

'Anatomy Department, School of Medicine, Ankara University, Ankara, Turkey; ${ }^{2}$ Department of Neuroscience, Health Sciences Institute, Ankara University, Ankara, Turkey

Objective: A numerous important structures have attachments with Withnall's tubercle, located deep in the lateral canthus and orbit wall. In our study, we aimed to define the location of Whitnall's tubercle by using the superficial bone landmarks.

Methods: 44 orbits were divided into two groups according to the prominence or absence of the Whitnall's tubercle. Craniums were located on the Frankfort horizontal plane and Whitnall's tubercles were marked. The distances of the Whitnall's tubercle to the frontozygomatic suture, the marginal tubercle, the anterior point of zygomatic arch, and the lateral orbit wall were measured. The clock positions of Whitnall's tubercle according to the marginal tubercle and the anterior point of zygomatic arch were determined.

Results: The distances of the Whitnall's tubercle to the marginal tubercle, the anterior point of zygomatic arch, the lateral orbital wall, and the frontozygomatic suture were measured as $9.92 \pm 1.65 \mathrm{~mm}, 16.48 \pm 1.86 \mathrm{~mm}, 2.32 \pm 0.53 \mathrm{~mm}$ ve $9.66 \pm 1.44$ $\mathrm{mm}$, respectively. Whitnall's tubercles were positioned at 8 (13 orbits) and 9 (9 orbits) o'clock positions at left side; and at 4 (15 orbits), 3 (6 orbits), and 2 (1 orbit) o'clock positions according to the marginal tubercle. Whitnall's tubercles were at 10 o'clock position at left side; at 2 (20 orbits) and 1 ( 2 orbits) positions at right side according to the anterior point of zygomatic arch.

Conclusion: The marginal tubercle and the anterior point of zygomatic arch can be used as bony landmarks in eyelid and orbit surgery in order to prevent the damage of the soft tissue relevant to Whitnal's tubercle.

Keywords: Whitnall's tubercle, orbit, lateral canthus, marginal tubercle, zygomatic arch

\section{0-80}

Investigation of the morphometry of the coracoid process Ayazoğlu M, Özdemir B, Öztürk K, Kastamoni Y

Department of Anatomy, Faculty of Medicine, Süleyman Demirel University, Isparta, Turkey

Objective: Examination of morphometric properties of coracoid process due to its clinical importance in pathologies that may occur around the shoulder.
Methods: Our study was carried out on 18 scapulas ( 9 right, 9 left) in situated Suleyman Demirel University Faculty of Medicine Department of Anatomy Laboratory. Scapulas with broken or defective coracoid processes were not included in the study. First, three types of coracoid process were determined according to the shape of the region between coracoid process and glenoid cavity: Type I; round, Type II; square and Type III; hook. The length, base thickness, tip thickness and coracoacromial distance of the coracoid process were measured with a digital caliper. The lines passing through the end point of the coracoid process and parallel to the midline of the coracoid process and passing through the supraglenoid tubercle and through the extreme point of the inferior angle were determined. The angle between the lateral midline of the scapula and the coracoid process was determined as the angle passing between these two lines. The angle was measured using the Image J program. Data was analyzed with SPSS 20 for Windows.

Results: In scapulas, the region between coracoid process and glenoid cavity was found at a rate of Type I $55.56 \%$, Type II $22.22 \%$, Type III $22.22 \%$. The average length of coracoid process was $40.08 \pm 5.61 \mathrm{~mm}$, average base thickness $12.56 \pm 2.65$ $\mathrm{mm}$, average tip thickness $7.94 \pm 1.16 \mathrm{~mm}$ and mean coracoacromial distance $32.06 \pm 6.31 \mathrm{~mm}$ and the average angle between the lateral midline of the scapula and the coracoid process was $126.62 \pm 8.68$ degrees. In the comparison between the right and left sides, a statistically significant difference was found in the parameters of the length of the coracoid process $(p=0.018)$ and the angle between the lateral midline of the scapula and the coracoid process $(\mathrm{p}=0.048)$.

Conclusion: It is known that the coracoid process, which is longer than normal, plays a role in the etiology of impingement syndrome. Although there are studies showing that coracoid process morphometry is a key structure in shoulder surgery and shoulder pathologies, there are limited studies evaluating coracoid process separately from the scapula whole. In our study, unlike other studies, the angle between the lateral midline of the scapula and the coracoid process was measured and a significant difference was found in the comparison between the sides. For this reason, we think that our study will contribute to the limited literature on coracoid process morphometry and offer clinicians a different perspective.

Keywords: scapula, impingement syndrome, coracoacromial distance, glenoid cavity

\section{0-81}

\section{Anthropometric evaluation of the humerus}

Akça Andı Z, Özcan BN, Uzun A, Emirzeoğlu M

Department of Anatomy, Faculty of Medicine, Ondokuz Mayıs University, Samsun, Turkey

Objective: The humerus is the longest and largest bone of the upper limb, and determining the humerus length from seg- 
mental and morphometric measurements is important for forensic anthropology in terms of sex and race determination. The aim of the present study was to determine the morphometric and segmental measurements of humerus in our population and to calculate the mean standard value and also compare the findings with other populations to assist in forensic and archeological cases.

Methods: A total of 52 humeri (27 right, 25 left) belonging to the bone collection of the Anatomy Department were included in the study. Sixteen measurements (Length of the humerus, the transverse and vertical diameter of the humeral head, the diameter and circumference of the humeral body, surgical neck circumference, humeral body circumference, humeral body diameter etc.) were taken from different parts of the humerus. Measurements were taken using tape measure and $0.1 \mathrm{~mm}$ precision digital vernier caliper. Mean values and standard deviation values of the obtained data were calculated.

Results: According to the data obtained from the study; average values for the length of the humerus was $309.56 \pm 24.43 \mathrm{~mm}$ for the right and $309.82 \pm 19.05 \mathrm{~mm}$ for the left side; surgical neck circumference was $7.53 \pm 0.98$ and $7.70 \pm 0.81 \mathrm{~cm}$, respectively. For the length and width of intertubercular sulcus ( $\mathrm{p}=0.034 ; \mathrm{p}=0,038)$, the distance between the most distal point of olecranon fossa and trochlea of humerus $(\mathrm{p}=0.041)$ and the distance between the proximal edge of the olecranon fossa, and the most proximal point of trochlea of humerus $(\mathrm{p}=0.021)$ there were statistically significant differences between right and left sides. No significant difference was found between the left and right sides in other parameters.

Conclusion: The present study findings help in orthopaedic surgery practice, for the treatment of proximal and distal humerus fractures and for their reconstruction. Also, the measurement values to be obtained from humerus are also important in terms of race, gender and age estimation. We think that the average values obtained as a result will contribute to orthopedic surgery and forensic science.

Keywords: humerus, anthropometry, morphometry

\section{0-82}

\section{Clinical evaluation of morphometric characteristics of the lamina cribrosa}

Açıkgöz AK, Erdem H

Department of Anatomy, Medical Faculty, Çukurova University, Adana, Turkey

Objective: Lamina cribrosa, a thin and fragile lamellar bony structure, is in very close relationship with fossa cranii anterior. Severe trauma might cause many complications like fracture, cerebrospinal fluid flow, lobus frontalis injury, meningitis and n. olfactorius injury. Therefore, the aim of this study was to determine the morphometric features of lamina cribrosa.
Methods: Measurements were performed with a digital sliding caliper accurately to $0.1 \mathrm{~mm}$, on 23 craniums that belonged to the Cukurova University Medical Faculty, Department of Anatomy. Fractured or deformed skulls were excluded. Statistical analysis was performed with SPSS 20.0.

Results: The mean length of the lamina cribrosa were found as $22.71 \pm 1.88 \mathrm{~mm}$ and $22.30 \pm 1.84 \mathrm{~mm}$, the lamina cribrosa width were found as $4.23 \pm 1.11 \mathrm{~mm}$ and $4.36 \pm 1.52 \mathrm{~mm}$ and crista galli depth were found as $10.92 \pm 3.14 \mathrm{~mm}$ and $10.76 \pm 2.85 \mathrm{~mm}$ for right and left sides, respectively. Crista galli width was $3.71 \pm 1.11 \mathrm{~mm}$; and total lamina cribrosa width was $12.32 \pm 2.52$ $\mathrm{mm}$. No statistically significant differences were observed between right and left side measurements $(p>0.05)$.

Conclusion: Although many conservative techniques have been developed, the identification of the apertura sinus frontalis and risk of restenosis is still two problems of endonasal surgery. Knowledge of the anatomy of this region is too crucial to be able to overcome these problems. When the localization of the apertura sinus frontalis can be determined drilling can be performed lateromedially while in cases that cannot be determined it can be performed mediolaterally under the sinus floor and in front of the cribriform plate to prevent cerebrospinal fluid leak. For this reason, It is essential to have knowledge of the anatomy and variations in this area. We think our results can guide the interventions around the lamina cribrosa and surgeons may have benefited from our results.

Keywords: crista galli; endonasal surgery; fossa cranii anterior; lamina cribrosa

\section{0-83}

\section{The morphologic and morphometric features of the triquetrum}

$\underline{\text { Balcı A }}{ }^{1}$, Y1ldiran $\mathrm{G}^{2}$, Kendir $\mathrm{S}^{1}$, Karahan $\mathrm{ST}^{1}$, Apaydın $\mathrm{N}^{1,3,4}$

'Department of Anatomy, School of Medicine, Ankara University, Ankara, Turkey; ' ${ }^{2}$ linic of Hand Surgery, Konya City Hospital, Konya, Turkey; ${ }^{3}$ Department of Multidisciplinary Neuroscience, Institute of Health Sciences, Ankara University, Ankara, Turkey; ${ }^{4}$ Brain Research Center (AU-BAUM), Ankara University, Ankara, Turkey

Objective: Fractures of the triquetrum are the second most common isolated carpal bone fracture after fractures of scaphoid. However, there is limited data on the morphology and morphometry of triquetrum in literature. The aim of this study therefore is to describe the morphology of triquetrum using anatomical landmarks, to evaluate its morphometric features and to determine its vascular entry points.

Methods: The morphological and morphometrical features of 43 adult triquetral bones ( 21 left,22 right) were determined by measuring the length, width and thickness of the bone. Number and location of nutrient foramina, which are wider than $0.5 \mathrm{~mm}$ in diameter, were assessed. ANOVA test was used for comparing the number of foramina. $\mathrm{P}$ value $<0.05$ was considered as statistically significant. 
Results: The length of triquetrum was defined as the distance between distal pole and dorsal edge of interarticular ridge. The mean length of triquetrum was $17.86 \mathrm{~mm}$ (min: 15.04-max: $22.13 \mathrm{~mm}$ ). The width was defined as the distance between palmar edge of interarticular ridge and dorsal border of articular surface of hamate. Accordingly, the mean width was $13.00 \mathrm{~mm}$ (min: 11.00-max: $15.85 \mathrm{~mm}$ ). The thickness was measured from the midpoint of the height of the bone and was a mean of $11.30 \mathrm{~mm}$ (min: 8.98-max: $14.74 \mathrm{~mm}$ ). All of the bones had articular face with pisiform on the distal-medial side of their palmar surface. This face was oval-shaped in 20 bones, roundshaped in 3 bones and amorphous(neither round or oval) in 20 bones. Borders of articular face with pisiform was prominent in 23 bones. All of bones had interarticular ridge. Mean length of interarticular ridge was $7.1 \mathrm{~mm}$ (min: 5.50-max: $8.67 \mathrm{~mm}$ ). Mean number of nutrient foramen on the bones was 7 (min: 1-max: 18). The data was normally distributed according to Kolmogorov-Smirnov test. The number of nutrient foramina located on the dorsal aspect of the bone was 4.58 \pm 3.17 (min: $0-\max : 14$ ), between pisiform and lunatum 2.05 \pm 1.40 ( $\mathrm{min}$ : $0-\max : 6$ ) and on the other surfaces $0.44 \pm 0.98$. The difference between these 3 groups was statistically significant $(\mathrm{p}<0.05)$. The dorsal tubercle was prominent on $74 \%$ of the bones.

Conclusion: Triquetrum is a pyramidal shaped bone with a base facing lunate. The location of nutrient foramina indicates that the blood supply of the bone is mainly provided by the vessels coming from its dorsal side. The predominance of the nutrient foramina on its dorsal aspect makes the bone weaker in the dorsal region and it supports the fact that the fractures are more common in this region. Besides that, the dorsal aspect is rich in blood supply and that can be the reason why the avascular necrosis are less common in triquetral fractures.

Keywords: triquetrum, morphometry, morphology, carpal bones

\section{0-84}

Neuroinflammatory changes in a novel motor neuron disease model created by viral vector- mediated gene transfer method in rats

Illhan $\mathrm{CF}^{1}$, Polat Çorumlu E ${ }^{1}$, Büyükgüdük $\dot{\mathrm{I}}^{1}$, Kapkaç $\mathrm{HA}^{2}$, Arslanyolu $\mathrm{M}^{2}$, Ulupinar $\mathrm{E}^{1,3}$

'Department of Interdisciplinary Neuroscience, Health Sciences Institute, Eskişehir Osmangazi University, Eskişehir, Turkey; '2Department of Molecular Biology, Faculty of Sciences, Eskişehir Technical University, Eskișehir, Turkey; ${ }^{3}$ Department of Anatomy, Faculty of Medicine, Eskişehir Osmangazi University, Eskişehir, Turkey

Objective: In recent years, TDP-43 (transactivate response DNA-binding protein-43) proteinopathy has emerged as a common mechanism in neurodegenerative diseases that cause progressive loss in motor as well as frontal and temporal lobe cortical neurons. Interestingly, it has been shown that changes in the expression level of this protein can both decrease and increase neuroinflammation, especially in diseases with dementia, such as Alzheimer's Disease. In this study, it was aimed to investigate the morphological changes that occur in cell groups that mediate neuroinflammation in two different animal models created by inducing changes in native or mutant TDP-43 level.

Methods: In order to perform the transfection specific to motor neurons; genes that will enable the expression of native or deleted forms of the "nuclear localization signal" (NLS) TDP-43 protein are packaged into a viral vector capsid (serotype AAV2 / 9), under the control of a special promoter (UCHL1). While the virus particles $(1.77 \times 1012 \mathrm{vg} / \mathrm{kg}, \mathrm{n}=5 /$ each group) were injected through the tail vein to the experimental groups on the postnatal day 30, the control animals received the same amount of SF. After 4 weeks of the systemic injection, animals were perfused by intracardiac route and sections taken from the motor cortex region were analyzed by systematic randomized sampling method which were stained immunohistochemically using Ibal and GFAP, antibodies specific to microglia and astrocyte cells.

Results: Statistical analysis showed that the number of Iba (+) microglia per unit area in the motor cortex of both native and NLS deleted groups increased significantly $(\mathrm{p}<0.05$ and $\mathrm{p}<0.001$, respectively). Similarly, a significant increase was observed in the immunoreactivity levels in astrocytic cells expressing GFAP in both groups compared to the control group.

Conclusion: Our data show that different expression of TDP43 protein in cortical cells of rats causes neuroinflammatory changes observed in the form of microglial activation and astrogliosis. These animal models developed through viral vectors are important in elucidating the pathological changes and underlying cellular mechanisms that occur in neurodegenerative diseases. These models can also be used to test new therapeutic approaches.

This project is supported by TÜBİTAK (Project No: \# 116S408).

Keywords: microglia, astrocyte, viral vector, TDP-43, Iba, GFAP

\section{0-85}

The histopathological evaluations in different septorhinoplasty techniques and poly-p-dioxanone plate on nasal septum of rabbits

Uğurlu BN ${ }^{1}$, Doğan $\mathrm{DE}^{2}$, Çelik $\mathrm{H}^{3}$, Aslan Felek $\mathrm{S}^{3}$, Aktar Uğurlu $\mathrm{G}^{4}$, Zeybek ND²

'Department of Otorhinolaryngology, Akdağmadeni State Hospital, Yozgat, Turkey; ' ${ }^{2}$ epartment of Histology and Embryology, Hacettepe University Medical Faculty, Ankara, Turkey; ${ }^{3}$ Department of Otorhinolaryngology, Ankara Training and Research Hospital, Ankara, Turkey; ${ }^{4}$ Department of Otorhinolaryngology, Hitit University Medical Faculty, Çorum, Turkey 
Objective: Nasal septum plays a key role in midfacial growth. The techniques that can be applied to the septum in pediatric septorhinoplasty affect this growth. We aimed to investigate the histopathological changes seen with different techniques of septorhinoplasty and the use of poly-p-dioxanone (PDS) plates in growing noses.

Methods: Thirty-three, eight-weeks-old rabbits were included in the study and divided into 5 groups. Rabbits in group 1 were observed to grow without any intervention. In group 2, the nasal dorsum of the rabbits was opened and the mucosa was bilaterally elevated. In group 3, in addition to the procedures in group 2, limited cartilage resection was performed while preserving the nasal dorsum. In group 4, in addition to group 3, the excised cartilage was crushed with a cartilage crusher and crushed cartilage was placed back between the mucoperichondrium. In group 5, the crushed cartilage was placed back between the mucoperichondrium as a composite with a PDS plate. All rabbits were observed until 20 weeks of age and at the end of the 20th week, they were sacrificed and their nasal septums were removed. Specimens were decalcified and fixed in $10 \%$ buffered formalin, then dehydrated through graded alcohols and embedded in paraffin. Sections of five $\mu \mathrm{m}$ thick were cut and stained with hematoxylin-eosin ( $\mathrm{H} \& \mathrm{E})$. The specimens were examined using a light microscope and evaluated according to changes in the cartilage viability and changes of tissue including fibrosis, inflammation, vascularization, metaplastic bone formation, fragmentation. Kruskal Wallis test used for statistical analysis. The results were considered statistically significant for $\mathrm{p}<0.05$.

Results: In the group 2, there were prominent inflammation and fibrosis more than other groups. The group 5 showed distinct fragmentation and fibrosis. There was a statistically significant difference between the group 5 and group 1, 2 for fragmentation $(p<0.05)$. All of the groups showed peripheral chondrocyte proliferation and none of them showed metaplastic bone formation.

Conclusion: In pediatric septal surgeries, limited cartilage resections can be safely performed, while preserving septodorsal cartilage. Although positive results have been reported regarding the use of PDS plate in adults, its use in growing noses may affect maxillofacial growth negatively.

Keywords: nasal septum, septorhinoplasty techniques, PDS, cartilage, rabbit

\section{0-86}

The effect of a calcium channel blocker, isradipine, on calbindin blood levels and calbindin positive neurons in the striatum of Parkinsonian rats lesioned with 6-OHDA ${\text { Boracı } \mathrm{H}^{1}}$, Kirazlı Ö$^{1}$, Gülhan $\mathrm{R}^{2}$, Verimli U' ${ }^{1}$, Şehirli ÜS 'Department of Anatomy, School of Medicine, Marmara University, Istanbul, Turkey; ${ }^{2}$ Department of Medical Pharmacology, School of Medicine, Marmara University, Istanbul, Turkey

Objective: Parkinson's disease (PD) is a slowly progressing neurodegenerative disease, characterized by the loss of dopaminergic neurons in the substantia nigra pars compacta. In pathology, the imbalance of the intracellular calcium level is important and may appear as a consequence of abnormality related with calcium buffering mechanisms. Calcium binding proteins, like calbindin, play an important role in the buffering mechanisms and regulate L-type voltage dependent calcium channel alpha 1D subunit (CaV1.3) in the brain. CaV1.3 has a role in dopaminergic neurons that are selectively susceptible to neurodegeneration. In this study, we examined the effect of a calcium channel blocker, isradipine, on the blood levels of calbindin and calbindin positive neurons in the striatum.

Methods: 6-OHDA was stereotaxically injected into the medial forebrain bundle. Control and lesioned groups were treated with isradipine $(2 \mathrm{mg} / \mathrm{kg}$ ) or dimethyl sulfoxide (DMSO) subcutaneously for 4 weeks (Schuster, 2009). On the 21st day, the apomorphine rotation test $(0.05 \mathrm{mg} / \mathrm{kg})$ was carried out, at the end of 4th week, the rats were sacrificed. The enzyme-linked immunoadsorbent assay was used to measure the blood levels of calbindin-28K. Brains were immunohistochemically stained by thyrosine-hdroxylase and calbindin. The sections were examined under fluorescence and light microscope. The results were analyzed by two-way ANOVA followed by a post-hoc Tukey test in Graph-Pad PRISM 6.0 (GraphPad Software Inc., San Diego, CA, USA) program.

Results: The number of rotations (21st day) was significantly lower ( $\mathrm{p}=0.0076$ ) in Parkinsonian isradipine group compared to Parkinsonian DMSO group. The calbindin blood levels in Parkinsonian isradipine group was higher compared to Parkinsonian DMSO group $(\mathrm{p}<0.05)$. The increase in the calbindin blood levels of Parkinsonian isradipine group was also significant compared to the control group with no lesion $(\mathrm{p}<0.05)$. On the other hand, there was no significant difference in the number of calbindin positive neurons in the striatum of all groups.

Conclusion: As a conclusion, the mechanism of action of isradipin upsurge the calbindin levels which in turn may improve the pathology of PD. Hence, the calbindin may be highlighted as a biomarker for the clinical process of Parkinson's disease.

Keywords: Parkinson's disease, striatum, isradipine, calbindin

\section{0-87}

Effects of syringic acid on iNOS expression and nitrate/nitrite levels in 6-OHDA-induced experimental Parkinson model

$\underline{\text { Güzelad } \ddot{O}^{1}}$, Özkan A², Parlak $\mathrm{H}^{2}$, Sinen $\mathrm{O}^{2}, \mathrm{Afşar} \mathrm{E}^{3}$, Öğüt $E^{4}$, Yıldırım FB ${ }^{1}$, Bülbül $M^{2}$, Ağar A ${ }^{2}$, Aslan $M^{3}$

'Department of Anatomy, Faculty of Medicine, Akdeniz University, Antalya, Turkey; ' 2 Department of Physiology, Faculty of Medicine, Akdeniz University, Antalya, Turkey; ${ }^{3}$ Department of Biochemistry, Faculty of Medicine, Akdeniz University, Antalya, Turkey; " Department of Anatomy, Faculty of Medicine, Bahçeşehir University, Istanbul, Turkey 
Objective: 6-Hydroxydopamine (6-OHDA) is a widely used chemical to create an experimental Parkinson model on rats. There was no study showing the protective effect of syringic acid (SA) in the substantia nigra on inducible nitric oxide synthase (iNOS) expression, nitrite and nitrate levels in Parkinson's disease. This study aimed to investigate the potential protective effect of SA on iNOS expression, nitrite and nitrate levels on the experimental Parkinson model induced by 6-OHDA.

Methods: The present study was performed in Rattus norvegicus Wistar albino male adult rats $(\mathrm{n}=75)$. The groups were determined as Sham (S), Parkinson (P), Syringic acid (SA), Syringic acid + Parkinson $(\mathrm{SA}+\mathrm{P})$ and Parkinson + Syringic acid $(\mathrm{P}+\mathrm{SA})$ groups. 6-OHDA was injected bilaterally into the medial forebrain band by stereotaxic surgery. The iNOS levels (ng/mg) in substantia nigra tissue samples obtained from the subjects were evaluated the ELISA (Elabscience, Catalog No: E-EL-R0520, Houston, Texas, USA), and the number of iNOS positive cells evaluated by immunofluorescence method. The nitrite and nitrate levels $(\mu M)$ were determined by the colorimetric kit (Cayman Chemical, Catalog \# 780001, Ann Arbor, MI, USA).

Results: In the Parkinson group, 6-OHDA significantly increased iNOS expression (ng/mg), nitrite and nitrate levels $(\mu \mathrm{M})$ compared to the Sham group. $(\mathrm{p}<0.001)$. The iNOS expression $(\mathrm{ng} / \mathrm{mg})$ nitrite and nitrate $(\mu \mathrm{M})$ levels were significantly decreased in the syringic acid-treated group when compared to the Parkinson group $(\mathrm{p}<0.001)$. In the immunofluorescence analysis, the number of iNOS positive cells significantly decreased in the SA group compared to the Parkinson group $(\mathrm{p}<0.05)$.

Conclusion: In conclusion, syringic acid significantly decreased nitrite and nitrate levels by inhibiting iNOS expression against 6-OHDA toxicity. This might be caused by reduced microglia activation through the anti-inflammatory effects of syringic acid. Therefore, syringic acid can be used as a potential therapeutic agent in the treatment of Parkinson's disease via its inhibitor effects over iNOS expression.

Keywords: Parkinson's disease, 6-Hydroxydopamine, syringic acid, iNOS, nitrite, nitrate

\section{0-88}

Results of experimental study of blood circulation in visceral branches of the abdominal aorta

\section{Babayeva R}

Department of Human Anatomy and Medical Terminology, Faculty of Medicine, Azerbaijan Medical University, Baku, Azerbaijan

Objective: Experimental study of blood circulation in certain visceral branches of abdominal aorta.

Methods: Some branches of the abdominal aorta (coeliac trunk, superior and inferior mesenteric arteries) in 20 rats.
Methods applied include I. Markov's silver plating, application of hematoxylin for detection of microcirculatory network and electron-microscope method. $0.05 \mathrm{mg}$ ergometrine maleate solution was used to cause vascular spasm. All experiments on animals were carried out in strict compliance with European Ethical Standards.

Results: Coeliac trunk, as well as superior and inferior mesenteric arteries are arteries of the muscular-elastic type. Their walls contain 20-40 layers of myocytes. The outer and inner elastic membranes are clearly defined. The adventitia layer is wide, with a large number of blood and lymphatic microvascular and nerve elements. The angle of branching of the coeliac trunk from the aorta is $\approx 300$, the angle of branching of the superior mesenteric artery is $\approx 320$, and the angle of branching of the inferior mesenteric artery is $\approx 240$. Significant dilatations (to diffusers) were observed along the length of all these vessels. In addition, the superior mesenteric artery forms an arch with a radius of $\pm 2.4 \mathrm{~cm}$. This anatomical position of the superior mesenteric artery allows all intestinal arteries to branch at an angle of $36 \pm 40$. The angle of the arc formed by the inferior mesenteric artery is relatively small $(4.0 \pm 1.0 \mathrm{~cm})$. The results of the study showed that the blood circulation in the coeliac trunk begins to increase 35-30 minutes after the introduction of 250 $\mathrm{ml}$ of milk to the rats through the probe and reaches the maximum level in 40 minutes. Blood circulation in the superior mesenteric artery begins to increase 5-7 minutes after the introduction of milk into the duodenum. The effect of ergometrine on blood circulation in the coeliac trunk and superior mesenteric artery was relatively noticeable. Visually, 5 minutes after intra-arterial injection of $0.05 \mathrm{mg}$ ergometrine maleate, a strong contraction of the stomach and intestines was detected. After 10 minutes, the contraction stopped. The size of the stomach is reduced, intestinal spasm was observed (contracted). Pulse pressure was not detected in the small-diameter arteries of the stomach and intestines, and blood circulation in these arteries stopped. The circulation in the coeliac trunk and superior mesenteric artery reduced by 1.5-2 times. After passing through the muscle "gates", the straight arteries divide into two arteries of different diameters, forming the main submucosal network of the gastrointestinal tract due to the large number of arterio-arterial anastomoses. In transverse sections, interaction of the elongated layer of the muscular coat with the arteries and arterioles, veins, venules, lymphatic vessels and capillaries passing through it is more pronounced.

Conclusion: Coeliac trunk, superior and inferior mesenteric arteries are arteries of the muscular-elastic type. Visually, a strong contraction of the stomach and intestines is observed 5 minutes after the ergometer maleate solution is injected into the artery. After 10 minutes, the contraction stops, the stomach shrinks, and spasms are observed in the intestines. In small diameter arteries of the stomach and intestines, pulse pressure is not detected, and blood circulation in these arteries stops

Keywords: coeliac artery, mesenteric artery, ergometrine 


\section{0-89}

Morphologic evaluation of dorsal surface of sacrum $\underline{\text { Elvan } \ddot{O}^{1}}$, Bobuş Örs A², Uzmansel D²

${ }^{1}$ School of Health, Mersin University, Mersin, Turkey, ${ }^{2}$ Department of Anatomy, School of Medicine, Mersin University, Mersin, Turkey

Objective: The aim of this study is to reveal the macroscopic variations of certain anatomical structures in the dorsal surface of the sacral bone.

Methods: Twenty dry sacrums with undetermined gender, present in the collection of Anatomy Department, School of Medicine, Mersin University were evaluated. Sacral canal, posterior sacral foramina, sacral hiatus, sacral cornu and superior articular process located in the dorsal surface of the sacrum were examined observationally.

Results: There was sacralisation in 3 of sacrum and 1 spina bifida occulta. The level of maximum curvature of sacrum was detected in S3 level as 60\% and S4 level as 25\%; no curvature in $15 \%$. Upper apertures of sacral canals were in "U" $(26 \%)$ and "V" (74\%) shapes. Facies of processus articularis posterior were detected as bilateral flat (35\%) and concave (55\%); and unilateral concave $(10 \%)$. Some of the sacrums were found to be united with the lumbar vertebra (10\%) and with coccyx (35\%). Accordingly, it was determined that the numbers of posterior sacral foramina varied. Shape differences were also observed in these foramina (oval, round, triangular, irregular). In general, the foramina were observed at the $\mathrm{S} 1$ level as oval in the vertical direction and those at the S4-5 level as oval in the horizontal direction. The shape of sacral hiatus was observed as inverted "U" and "Dumbbell" (28\%), inverted "V" and "M" (17\%) and irregular (12\%). Sacral cornu was detected in $78 \%$ sacrums. In $22 \%$ of sacrums, sacral cornu and coccygeal cornu were united. Apex of sacrum was detected most often at S4 (42\%) at least between S2 - S3 and at S5 (5\%) levels. Base of the sacrum was located at S4 (32\%) and S5 (68\%) levels.

Conclusion: In this study as we presented preliminary findings, there were many variations on the dorsal surface of the sacrum. Knowing the variations of these anatomical structures is suggested to help to prevent complications such as soft tissue injuries or local anaesthetic toxicity or to determine the local anaesthetic procedure options.

Keywords: sacrum; morphology; variation; sacral hiatus

\section{$0-90$}

\section{Morphometric evaluation of nutrient foramen in hip bone's gluteal surface}

Yücedağ H, Paslı B, Abbasov Taşkındere T, Güneş Erçakmak B Department of Anatomy, Faculty of Medicine, Hacettepe University, Ankara, Turkey

Objective: Vascularization of the bones are provided by nutrient arteries which are entering from nutrient foramina. This arterial supply begins from the early stages of ossification. Knowledge of the location and number of nutrient foramina is important to maintain the nutrition of the bone during certain surgeries such as bone graft, tumor resection or during the treatment of trauma and fractures. It is known that due to the hip traumas, injuries and surgeries through posterior approach, the superior gluteal artery can be damaged. In such cases, the nutrient arteries which are responsible for the supply of the hip bone can also be injured. The aim of this study is to evaluate the numbers and localizations of nutrient foramina at the gluteal surface of the ilium and distances between these foramina and certain anatomical landmarks.

Methods: This study was performed on 52 dry coxae of unknown age and gender that are belonging to the collection of dry bones throughout many years that have been stated in Hacettepe University, Faculty of Medicine, Department of Anatomy. The numbers and localizations of nutrient foramina that are greater than $1.5 \mathrm{~mm}$ in diameter were evaluated with naked eye and distances to the certain anatomical points (acetabulum, the most superior point of of the sacropelvic surface, midpoint of the line between posterior superior iliac spine and posterior inferior iliac spine, anterior superior iliac spine) was recorded. Gluteal surface of the ilium was divided into 4 regions according to the posterior- anterior- inferior gluteal lines and the localization of the nutrient foramina according to these regions were determined. All measurements were performed with a digital caliper with the accuracy $0.01 \mathrm{~mm}$. Statistical analyses were performed by using 'Statistical Package for Social Sciences for Windows v23.0' software.

Results: The number of foramina were 1 in $7.69 \%, 2-3$ in $71.15 \%$, and more than $4(4,5,6)$ in $21.15 \%$ of the coxae. One hundred and fifty foramina with a diameter greater than 1.5 $\mathrm{mm}$ were found. The most common location was the region under the inferior gluteal line $(44.30 \%)$. According to the regions, the mean distances of foramina to the reference points were determined.

Conclusion: In surgical procedures related to fractures of acetabulum and coxae, the superior gluteal artery must be preserved. In addition, it is important to protect the nutrient branches of bones. Determining the anatomical location of nutrient foramina and risky areas will reduce intraoperative and postoperative complications.

Keywords: hip bone, gluteal region, nutrient foramen, nutrient artery

\section{0-91}

Intercondylar fossa shape analysis: the differences between genders and their relationship with distal femoral morphometric parameters

İpek ED, Kızılay ÖC, Başaloğlu H

Department of Anatomy, Faculty of Medicine, Aydın Adnan Menderes University, Aydın, Turkey 
Objective: Intercondylar fossa (FI) anatomy plays an important role in anterior cruciate ligament (LCA) pathologies, as well as limiting its intra articular reconstruction applications. It is not recommended to perform double-beam reconstruction in LCA patients with FI width less than $12 \mathrm{~mm}$. Individualizing reconstructive surgery according to the patient's anatomy with guidance of morphometric research, increases the success of LCA reconstructions which have postoperative failure rates of up to $14 \%$. In our study, besides classifying the FI shape, we aimed to investigate its morphometric properties and possible correlations with distal femur.

Methods: For this study, adult femurs of 80 females (40 right, 40 left) and 80 males (40 right, 40 left), without any structural or acquired deformities and pathological changes were selected from the archive of our department. Distal surface of femurs placed anteroposteriorly on a flat surface, was automatically photographed from a fixed distance. Intercondylar fossa shape, width (w), height (h), area (LA, MA), angle (IFA) were measured using the Image $1.7 .0 \_45$ program. In addition, inclination angle and width (LM, MM) of lateral (LIA) and medial condyles (MIA) were measured. The variables which were normally distributed in groups were evaluated with One Way ANOVA, other variables were evaluated with Kruskal Wallis and their relationships with each other were evaluated using Pearson correlation test using SPSS.v21.

Results: Among samples, the most common form of FI was 'A' (43.8\%) shape and the least common form was ' $W$ ' (4.4\%) shape. While there was a significant difference between IFA, $h$ and $w$ parameters and FI shape, IFA was not significantly different between genders. There was a poor correlation between LIA and MIA, but no difference was found between genders and FI types in both measurements. Interestingly, LM and MM did not differ between FI types, a moderate correlation was found between w and $h$ values $(r=0.550, r=0.458)$. Lateral FI area was found to be different between genders, the medial FI area was also found to be different on the right and left sides in men $(\mathrm{p}<0.05)$.

Conclusion: Our study shows that the anatomy of FI is highly variational not only between genders but also within the same gender. A weak positive correlation was found between condylar inclination and FI shape and size. However, the lateral and medial condylar width was moderately positively correlated with the height and width of the FI. We think that the results of our research will contribute to studies on FI anatomy.

Keywords: intercondylar fossa, distal femur; morphometry; shape analysis

\section{0-92}

\section{Morphometric evaluation and clinical implications of} nutrient foramen on dry tibia

Ülkir $\mathrm{M}^{1}$, Karaçoban $\mathrm{L}^{2}$, Demiryürek D1

'Department of Anatomy, Faculty of Medicine, Hacettepe University, Ankara, Turkey, ${ }^{2}$ Department of Sports Medicine, Faculty of Medicine, Hacettepe University, Ankara, Turkey
Objective: Tibia is an important bone in the leg. Since its weight-bearing role, humans can keep the bipedal posture and walk kinematically. It is also one of the most robust bones in the body. This important bone needs blood supply for growth and metabolism, and the nutrient artery is responsible for this duty. The nutrient artery is the main blood supply of a long bone that enters the bone through the nutrient foramen. This foramen is located away from the growing end in the majority of cases; therefore, the derivation of the axiom that foramen "seeks the elbow and flees from the knee". The direction of the nutrient canal is crucial to identify the growing end of a bone.

Methods: This study was performed on 63 dry tibia bones (28 right, 35 left) at the Faculty of Medicine, Department of Anatomy, Hacettepe University. Eleven parameters related to nutrient foramen localization, size and morphometry were evaluated. Statistical analyses were performed using SPSS software version 22 (IBM, Armonk, NY). The visual (histograms, probability plots) and analytical methods (KolmogorovSimirnov/Shapiro-Wilk's test) were used to determine whether or not the parameters are normally distributed. Descriptive statistics were presented for normally distributed variables as mean \pm standard deviation; non-normal distributions using median and minimum-maximum values. The Mann-Whitney $\mathrm{U}$ test was used to compare the 11 parameters.

Results: One nutrient foramen was detected in $92.06 \%$ of all tibiae, 25 in the right tibia and 33 in the left tibia. Two nutrient foramina were detected in $7.96 \%$ of all tibiae, 3 in the right tibia and 2 in the left tibia. The length of the right tibia was $337.68 \pm 23.73 \mathrm{~mm}$, and the length of the left tibia was $345.24 \pm 24.43 \mathrm{~mm}$.

Conclusion: Tibial morphological properties of nutrient foramen may also give knowledge on forensic medicine, medicolegal issues, and anthropological studies. The information about the location and morphological properties of the nutrient foramen in tibia is therefore essential in joint replacement surgery, fracture repair, bone grafts, and vascularized bone microsurgery as well as forensic science cases

Keywords: tibia, nutrient foramen, nutrient artery, anatomy, morphometry

\section{0-93}

Reappraisal of the proximal tibia anatomy

Akdemir Aktaş H, Ülkir M, Günenç Beşer C, Demiryürek D

Department of Anatomy, Faculty of Medicine, Hacettepe University, Ankara, Turkey

Objective: Total knee arthroplasty has been popular in recent years. The morphometry of proximal tibia is important for surgeons to perform successful total knee arthroplasty. The aim of this study was to reappraise the proximal tibia morphometry.

Methods: In this study, 57 human dry tibia were evaluated. The largest anteroposterior and mediolateral dimensions of 
tibial condyles, maximum width of superior articular surface, and length of the tibia were measured. Also, morphometric measurements of intercondylar areas were done. All measurements were performed using a digital caliper.

Results: The mean values of the largest anteroposterior and mediolateral dimensions of medial tibial condyle were found $39.76 \pm 4.10 \mathrm{~mm}$ and $23.27 \pm 2.63 \mathrm{~mm}$, respectively. The mean values of the largest anteroposterior and mediolateral dimensions of lateral tibial condyle were measured $34.72 \pm 3.51 \mathrm{~mm}$ and $21.83 \pm 2.52 \mathrm{~mm}$, respectively. The maximum width of the superior articular surface was $65.14 \pm 4.70 \mathrm{~mm}$ and the length of the tibia was $34.12 \pm 2.32 \mathrm{~cm}$. The mean value of the anteroposterior dimension of the intercondylar area was found $41.62 \pm$ $4.27 \mathrm{~mm}$. The ratio of maximum width of superior articular surface to anteroposterior dimension of intercondylar area was found 1.57. The mediolateral dimensions of intercondylar area at anterior, middle, and posterior parts were measured $24.86 \pm$ $4.04,21.27 \pm 1.82$ and $21.36 \pm 4.04 \mathrm{~mm}$, respectively. The anteroposterior and mediolateral dimensions were significantly higher $(\mathrm{p}<0.05)$ for the medial condyle. The correlations between the tibial length and the dimensions of tibial condyles, maximum width of superior articular surface, anteroposterior dimension of intercondylar area were evaluated. Positive and statistically significant correlations were found between the tibial length and the other parameters $(\mathrm{p}<0.05, \mathrm{r}>0.40)$.

Conclusion: The morphometric data of tibial plateau are important for surgeons during meniscal allograft transplantation and total knee arthroplasty. A better understanding of proximal tibial anatomy is essential for designing appropriate tibial implants. Appropriate tibial implants will ensure the longevity of knee prosthesis and provide rapid patient mobili- ty. With many new advancements in arthroplasty technology, patient-specific prosthetic components may eradicate implant mismatch in the near future.

Keywords: total knee arthroplasty; proximal tibia; morphometry; intercondylar area

\section{0-94}

Traditional and complementary medicine and acupuncture with an anatomist's scientific view

\section{Kalaycioğlu A}

Department of Anatomy, Medical School, Biruni University, Istanbul, Turkey

Traditional and Complementary Medicine practices officially started in our country in 1992 with the Acupuncture regulation. A new regulation was prepared again in 2017 and its scope was expanded. This regulation has been published in the Official Gazette dated 27.10.2017 and numbered 29158. This regulation includes: acupuncture, apitherapy, phytotherapy, hypnosis, homeopathy, prolotherapy, osteopathy, ozone application, reflexology, music therapy. One of the oldest of these applications is acupuncture. To understand acupuncture, it is necessary to master human anatomy and especially neuroanatomy. I wonder what kind of relevance the facial structure, peripheral nervous system, central nervous system, and the connections of the organs may have in the acupuncture mechanism. What is the function of formatio reticularis in this mechanism?

Keywords: anatomy; acupuncture; traditional medicine; complementary medicine 


\section{Poster Presentations}

\section{(P-01 - P-38)}

\section{P-01}

Differences in anatomy terminology between anatomy textbooks and clinical resources: a preliminary study

Sağlam L, Coşkun Ö, Yiğit M, Gürses İA

Department of Anatomy, Istanbul Faculty of Medicine, Istanbul University, Istanbul, Turkey

Objective: In line with the clinical need, the anatomical structures defined in clinical books can be more detailed and different compared to the anatomical structures defined in Terminologia Anatomica (TA). In this study, comparison of the anatomical knowledge and terminology between clinical and anatomical resources was aimed with a limited example to evaluate the current situation.

Methods: To limit the study, a major joint and its ligaments were selected from the lower extremity. Accordingly, a list of ligaments under the title Ankle Joint of the TA published in 2011 was prepared. Seven basic anatomy resources (2 textbooks, 3 atlases and 2 cadaver atlases) and 3 orthopedic textbooks on arthroscopy and endoscopy of the ankle published after 2011 was compared.

Results: Ten terms describing the ankle ligaments in TA (including the title) were observed. The coverage ratio of selected sources on the 10 identified terms were 10/10 (100\%) for Clinically Oriented Anatomy, 10/10 (100\%) for Gray's Anatomy, 9/10 (90\%) for Sobotta Atlas of Human Anatomy, 9/10 (90\%) for Thieme Atlas of Anatomy, 8/10 (80\%) for Anatomy a Photographic Atlas, and 6/10 (60\%) for Human Anatomy Color Atlas and Textbook. The coverage of clinical sources were 8/10 (80\%) for Ankle Arthroscopy Techniques Developed by the Amsterdam Foot and Ankle School, 10/10 (100\%) for Ankle Joint Arthroscopy A Step-by-Step Guide, and 8/10 (80\%) for Arthroscopy and Endoscopy of the Foot and Ankle Principle and Practice. Gray's Anatomy, Ankle Joint Arthroscopy A Step-by-Step Guide, Ankle Arthroscopy Techniques Developed by the Amsterdam Foot and Ankle School, and Arthroscopy and Endoscopy of the Foot and Ankle Principle and Practice named additional 3, 3, 2, and 1 structures, respectively. Of these additional 10 terms, 7 were subcomponents of the medial collateral ligament and 3 were the subcomponents of the lateral collateral ligament.

Conclusion: It was expected to see a more detailed terminology in orthopedic resources and Gray's Anatomy, since they provide literature-based information and focus on postgraduate education. However, if it is considered that TA is a valid guide for all disciplines, it can be suggested that the new nomenclature in these textbooks should be evaluated and possibly included in the scope of the TA. Additionally, studies to detect the prevalence of similar inconsistencies in terms regarding different topographic regions or systems may be planned.

Keywords: terminologia anatomica, terminology, nomenclature, atlas, textbook

\section{P-02}

Investigation of the effect of anatomy education on body awareness

Dinç $\mathrm{M}^{1}$, Vurgun, $\mathrm{H}^{2}$, Maral $\mathrm{F}^{3}$, Arifoğlu $\mathrm{Y}^{3}$

'Physiotherapy Program, Vocational School, Doğuş University, Istanbul, Turkey; 'Department of Anatomy, Faculty of Medicine, Biruni University, Istanbul, Turkey; ${ }^{3}$ Department of Anatomy, Faculty of Medicine, Bezmialem Vakıf University, Istanbul, Turkey

Objective: This study was conducted to examine the body awareness levels of university students who took and did not take anatomy lessons.

Methods: A total of 296 healthy and voluntary university students between the ages of 18-35 with no known diseases; 148 of which, consisting of 94 females and 54 males, took anatomy lessons and 148 of which, consisting of 96 females and 52 males, did not take any anatomy lessons were included in the study. Sociodemographic information of the participants were recorded with the prepared form. The body awareness level of each participant was evaluated using the Body Awareness Questionnaire (BAQ), which was developed in 1989 by Shields, Mallory \& Simon, and whose validity and reliability were made in Turkish. The questionnaire consists of four sub (attention to changes in body processes and reactions, sleep-wake cycle, prediction at the onset of the disease, estimation of body responses) and 18 statements in total, and between one and seven evaluations for each statement ( 1 = I strongly disagree, 7 = I strongly agree) were done. The rating is based on the total score obtained and a maximum of 126 and a minimum of 18 points can be obtained. It is concluded that the higher the total score obtained from the questionnaire, the better the body awareness. Statistical analysis of the data obtained was made with the SPSS 27.0 package program. Kolmogorov-Smirnov Analysis, which is a normality test, was used for the compliance of individuals' body awareness levels to parametric or non-parametric tests, and the Mann Whitney U Test was used to make comparisons between groups.

Results: When the data obtained from the individuals who participated in the study were examined, the body awareness score of the group receiving anatomy training was $102.52 \pm 12.40$; Body awareness score of the group who did not receive anatomy training was found to be $85.87 \pm 15.63$. When these two groups were 
compared, it was found that the body awareness scores of the group that received anatomy training were statistically significantly superior to the group who did not receive anatomy training $(\mathrm{p}<0.05)$.

Conclusion: According to the data obtained in the study, it was seen that anatomy education was effective on body awareness. The concept of body awareness includes the perception of normal or abnormal sensitivity of body composition. We believe that it would be appropriate to provide basic anatomy education to all university students in order to gain this awareness.

Keywords: anatomy education, body awareness, body perception, healthy adult

\section{P-03}

Anatomic structure of ulnar nerve at wrist level and location of its branches

Vural A ${ }^{1}$, Kaptan $\mathrm{AY}^{2}$, Kanatlı $\mathrm{U}^{3}$, $\underline{\text { Ince }}^{\mathrm{M}} \mathrm{S}^{4}$, Aytaç $\mathrm{G}^{5}$, Gözil R 'Dr. Sami Ulus Maternity, Child Health and Diseases Training and Research Hospital; ' ${ }^{2}$ Başakşehir Pine and Sakura City Hospital, ${ }^{3}$ Department of Orthopedics and Traumatology, Faculty of Medicine, Gazi University; ${ }^{4}$ Department of Physiotherapy and Rehabilitation, Faculty of Health Sciences, Yüksek ihtisas University; ${ }^{5}$ Department of Anatomy, Faculty of Medicine, Yüksek ihtisas University

Objective: The ulnar nerve gives its dorsal and palmar cutaneous branches before it reaches the wrist, and then enters the Guyon's canal along with the ulnar artery. Guyon's canal runs from the proximal edge of the volar carpal ligament to the fibrous arch of the hypothenar muscles. Volar carpal ligament and palmaris brevis are roof of the Guyon canal, flexor digitorum profundus tendons, transverse carpal, pisohamate, pisometacarpal ligaments and opponens digiti minimi are base, flexor carpi ulnaris, pisiform bone and abductor digiti minimi are medial wall, and flexor tendons, transverse carpal ligament and hamate bone are lateral wall. The ulnar nerve passing through the Guyon's canal (zone 1), first gives its deep branch (zone 2) and then its superficial sensory branch (zone 3). The deep branch is the motor branch and provides motor innervation of the hypothenar muscles, interosseous muscles, the $3 \mathrm{rd}$ and 4th lumbar muscles, the medial head of the adductor pollicis and flexor pollicis brevis. Dorsal and palmar cutaneous branches do not pass through the Guyon canal. The aim of our study is to determine the distance between the volar carpal ligament, and the deep branch of the ulnar nerve and between the volar carpal ligament and the bifurcation of the superficial branch of the ulnar nerve.

Methods: Our study was carried out on 9 fresh upper extremity cadavers in the Anatomy Laboratory of the Yüksek İhtisas University. First, the palmar branches of the ulnar nerve were made visible in cadavers whose hand palmar surfaces were dissected while preserving the volar carpal ligament. Afterwards, the distance of the bifurcations of the deep branch in zone 2 and the superficial branch in zone 3 to the volar carpal ligament was measured. Since there are microbrances after bifurcatio, measurements were made until bifurcatio. In addition, the length of the volar carpal ligament was measured. The measurements were carried out by the same anatomist, and each parameter was measured three times, and the average of three measurements was evaluated.

Results: As a result of our study, the mean distance between the volar carpal ligament and the deep branch of the ulnar nerve was $5.9 \pm 3 \mathrm{~mm}$, and the mean distance between the bifurcation of the superficial branch of the ulnar nerve was $14.3 \pm 4.4 \mathrm{~mm}$. The average length of the volar carpal ligament was determined as $13.6 \pm 2.3 \mathrm{~mm}$.

Conclusion: The second area where the ulnar nerve is most frequently trapped is the wrist. While ulnar nerve entrapment in the wrist is most common in the Guyon region, entrapment in the distal region can give different clinical findings. In clinical evaluation, while sensory and motor findings are seen together in zone 1 entrapments, only motor findings in zone 2 and only sensory findings in zone 3 are observed. Our study results provide information about the anatomical structure of the distribution of the ulnar nerve in the Guyon's canal and its distal. We think that these results may guide the treatment of different region entrapments on the wrist.

Keywords: ulnar nerve, Guyon's canal, morphometry, anatomy

\section{P-04}

Investigation of mobile technology usage attitudes of 2nd year students of NEU Faculty of Medicine

Yılmaz İ, Öztürk Y, Lafçı Fahrioglu S, İlgi S

Department of Anatomy, Faculty of Medicine, Near East University, Nicosia, TRNC

Objective: Covid-19 first appeared in December 2019 and turned into a pandemic as of March 2020. Although the impact of the pandemic was primarily seen in social life with the social isolation measure taken, in the following period, it was also effective in the fields of economy, work and education and the usual life order changed in many areas. According to UNESCO data, the education of $90 \%$ of students (from pre-school to higher education) all over the world has been affected by pandemic conditions. Like many educational institutions, Near East University has decided to continue education with the online education system on March 16, 2020 in order to compensate for educational opportunities. In order to reveal the potential of the students taking anatomy lessons in this period to adapt to this new system, this survey study was planned to examine the attitudes of the Near East University Faculty of Medicine 2nd grade students on the use of mobile learning technologies.

Methods: One-hundred-ten students who answered the questionnaire used by the researchers among the second year students of NEU Faculty of Medicine were included in the study. The age-gender characteristics of the students and the experi- 
ence, competence and expertise levels of mobile technologies (smart phone, tablet, etc.) were investigated, followed by the effect of mobile technology use on effectiveness, performance, efficiency and productivity on education with a 5-Likert scale.

Results: The average age of the participants in the study was $23.17 \pm 2.11,55 \%$ of the students were male and $45 \%$ of the students were female. In terms of the level of experience in using mobile technologies, $67 \%$ of the students rated their experience level, $68 \%$ their proficiency level, and $42 \%$ their expertise level as "good-very good". Seventy percent $(70 \%)$ of the students stated that using mobile technologies for lessons increased their performance, $77 \%$ of the students stated increased efficiency in accessing the course materials, $51 \%$ of the students stated increased their effectiveness in the lessons, and $59 \%$ of the students stated increased the quality of the education they received. In addition, $86 \%$ of the students stated that their participation in the lessons using mobile technologies can be realized when the necessary devices and resources are provided, $82 \%$ of them can get technical support when they need it is very effective in their use of mobile technology, $84 \%$ of them can use it to educate if the mobile technology they use is not complicated. Ten percent (10\%) of the students do not trust themselves to carry out learning activities with mobile technologies, $13 \%$ of them do not see themselves as having the competence to learn with mobile technologies successfully, and $24 \%$ of them do not actually like to learn using mobile technologies, We learned that $16 \%$ of them do not plan to use mobile technologies and $83 \%$ of them they can use mobile technologies in their future education life.

Conclusion: Although the online education which started at the NEU Faculty of Medicine in March 2020, due to the pandemic, can be considered as a compulsory situation due to the tendency of the young generation to mobile devices, it is still a member of this young generation, but to accept online education due to necessity. The existence of the forced young people should not be forgotten. In addition, it was decided to continue the education of our faculty by using face-to-face and online systems together during the pandemic period.

Keywords: pandemic, faculty of medicine, on-line education, mobile technology

\section{P-05}

A right coronary artery originating from left anterior descending artery (LAD): a case report

Lafç Fahrioğlu S ${ }^{1}$, Muhtaroğlu $\mathrm{M}^{1}$, Beton $\mathrm{O}^{2}$, İlgi $\mathrm{S}^{1}$

'Department of Anatomy, Faculty of Medicine, Near East University, Nicosia, TRNC; ${ }^{2}$ Dr. Burhan Nalbantoğlu State Hospital Cardiology Department, Nicosia, TRNC

Objective: Variations of the coronary arteries are rare variations that affect around $0.64-1.3 \%$ of patients and are usually diagnosed by incidentally during a coronary angiography or autopsy. Coronary anomalies are classified according to their origin, course and distribution. Anomalous origins of the coro- nary arteries are classified considering the number of the ostia and the ostial positioning. The anomalous origin of the right coronary artery (RCA) as a branch from the left anterior descending artery (LAD) is a rare variation of the single coronary artery anomaly with the incidence of $0.019 \%$ to $0.49 \%$ on coronary angiography.

Case: A 65-year-old male patient was admitted to the cardiology outpatient clinic with chest pain. Neither did he have a history of coronary artery disease or smoking, nor were any anomalies found in his echocardiography or electrocardiography. A coronary angiography was performed on the patient who had effort angina, a coronary artery disease risk factor. In the left system injection, it was observed that the left anterior descending (LAD) and the circumflex arteries (Cx) were originating from the left coronary sinus with separate ostia. The right coronary artery was a branch of the LAD. A coronary ostium could not be detected in the right injection. No constrictions in the coronary arteries were diagnosed. The informed consent was obtained from the patient.

Conclusion: Due to the fact that anomalies of the coronary artery origins are uncommon, its clinical and anatomical significance has not yet been specified. In addition, even though the role in which this kind of coronary artery anomaly would have in the development of atherosclerosis has not been clearly determined; if in these cases coronary artery surgery will be performed, which coronary artery to greft must be thoroughly considered. In case the anomalous origins of the coronary arteries are not diagnosed in a preoperative period, they might cause significant complications during the intraoperative or postoperative period

Keywords: coronary artery, variation, coronary angiography, chest pain

\section{P-06}

An evaluation of articles on plastic, reconstructive and aesthetic surgery published in anatomy journals indexed in $\mathrm{SCl}$ and $\mathrm{SCl}-\mathrm{E}$

Öner Ç, Karip B, Balcığlu HA

Department of Anatomy, Faculty of Medicine, University of Health Sciences, Istanbul, Turkey

Objective: The aim of this study is to examine the articles in international scientific journals in the field of Anatomy in the last 10 years, plastic, reconstructive and aesthetic surgery; number, ratio, distribution by country, subject and method of the studies conducted.

Methods: Journals indexed within the scope of "science citation index" (SCI) and "science citation index expanded" (SCI-E), which are in the "anatomy and morphology" category from the Web of Science (WoS) online database, were included in the study. These journals were searched using the keywords "plastic surgery", "reconstructive surgery" and "hand surgery" separately and all together. As a result of the search, the weight of the articles produced with anatomical studies was evaluated in the rele- 
vant journal. The articles in question were evaluated according to the publishing country, study topic, study method and the contribution of the anatomy departments in the relevant articles.

Results: Among the journals published in the field of anatomy since 2010, 23 journals covered by "science citation index" (SCI) and science citation index expanded (SCI-E) were found. It was determined that 9 of them were indexed under SCI-E and 14 of them were indexed under both SCI and SCI-I. As a result of the search with keywords in these journals, 4981 articles related to plastic, reconstructive and aesthetic surgery were found. It was evaluated that there were no studies based on anatomy in 8 out of the 23 journals detected. The rates of the total number of articles obtained by scanning the keywords separately and scanning them together were determined. It has been observed that these rates have increased especially in the last 5 years compared to the previous 5 years.

Conclusion: The significance achieved as a result of these studies emphasizes the importance of anatomical sciences and especially cadaver education. Anatomical studies in all clinical branches are still up-to-date on scientific publication platforms. Keywords: anatomical sciences, plastic and aesthetic surgery, Web of Science

\section{P-07}

Vascular anatomy of the liver: radiological pictorial assay

Kesimal U

Department of Radiology, Faculty of Medicine, Recep Tayyip Erdogan University Training and Research Hospital, Rize, Turkey

Objective: Our aim in our study is to demonstrate the hepatic vascular (arterial, venous, portal venous) anatomy in our large series of patients using radiological methods. Hepatic vascular (hepatic arterial and venous, portal venous) variations play a critical role in evaluating the liver before surgical interventions, transplantation and invasive procedures. While the lack of knowledge about these variations can be dangerous during these procedures, being aware of them can help reduce complications.

Methods: In addition to the developments in computer and imaging techniques, three-dimensional (3D) imaging has been made possible with the developments in multi-detector computed tomography (MDCT) systems. 3D imaging techniques such as maximum intensity projection (MIP), multiplanar reconstruction (MPR), and volume rendering (VR) provide detailed imaging of vascular structures with MDCT. In our study, we demonstrated hepatic vascular anatomy with our MDCT images.

Conclusion: We present 8 cases with hepatic arterial anatomy according to Michel classification, 3 cases with portal venous anatomy and 3 cases with hepatic venous anatomy and our cases including MIP, MPR and 3D MDCT images and some surgical images of 14 cases in total.

Results: Hepatic vascular variations are usually detected at routine examinations as a result of advances in non-invasive, crosssectional imaging techniques.
Keywords: vascular anatomy of the liver, radiological pictorial assay

\section{P-08}

Determination of the frequency of "coeliac artery compression syndrome" by computed tomography angiography (Preliminary study)

Taşpınar $C^{1}$, Uysal $\mathrm{II}^{1}$, Seher $\mathrm{N}^{2}$, Koplay $\mathrm{M}^{2}$

${ }^{\prime}$ Department of Anatomy, Meram Faculty of Medicine, Necmettin Erbakan University, Konya, Turkey; ${ }^{2}$ Department of Radiology, Faculty of Medicine, Selçuk University, Konya, Turkey

Objective: A cause of gastrointestinal ischemia is compression of the proximal part of the truncus coeliacus by ligamentum arcuatum medianum. This circulatory disorder, called coeliac artery compression syndrome or median arcuate ligament syndrome (MALS), may be asymptomatic or cause epigastric pain, weight loss, and abdominal discomfort. In this study, it was aimed to determine the frequency and compression rates of MALS by computed tomography (CT) angiography.

Methods: With the permission of the local ethics committee, abdominal CT angiography (2018-2020) in the archive of the Radiology Department of Selçuk University Faculty of Medicine was examined. The relationship between the truncus coeliacus and the ligamentum arcuatum medianum was evaluated in sagittal section images of 238 patients (103 females, 135 males, 32-73 years old) included in this retrospective study.

Results: In the study, in 15 of 238 cases (6 female, 9 male), it was observed that the ligamentum arcuatum medianum caused compression of the truncus coeliacus at varying rates. Compression was detected in $50 \%$ in 6 cases ( 2 female, 4 male), $60 \%$ in 2 patients ( 1 female, 1 male), $70 \%$ in 4 patients (2 female, 2 male) and $80 \%$ in 3 patients ( 1 female, 2 male).

Conclusion: In line with the preliminary data obtained from the ongoing study, the presence, frequency and compression rates of MALS in CT angiography images should be considered in patients whose etiology of abdominal pain is investigated.

Keywords: truncus coeliacus, ligamentum arcuatum medianum, MALS

\section{P-09}

Posterolateral corner anatomy of the knee joint and its clinical significance: a literature review

Canlı A, Vatansever A

Department of Anatomy, Faculty of Medicine, Balıkesir University, Balıkesir, Turkey

Objective: Posterolateral corner of knee joint (PLC), the least understood area of knee joint, has been paid more attention in recent studies. Therefore, it is also referred to as a dark field. In recent years, the role of PLC structures in knee stability has attracted more attention and studies have increased to this extent. 
It has been demonstrated that PLC injury, which was ignored in previous years, had caused a wide variety of unwanted postoperative complications. The purpose of this literature review is to present up-to-date information about the anatomy and biomechanics of this region, which has been done on PLC in recent years.

Methods: Recent studies were compiled using the keywords "posterolateral corner", "collateral ligament", "popliteal tendon", "fabellafibular ligament", "knee", "biomechanical study of posterolateral corner".

Results: Better understanding of anatomy, biomechanical properties and variations of PLC related structures, and increased awareness on these structures increase the success of diagnosis and treatment of PLC pathologies. Anterior and posterior cruciate ligaments' injuries may be accompanied by damage to PLC structures.

Conclusion: Isolated PLC damage was rarely observed. Injuries to these structures were seen more often (43\% - 80\%) associated with damage to other ligaments of the knee joint, including damage to the posterior or anterior cruciate ligaments. The fact that some anatomical structures that are not included in the classical anatomy textbooks, thus, using different terminology could cause a complexity in the literature. For example, popliteofibular ligament (PFL) referred as external ligament, popliteofibular fascicle, fibular origin of popliteus muscle, popliteofibular fibers for fibular head of popliteus muscle in current literature. Seebacher et al. defined the anatomical structures of PLC as three layers in their cadaveric study in 1982. More recently, three main structures have been identified as the primary stabilizers of PLC. these structures have been defined as lateral collateral ligament, tendon popliteus muscle and popliteofibular ligament in biomechanical studies. Recently defined meniscofibular ligament, MFL; is a capsular ligament originating from lateral meniscus in front of the popliteus tendon, has not a common consensus whether it should be included in PLC structures, yet. In biomechanical studies, it was observed that PLC structures of the knee joint prevent varus of knee, external rotation and posterior translation of the tibia.

Keywords: posterolateral corner, collateral ligaments, popliteal tendon, fabellafibular ligament, knee, posterolateral corner biomechanics

\section{P-10}

Anatomy and function of sacrotuberous ligament: systematic review

$\underline{\text { Bedre }}^{1}$, Ipor GT ${ }^{2}$, Dönmez BO ${ }^{2}$

'Department of Anatomy, Faculty of Medicine, Bakırçay University, Izmir, Turkey; '2Department of Anatomy, Faculty of Medicine, Pamukkale University, Izmir, Turkey

Objective: Sacrotuberous ligament is a strong ligament that provides stability to the sacroiliac region, by offsetting its rotation, therefore limiting the movement of the lower portion of sacrum.
İmportant structures which are close to the sacrotuberous ligament are the pudendal nerve, internal pudendal artery and coccygeal branch of inferior gluteal artery. Pudendal nerve may be compressed between the sacrospinal and the sacrotuberous ligaments. An ossified sacrotuberous ligament affects the general stability of the sacroiliac joint and this appears as an significant etiological factor in neurovascular compression syndromes. This study aims to investigate current understanding of the sacrotuberous ligament, puts into light on insufficient parts of its anatomy and function and to determine the study fields that require detailed research.

Methods: In this systematic review, 107 studies published between 2000-2020 were analyzed. It has been searched using the keyword "sacrotuberous ligament" in the US National Institutes of Health's National Library of Medicine" data base. Although the number of articles examined in the study was 107, 20 studies on the anatomy and function of the sacrotuberous ligament were included.

Results: Sacrotuberous ligament length, height and cross-sectional area showed sex-based differences at statistically significant level according to results of studies about sacrotuberous ligament provided by $3 \mathrm{D}$ imaging method. When the morphology based studies were analyzed, length $(6.4-9.4 \mathrm{~cm})$, depth $(0.3-0.4 \mathrm{~cm})$ and width $(1.8-3.5 \mathrm{~cm})$ of the STL showed some differences. Datas about the cause of different results obtained by 3D imaging method are limited considerably. Pelvic pain due to chronic pudendal nerve compression, when treated surgically, is approached with a transgluteal division of the sacrotuberous ligament. Surgically divided STL is significantly thicker after healing. Sacrotuberous ligament is pierced by inferior and superior gluteal artery but information on its innervation pattern is restrictive. Studies about this are insufficient. Sacrotuberous ligament, sacrospinous ligaments, iliolumbar ligaments and dorsal and ventral sacroiliac ligament play a role in locking or in allowing motion of the sacroiliac joint. Studies on analysis of sacrotuberous ligament on clinical conditions affecting sacroiliac joint have been found to be insufficient.

Conclusion: The anatomical and functional knowledge of STL may be useful for clinicians, radiologists and surgeons. It is required to investigate some morphological aspects of the STL. Especially studies on clinical conditions affecting sacroiliac joint should be performed on morphometric analysis of sacrotuberous ligament, its connections with surrounding structures, innervation pattern and function.

Keywords: sacrotuberous ligament; pudendal nerve; ossification; pelvic stability.

\section{P-11}

Current overview of the clinical importance of the anorectal angle

Girgin T, Vatansever A, Özcan E

Department of Anatomy, Faculty of Medicine, Balıkesir University, Balıkesir, Turkey 
Objective: The anorectal angle arises from tonic contraction of puborectalis muscle and traction of puborectal sling to the anorectal junction. Anorectal angle is defined as the angle between the central axis of canalis analis and line tangent to the posterior wall of rectum. Changing of anorectal angle during straining and defecation phases is clinically important in individuals with dyssynergic defecation. Puborectal muscle, which forms the anorectal angle during resting phase, is very important in ensuring fecal continence. Liu, M et al. studied in 2019 emphasized the clinical importance of anorectal angle in chronic constipation and Kollmann, C et al. studied in 2020 in fecal incontinence. In addition, Yamamota, T et al. Studied in 2020 has been reported that the anorectal angle is a predictive parameter for minimally invasive rectal cancer surgery difficulty level. Aim of this review: To examine the effect of the anatomical relationship between musculus puborectalis and anorectal angle on current clinical approaches.

Methods: Using the terms 'musculus puborectalis', 'anorectal angle', 'clinical anatomy', 'surgical' as keywords a literature review has been done. As a result of the literature review, current articles were included in our review.

Results: Normal value of anorectal angle is 90-110 degrees in resting position, and this angle changes 15-20 degrees in squeezing and defecation phases. In individuals with idiopathic fecal incontinence, an average resting phase anorectal angle of 134 degrees is determinant of success of sacral nerve stimulation. Anorectal angle of 123 degrees and above is a risk factor for minimally invasive rectal cancer surgery difficulty.

Conclusion: The anorectal angle, which increases in direct proportion to age, body mass index, and parity in young adults, is very important for both clinical and surgical processes. We think that knowing the normal values and changes of the anorectal angle in the resting, squeezing and defecation phases in healthy individuals will guide clinicians in understanding defecation pathologies.

Keywords: musculus puborectalis, anorectal angle, clinical anatomy, surgical

\section{P-12}

The importance of epiglottic cartilage in endotracheal tube application: a literature review

Derinöz E, Vatansever A, Kuş İ

Department of Anatomy, Faculty of Medicine, Balıkesir University, Balıkesir, Turkey

Objective: Epiglottic cartilage is a larynx cartilage with elastic cartilage structure and leaf-like appearance. The morphometric properties such as length, width and height of this structure and its clinical significance have been demonstrated by scientific research. With this review, it is aimed to raise awareness about epiglottic cartilage in terms of physicians while performing endotracheal intubation.
Methods: In the study, a literature search was conducted on internet based websites such as google scholar, pubmed, springer, karger journals, researchgate. In the research, studies carried out between 2000-2020 were examined. Literature was searched using the keywords "epiglottic cartilage, endotracheal tube", "epiglottis, morphometry", "epiglottis, anatomy", "epiglottis, calcification", "epiglottic cartilage, sleep apnea" and "epiglottis". In this compilation study; Epiglottis anatomy and calcification, epiglottis shape in patients with sleep apnea, epiglottic folds and injuries in endotracheal tube applications are discussed.

Results: Ten articles were included in the study as a result of the literature review. In one case study, ascending epiglottis was considered as a benign variant and no abnormal condition was found in the patient. Three forms of epiglottis have been identified in patients with sleep apnea. It was observed that the degree of obstruction was higher in patients with flat epiglottis shape. In a morphometric study in which the age-related changes of adult human epiglottis were examined using digital quantitative analysis of cartilage calcification, it was observed that calcification occurred in epiglottic cartilage due to increasing age. In another study, it was determined that epiglottis calcification causes dysphagia. In addition, there is a population study in Japan in which the thickness of epiglottic cartilage is measured using computed tomography images. Using ultrasound as an evaluation method to predict difficult intubation during endotracheal tube applications actually reveals that epiglottic cartilage damage is a structure that needs attention.

Conclusion: As a result of the research, it is emphasized that the epiglottic cartilage may be damaged or folded during the advancement of the endotracheal tube, regardless of which method is applied during intubation. In addition, it has been determined that epiglottis calcification may cause difficult intubation. Therefore, it is important for physicians to pay attention to this structure during intubation.

Keywords: Epiglottic cartilage; calcification; endotracheal tube; sleep apnea; morphometry; anatomy.

\section{P-13}

Discovery of ata ligament and spinal meningovertebral ligaments: a literature review

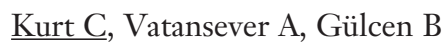

Department of Anatomy, Faculty of Medicine, Balıkesir University, Balıkesir, Turkey

Objective: ATA ligament, first discovered by Solaroglu, I et al 2011, is a keystone and could lead dural injuries during surgeries. Furthermore, the terms of 'meningovertebral ligaments' and 'myodural bridge' has been added to the literature by following studies, Benchao S et al 2012-2013 ; Scali, F et al 2015. ATA Ligament is mainly located between L5-S1 level, while the other ligaments could be seen in different vertebral levels. 
Aim of this review was to revisit current knowledge, such as anatomical locations and clinical importance, about recently discovered ATA ligament, meningovertebral ligaments and myodural bridge.

Methods: Using terms 'ATA ligament', meningovertebral ligaments', 'myodural bridge' as keywords a literature review has been done. Only articles after 2010 included a review.

Results: As a result of intraoperative and cadaveric studies; those anatomical structures were clearly monitored. Beside that cervical continuous of those ligaments was demonstrated.

Conclusion: With discovery of the ATA ligament, the concept of the meningovertebral ligament has been introduced to literature. It is very important in terms of preventing unexpected surgical complications, especially dural lacerations, which may have serious consequences. Moreover, examining the relationship of these structures, which also continues in the cervical region, with other possible organic diseases may also be crucial for physicians. Unfortunately, there is no study focused on those structures using radiology techniques, while most studies have used cadavers or intraoperative monitoring systems for demonstrating those structures. It has a significant importance to know about those ligaments' anatomical locations for surgeons to avoid iatrogenic injuries.

Keywords: dural injuries, ata ligament, meningovertebral ligaments, myodural bridge

\section{P-14}

\section{A silent disease: Leriche syndrome}

$\underline{\text { Digilli B }}^{1}$, Gökşan AS ${ }^{1}$, Çiçekcibaşı AE ${ }^{1}$, Koplay $\mathrm{M}^{2}$

'Department of Anatomy, Meram Faculty of Medicine, Necmettin Erbakan University, Konya, Turkey; ${ }^{2}$ Department of Radiology, Faculty of Medicine, Selçuk University, Konya, Turkey

Objective: Leriche syndrome (LS), mostly mentioned as aortoiliac occlusive disease (AITD), is a variant of peripheral artery disease $(\mathrm{PAD})$ characterized by atheromatous occlusion or stenosis of the infrarenal aorta and / or iliac arteries. According to the anatomical location of the occlusion, LS was defined as a syndrome by René Leriche with the triad of these symptoms; cramping leg pain which occurs with exercise and recovery with rest (claudication), sexual dysfunction and decreased-nonpalpable femoral pulses. The real prevalence and incidence of Leriche Syndrome are unknown since many patients are asymptomatic. On the other hand, multiple studies support that the prevalence of PAD increases in aging populations, $14 \%$ for patients older than 69 years of age and more prevalent in males. The aim of this study is to review the cases of elderly patients with Leriche syndrome using radiological imaging.

Methods: Reports of computed tomography angiography (CTA) images of Selçuk University Faculty of Medicine Department of Radiology and literature review.
Results: Leriche syndrome was observed on CT angiography images of 71 and 72-year old male patients. CTA showed left common iliac artery occlusion in 71-year old male patient and right common iliac artery occlusion in 72-year old male patient. Systemic and visceral collateralization pathways were seen on CT angiography in both patients.

Conclusion: Since Leriche syndrome is a progressive atherosclerotic disease, hypertension, hyperglycaemia, hyperlipidaemia, nicotine, age, gender and family history are the important risk factors for patients. Although atherosclerotic lesions obstruct the main vessels nourishing the lower extremities, the development of collateral arterial pathways provides perfusion to them. Therefore, LS is a silent disease and the onset of symptoms may be delayed in patients. As a result of decreased blood flow in LS, gangrene and amputation are increased in untreated patients, so medical and surgical treatment modalities should be started as quickly as possible. Aortoiliac bypass, aortobifemoral bypass (AFB), percutaneous transluminal angioplasty (PTA) and thromboendarterectomy (TEA) are the revascularization options for treatment of LS. It is vital to detect collateral pathways to the lower limbs during surgical interventions not to lead to any vascular injury resulting in significant morbidity, such as critical limb ischemia. CT angiography is very convenient to detect aortoiliac occlusions and their associated collateral pathways. Also, some vascular conditions such as arterial dissection, aneurysm, embolism, Takayasu and giant cell arteritis can mimic the symptoms of Leriche Syndrome and must be considered in the differential diagnosis.

Keywords: Leriche syndrome, CT angiography, claudication, common iliac artery

\section{P-15}

Effects of increasing electronic device use on head and neck anatomy

\section{Yazan H, Ayazoğlu M, Yılmaz V}

Department of Anatomy, Faculty of Medicine, Süleyman Demirel University, Isparta, Turkey

The use of electronic devices is quite common today and has become an indispensable part of our lives with the emergence of distance education and work due to the pandemic. The widespread use of these devices causes changes in the musculoskeletal system in the long run. The relationship between computer use and musculoskeletal disorders have been seen in the literature. Mechanical neck pain increased cervical lordosis, cervical spondylosis, and cervical disc hernia are the leading musculoskeletal problems seen in these individuals who took place in the studies. Mechanical influence anywhere in the skeletal system affects the entire system like a domino stone. The relationship between craniocervical posture changes and articulatio temporomandibularis has been discussed in the literature for years. Articulatio atlantooccipitalis and cervical ver- 
tebra movements are observed simultaneously with the activation of the masticatory muscles and the jaw joint. It has suggested that bad posture changes the position of the jaw by affecting the muscles, ligaments, and surrounding structures, thus causing the development of dysfunction by creating load on the articulatio temporomandibularis. An increase in head protrusion (forward head posture), and an increase in cranium extension (hyperlordosis) due to increased or decreased cervical angulation, and a decrease in the suboccipital distance are changes that are generally associated with those. Considering that Articulatio temporomandibularis irregularities are an important public health problem affecting approximately $5-12 \%$ of the society, the importance and investigation of the issue are imperative for future periods. Enthesis sites are sites where ligament, tendon, or joint capsule attach to a bone. Its function is to distribute the force over the bone surface. Its function is to distribute the force over the bone surface. In a radiographic study, calcification found in young adults around the pranotubertia occipitalis externa at the attachment point of the muscles and ligaments, which was not seen before the 2000s. This calcification, which has reached up to $2.6 \mathrm{~mm}$ as of 2020 , is formed to support excessively strained muscles. This calcification was positively associated with increased cervical angulation. The meaningful difference in young male individuals in this study suggests that the anatomical effects of electronic devices on different ages, societies, and genders are open to further research. Long hours spent in front of the computer and phone cause changes in the musculoskeletal system. Head and neck are some of the most affected areas. Considering that these effects will grow in the future, it is essential to know and investigate the anatomical effects both clinically and for anatomists.

Keywords: anatomical change, use of electronic devices, articulatio temporomandibularis, craniocervical posture

\section{P-16}

The relationship of anatomic localizations of esophageal hiatus and esophagogastric junction with body mass index: a preliminary study

$\underline{\text { Karaca Bozdağ } Z^{1}}$, Candır BN ${ }^{1}$, Bozdağ E², Kürkcüoğlu A3 Macunluoğlu $\mathrm{AC}^{4}$

'Department of Anatomy, Faculty of Medicine, Istanbul Yeni Yüzyıl University, Istanbul, Turkey; ${ }^{2}$ General Surgery Clinic, Research and Training Hospital of Istanbul Kanuni Sultan Süleyman, University of Health Science, Istanbul, Turkey; ${ }^{3}$ Department of Anatomy, Faculty of Medicine, Başkent University, Ankara, Turkey; ${ }^{4}$ Department of Biostatistics, Health Sciences Institute, Uludağ University, Bursa, Turkey

Objective: Endoscopy is accepted as the gold standard worldwide in the examination of the upper gastrointestinal system. Although there have been studies investigating the relation of body mass index (BMI) with various diseases, studies revealing its relation with anatomical structures are quite limited. In our study, we aimed to reveal the relationship between anatomic locations and $\mathrm{BMI}$ in patients planned for upper gastrointestinal endoscopy.

Methods: It was planned to investigate the anatomical localizations of the esophageal hiatus and esophagogastric junction depending on the BMI of 191 patients who applied to Research and Training Hospital of Istanbul Kanuni Sultan Süleyman for endoscopy after the ethics committee approval numbered 19.12.2019 / 056 was obtained. Patients with signs of tumor in the upper gastrointestinal system, who underwent surgery or percutaneous endoscopic gastrostomy, or under 18 years of age were excluded from the study. Statistical analysis SPSS Statistics 22 (IBM Corp. Turkey) program was used and $\mathrm{p}<0.05$ was considered significant.

Results: There was no statistically significant relationship between BMI of the patients and the anatomical localizations of the esophageal hiatus and esophagogastric junction. The esophageal hiatus and esophagogastric junction localizations of male patients were found at a statistically greater distance than women $(\mathrm{p}<0.001)$. While no significant relationship was found between the age of the patients and the anatomical location of the esophageal hiatus $(\mathrm{p}=0.062)$, a reverse relationship was found between the esophagogastric junction localization ( $\mathrm{p}=0.045)$. Although there was no significant relationship between BMI and esophageal hiatus and esophagogastric junction localizations, a significant correlation in the same direction was found between both height and weight and both anatomic locations $(\mathrm{p}<0.001)$.

Conclusion: As a result of the study, it can be said that BMI values and esophageal hiatus and esophagogastric junction localizations may change depending on height and weight. We think that the relationship between these differences and diseases may be a separate research topic. In addition, this study is a preliminary study and continues to research by increasing the number of patients.

Keywords: endoscopy, esophageal hiatus, esophagogastric junction, body mass index

\section{P-17}

\section{Acromial curvature types and morphometric study}

Kiran H, Ortadeveci A, Özden H

Department of Anatomy, Faculty of Medicine, Eskişehir Osmangazi University, Eskişehir, Turkey

Objective: Acromion is the posterolateral extension of spina scapulae and curls towards the anterior, joining with the clavicula. Different forms of acromion are associated with various pathologies, such as impingement syndrome and rotator cuff tear. Rotator cuff tears are one of the most common ailments in the shoulder; While its specific etiology is not fully known, it has been suggested that it is due to the combination of intrinsic and extrinsic factors. Recent studies have highlighted specific 
acromion morphologies that were prone to this. The hooked acromion is a frequent contributor to soft-tissue disorders such as subacromial bursitis, shoulder impingement, and rotator cuff dysfunction. In this study, acromion morphometric measurements and classifications were carried out taking into account the importance of acromial curvature in clinical practice.

Methods: A total of 21 scapulae which 7 of them were right, 14 of them were left; with an unknown age and gender were studied in the anatomy laboratory of Eskişehir Osmangazi University. Length and width of processus acromialis, length of scapula, coracoacromial and glenoacromial distances were measured using a digital caliper. Acromial tilt angle was measured and the types were classified as type I $\left(<13^{\circ}\right)$, type II $\left(13-27^{\circ}\right)$ and type III $\left(>27^{\circ}\right)$.

Results: The mean length and width of processus acromialis is 4.75 and $2.36 \mathrm{~cm}$; The mean length of the scapula was measured as $15.04 \mathrm{~cm}$. Mean coracoacromial and glenoacromial distances are noted as $3.74 \mathrm{~cm}$ and $2.82 \mathrm{~cm}$. The most commonly encountered shape of acromion is Type II - Curved (61.90\%); whilst Type I- Flat and Type III- Hooked are equally least encountered shapes $(19.05 \%)$. The mean angle of the acromial curve was $9.25^{\circ}$ for Type I, $17.69^{\circ}$ for Type II and $35.5^{\circ}$ for Type III. The mean coracoacromial distance was measured as $4.13 \mathrm{~cm}$ in Type I, $3.80 \mathrm{~cm}$ in Type II and $3.15 \mathrm{~cm}$ in Type III in our study.

Conclusion: The acromion types and their frequency is important for the orthopedic surgeons who work in this area. Scapulae were typed according to their acromial curvatures and morphometric measurements were made in order to understand the anatomy of the region more comprehensively and to be a guide in understanding and treating shoulder joint disorders.

Keywords: acromion, scapula, acromial curvature, rotator cuff tear, impingement syndrome, morphometric study

\section{P-18}

Morphometric measurements of the anatomical structures of the humerus

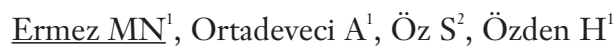

'Department of Anatomy, Faculty of Medicine, Eskişehir Osmangazi University, Eskişehir, Turkey; 'Health Services Vocational School, Eskişehir Osmangazi University, Eskişehir, Turkey

Objective: The humerus, also called the arm bone, is the thickest and longest bone of the upper limb. Dry bones with various grooves, rough areas and other anatomical structures provide information about the localization of adjacent structures such as muscles, nerves and thus provide useful information for orthopedic diseases that are frequently encountered in the clinic. In this study, in addition to general morphometric measurements such as the total length of the bone and the circumference at different levels, we measured some special structures such as radial groove and deltoid tuberosity which provide information about muscle and nerve neighborhood.
Methods: This study was conducted on 55 undamaged humerus bones (34 left, 21 right) in Eskişehir Osmangazi University Medical Faculty Department of Anatomy Laboratory. Digital calipers, tape measure and ruler are used as measurement tools. The length of the bone, the distance between the anatomical neck and the surgical neck medially, the circumference of the surgical neck, the circumference of the midpoint of deltoid tuberosity and its distance to the top of the bone, the distance between the radial groove to the most protruding point of the capitulum in front, the distance between the epicondyles, total transversal length of the capitulum and the trochlea, widths and heights of radial fossa - coronoid fossa and olecranon fossa were measured and their averages were calculated.

Results: According to the results of this study, the average humerus length for all bones was found to be $30.82 \pm 1.98 \mathrm{~cm}$. While the mean circumference of the surgical neck was $8.02 \pm 0.71 \mathrm{~cm}$, the mean distance between surgical neck and anatomical neck medially was measured as $1.85 \pm 0.27 \mathrm{~cm}$. The mean circumference of the bone at the midpoint of deltoid tuberosity was $6.67 \pm 0.69 \mathrm{~cm}$, and the mean distance of deltoid tuberosity to the top of the bone was calculated as $12.61 \pm 1.08$ $\mathrm{cm}$. The mean distance between the radial groove to the most protruding point of the capitulum in front was calculated as $17.55 \pm 1.67 \mathrm{~cm}$. The mean width and height of coronoid fossa were $1.44 \pm 0.24 \mathrm{~cm}$ and $0.99 \pm 0.30 \mathrm{~cm}$, respectively, while the mean width and height of radial fossa were $1.18 \pm 0.18 \mathrm{~cm}$ and $0.67 \pm 0.21 \mathrm{~cm}$. The mean width and height of olecranon fossa were calculated as $2.31 \pm 0.27 \mathrm{~cm}$ and $1.21 \pm 0.28 \mathrm{~cm}$. The mean distance between the medial epicondyle and the lateral epicondyle was measured as $6.07 \pm 0.42 \mathrm{~cm}$.

Conclusion: The humerus is an extremely important bone in terms of its structures and connections. It is crucial for the clinicians who will work in this region to have full knowledge on anatomy of the region in terms of successful treatment/intervention. In this context, obtaining detailed information about certain bone structures and the course of neurovascular structures on the bone is of great importance for clinicians.

Keywords: humerus, morphometry, radial groove, deltoid tuberosity

\section{P-19}

M. extensor pollicis longus incision recognized later in the healed extensor sheath after hand injury: a case report

Canbay Durmaz $\mathrm{S}^{1}$, Özbağ D ${ }^{2}$, Solgun $\mathrm{S}^{1}$, Canbay A

'Department of Anatomy, Institute of Health Sciences, Inönü University, Malatya, Turkey; '2Department of Anatomy, Faculty of Medicine, Inönü University, Malatya, Turkey; ${ }^{3}$ Department of Orthopedics and Traumatology, Elbistan State Hospital, Kahramanmaraş, Turkey

Objective: To emphasize the importance of checking the anatomical continuity of the tendon, even in the presence of an 
intact extensor sheath in surgical interventions, and not overlooking potential tendon damage by evaluating the muscle activity in detail in traumatic hand injuries.

Case: A 21-year-old male patient admitted to the emergency room with laceration in his hand after a knife injury was discharged with superficial stitches on the skin. The patient found it appropriate to consult a physiotherapist due to the limitation of thumb movements after a few days. As a result of the evaluations, the patient was referred to the orthopedic polyclinic, thinking that there would be an m. extensor pollicis longus laceration or rupture. In the surgical procedure performed 1 week later, it was observed that the $\mathrm{m}$. extensor pollicis longus tendon was completely ruptured and the cut tendon ends were distanced from each other without any scar and adhesion. In addition, the presence of a healed or intact extensor sheath is an extremely rare condition. In surgery, the tendons were stitched end to end, and the patient was referred to physiotherapy again.

Conclusion: Hands are among the organs used a lot in daily life activities and can therefore be exposed to various traumas. It is very important to know the anatomical structure well in accurately evaluating the structures damaged during injury. Incorrect examinations sometimes cause irreversible loss of function. In our case, the presence of a tendon cut detected late revealed an intact extensor sheath even if it is seen rarely. This intact sheath may cause mistakes especially in surgeries. It should be considered that there may be a self-repairing sheath or a structure that remains intact, as it does after spontaneous tendon ruptures, as in our case, tendon continuity should be examined and the possibility of a potential rupture or laceration should not be ignored.

Keywords: m. extensor pollicis longus, rupture, extensor sheath, hand surgery

\section{P-20}

A case of isolated unilateral absence of the pulmonary artery

$\underline{\text { Gökşan AS }}{ }^{1}$, Digilli B ${ }^{1}$, Açar $\mathrm{G}^{1}$, Şahin $\mathrm{O}^{2}$

'Department of Anatomy, Meram Faculty of Medicine, Necmettin Erbakan University, Konya, Turkey; ${ }^{2}$ Department of Nuclear Medicine, Meram Faculty of Medicine, Necmettin Erbakan University, Konya, Turkey

Objective: Unilateral absence of the pulmonary artery (UAPA) is a congenital malformation first reported by Fraentzel in 1868 . UAPA is a rare anomaly that usually occurs with other cardiovascular malformations such as tetralogy of Fallot, ventricular septal defect, transposition of great vessels and aortic arch anomalies. Although respiratory symptoms such as dyspnea or hemoptysis may be seen, approximately $30 \%$ of UAPA patients are asymptomatic. In addition, isolated absence of the right pulmonary artery has been associated with pulmonary hypertension (PHT) in $19-44 \%$ of patients in different studies. The diagnosis of UAPA can be made by chest radiography, echocar- diography, CT and MRI angiography and angiography plays an important role in detecting collateral circulation and choosing the treatment. Scintigraphy is also a method used for the diagnosis of UAPA, to see the perfusion status of the lungs and to plan surgical treatment. The aim of this study is to review an isolated unilateral absence of pulmonary artery case with radiological imaging.

Methods: Necmettin Erbakan University Meram Faculty of Medicine, Department of Nuclear Medicine, computed tomography (CT), magnetic resonance (MRI) angiography and ventilation-perfusion scintigraphy (V/Q) image reports and literature review.

Results: Isolated absence of right pulmonary artery was detected in computed tomography (CT) and magnetic resonance (MRI) pulmonary angiography images in a 58-year-old female patient. In addition, no perfusion was observed in the right lung on scintigraphy images.

Conclusion: In previous studies, it has been reported that the methods such as pneumonectomy, lobectomy and selective embolization have been used in the surgical treatment of UAPA, and medical therapy has been shown to improve patients with PHT with exertional dyspnea. Hemoptysis and exertional dyspnea are the most common symptoms in patients with UAPA. So, UAPA should definitely be considered in the differential diagnosis of patients with unexplained hemoptysis and exertional dyspnea. Also, early diagnosis and management of isolated UAPA is very important to prevent the devastating effect of massive hemoptysis or severe PHT in the long term.

Keywords: absence of pulmonary artery, CT angiography, MRI angiography, pulmonary hypertension, pulmonary scintigraphy

\section{P-21}

Two rare cases of herniated urinary bladder in the inguinal canal and its clinical importance

$\underline{\text { Ilgin } \mathrm{F}^{1}}$, Açar $\mathrm{G}^{1}$, Şahin $\mathrm{O}^{2}$, Çiçekçibaşı $\mathrm{AE}^{1}$

'Department of Anatomy, Meram Faculty of Medicine, Necmettin Erbakan University, Konya, Turkey; ${ }^{2}$ Department of Nuclear Medicine, Meram Faculty of Medicine, Necmettin Erbakan University, Konya, Turkey

Objective: Herniation of the bladder into the inguinal canal accounts for less than $5 \%$ of all inguinal hernias. It is more common over the age of 50 and on the right. Although mostly asymptomatic, lower urinary tract symptoms such as scrotal swelling, urgency, and nocturia can be seen. It may cause complaints such as urination reinforcement by pressing on the abdomen and swelling area and at regular intervals urination. In more severe cases, hydronephrosis may also present with a more complex clinical picture such as hematuria and acute renal failure. It is usually detected incidentally and intraoperatively. Diagnosis before inguinal hernia surgery is important in terms 
of preventing possible complications. Less than $7 \%$ of cases are diagnosed preoperatively. The aim of this study is to present the rare case of inguinoscrotal hernia and to emphasize that it may be found in the hernia sac.

Methods: Literature review and 18F-fluorodeoxyglucose positron-emission tomography/computed tomography (FDG PET/CT) images in the archives of the Department of Nuclear Medicine were scanned retrospectively and two rare cases of herniated urinary bladder in the inguinal canal were observed.

Results: Inguinoscrotal bladder herniation was detected in PET examinations of a 72-year-old male patient with a body weight of $92 \mathrm{~kg}$ and another 79-year-old male patient with a body weight of $82 \mathrm{~kg}$.

Conclusion: Considering the severity of the complications, the importance of radiological diagnosis before the operation increases. If it is not known that the bladder is the pathology that causes the hernia during inguinal hernia surgery or the bladder is in the hernia sac, it may cause bladder injuries. As a result, it should not be forgotten that the bladder may herniate into the inguinal canal, and the diagnosis of inguinoscrotal bladder herniation before the operation is important in terms of preventing complications.

Keywords: bladder, inguinoscrotal hernia, PET CT, urinary system

\section{P-22}

The case of elastofibroma dorsi and its clinical importance

Şeker B ${ }^{1}$, Açar $\mathrm{G}^{1}$, Çiçekcibaşı AE ${ }^{1}$, Kaya B ${ }^{2}$

'Department of Anatomy, Meram Faculty of Medicine, Necmettin Erbakan University, Konya, Turkey; '2Department of Nuclear Medicine, Meram Faculty of Medicine, Necmettin Erbakan University, Konya, Turkey

Objective: Elastofibroma dorsi (ED) is a benign soft tissue tumor. It is characterized by slow-growing fibroelastic tissue proliferation and accumulation of abnormal elastic fibers in adipose tissue stroma. This tumor is typically located deep within the lower end of the scapula. They are relatively common lesions and often occur in the elderly and women. It is bilateral in $10 \%$ of cases. Symptomatic cases can be found in shoulder pain with a growing soft tissue mass, locking and mass sounding sounds of soft tissue mass due to the movement of the scapula, but their symptoms are not specific. Therefore, it can often be confused with rotator cuff and cervical spine pathologies. It is usually asymptomatic and often diagnosed after an accidental discovery. Characteristic findings in ultrasonography (US), computerized tomography (CT) and magnetic resonance imaging (MRI) are generally sufficient for diagnosis. In addition, ED can show uptake of $18 \mathrm{~F}$-fluorodeoxyglucose (18F-FDG) in positron emission tomography (PET / CT) combined with CT. The aim of this study is to review the case of elastofibroma dorsi with PET / CT findings in addition to radiological CT findings.

Methods: Literature review and 18F-FDG PET/CT images were scanned retrospectively in the archives of the Department of Radiology.

Results: Elastofibroma dorsi showing FDG uptake in the subscapular region and detected as fluid density on CT was observed in two female patients aged 70 years, $65 \mathrm{~kg}$ and 64 years old, $85 \mathrm{~kg}$.

Conclusion: Elastofibroma dorsi is usually diagnosed incidentally, as it is asymptomatic in approximately $50 \%$ of cases. In addition, in symptomatic cases, the symptoms are not specific. So it can be confused with other pathologies. To avoid misdiagnosis, it is important to know PET / CT findings in addition to US, CT and MRI for correct diagnosis.

Keywords: elastofibroma dorsi, computerized tomography, FDG uptake, positron emission tomography, soft tissue tumor

\section{P-23}

\section{A case of bilateral double renal artery}

$\underline{\text { Admıs } H^{1}}$, Açar $\mathrm{G}^{1}$, Çiçekcibaşı AE ${ }^{1}$, Koplay $\mathrm{M}^{2}$

${ }^{\prime}$ Department of Anatomy, Meram Faculty of Medicine, Necmettin Erbakan University, Konya, Turkey; ${ }^{2}$ Department of Radiology, Faculty of Medicine, Selçuk University, Konya, Turkey

Objective: Arterial blood supply of the kidneys is provided by one renal artery separated from the lateral faces of the abdominal aorta on both sides. Renal arteries originate from the abdominal aorta at the level of the intervertebral disc between the first and the second lumbar vertebrae. Normally, there is one renal artery for each kidney and generally the renal artery divides into the ramus anterior and ramus posterior branches close to the hilum renale. Since its embryological development shows complexity, variations of renal artery are very common. The variations of renal artery are divided into two groups: early branching and extra renal artery. In the literature, it has been reported that there are 5 types of variation of extra renal artery in classical classification: Type I: Normal renal artery; Type II: Double renal arteries on the right (the most common variation); Type III: Double renal arteries on the left Type IV: Three renal arteries on the left; Type V: Bilateral double renal arteries.

Methods: Reports of abdominal computed tomography angiography (CTA) images of Selçuk University Faculty of Medicine Department of Radiology and literature review.

Results: Bilateral double renal artery (Type V) was identified in a 71-year-old male patient on retrospective CTA images.

Conclusion: Knowledge of morphology of abdominal aorta and its branches is important in regards to renal transplantation, renal trauma surgery, radiological imaging and surgical treatment of aortic aneurysms.

Keywords: renal artery, computerized tomography angiography, variation, renal transplantation 


\section{P-24}

Categorization of philtrum and nostril with three-dimensional imaging device in Turkish individuals of young adults

\section{Maral F ${ }^{1}$, Keskin $\mathrm{M}^{2}$, Arifoğlu $\mathrm{Y}^{1}$}

'Department of Anatomy, Faculty of Medicine, Bezmialem Vakıf University, Istanbul, Turkey; 'Department of Plastic and Reconstructive Surgery, Faculty of Medicine, Istanbul Medipol University, Istanbul, Turkey

Objective: Although the nasolabial area plays a dominant role in facial aesthetics due to its location in the midline of the face, it has quite variant morphometric measurements due to factors such as age, gender, ethnic origin and geographical climate. Therefore, it reduces the desired level of success in surgical operations performed on the lip-nose complex. Knowing the types of nostrils (Nostril = nares), the shape and morphology of philtrum, whose functional significance has not been fully revealed, is of aesthetic importance to create a natural and symmetrical image of the upper lip in cleft lip surgery. Threedimensional imaging technologies have developed rapidly in the last 10 years, and these developments allow us to reach rapid and effective results in clinical studies. Much work has not been done in the literature on the evaluation of this region with $3 \mathrm{D}$ methods. For this reason, it is aimed to evaluate philtrum and nostril in young adult Turkish individuals more precisely and safely by using advanced technological devices.

Methods: This study, whose ethics committee approval was obtained: was planned prospectively and three-dimensional images of 126 individuals between the ages of 18 and 29 who had no history of craniofacial surgery were taken with the Vectra 3D photosystem (Canfield, Fairfield, NJ) device. The philtrum and nostrils (nostril) were categorized by using certain reference points on the face of the subjects included in the study. The data analysis of the study was performed using the IBM Statistical Package for Social Sciences (SPSS) Version 22.0 (SPSS inc., Chicago, IL, USA) package statistics program. All data obtained were compared between genders.

Results: In this study; the philtrum types are evaluated and divided into 4 categories: triangular, parallel, concave and flat. In a total of 53 people (42.10\%), 22 males and 31 females, parallel type philtrum; in 40 people (31.7\%), 21 males and 19 females, triangular type philtrum; in 23 people (18.3\%), 15 males and 8 females, concave type philtrum and in 10 people (7.9\%), 5 males and 5 females, flat type philtrum was observed. In this study the nostril types were also evaluated and divided into 4 categories: tear-shaped, triangular, round and heartshaped. In a total of 60 people $(47.60 \%), 32$ males and 28 females, tear-shaped nostril; in 44 people (34.90\%), 18 males and 26 females, triangular type nostril; in 13 people $(10.30 \%)$, 7 males and 6 females, round type nostril; in 9 people $(7.10 \%)$, 6 males and 3 females, heart-shaped nostril was observed.

Conclusion: This work gives information about philtrum and nostril types according to gender in young adults in Turkish population. We anticipate that the results we reported in our study will contribute to the literature on this subject and will be a useful guide in Plastic Reconstructive and Aesthetic Surgery to achieve optimum success in the nasolabial area and in the postoperative period.

Keywords: nasolabial evaluation, nostril, nares, philtrum, three-dimensional imaging

\section{P-25}

\section{Variations of the hook of hamate}

Candır BN, Coşkun Ö, Temizsoy Korkmaz F, Gürses IA

Department of Anatomy, Istanbul Faculty of Medicine, Istanbul University, Istanbul, Turkey

Objective: The hook of hamate $(\mathrm{HOH})$ is an important structure that serves as the medial attachment for the transverse carpal ligament and separates the carpal and ulnar tunnels. Additionally, it is used as a common landmark in hand surgery because it is easy to palpate under the skin. It is known that cases with $\mathrm{HOH}$ variations are associated with a higher incidence for Carpal Tunnel Syndrome. Therefore, this study aimed to investigate the morphology and morphometry of the $\mathrm{HOH}$.

Methods: Morphologic and morphometric evaluation of 52 hamates, without gender and age data, from the osteological collection at the Istanbul Faculty of Medicine were performed. The heights of the hamate and the $\mathrm{HOH}$ were measured and the hook/hamate ratio was calculated. The width of the $\mathrm{HOH}$ at its base, waist, and tip was measured. All measurements were performed with a digital caliper. SPSS v.21 was used for statistical analysis.

Results: The $\mathrm{HOH}$ was curved in $35(67.3 \%)$ and flat in 17 (32.7\%) specimens. Average hamate and $\mathrm{HOH}$ heights were $21.97 \pm 2.0 \mathrm{~mm}$ and $10.21 \pm 1.6 \mathrm{~mm}$, respectively. Average hook/hamate ratio was $0.47 \pm 0.06$. Specimens with a hook/hamate ratio less than two standard deviations of the average $(<0.33)$ were considered as hypoplastic $(n=2,3.8 \%)$. Average width of the $\mathrm{HOH}$ at the base, waist, and tip were $8.89 \pm 0.9 \mathrm{~mm}, 8.53 \pm 1.0 \mathrm{~mm}$, and $9.44 \pm 1.68 \mathrm{~mm}$, respectively. Parametric variables did not show significant difference between right and left sides ( $>00.05)$. Similarly the hamate and $\mathrm{HOH}$ heights were not different for curved and flat $\mathrm{HOH}$ ( $>0.005)$. Nevertheless, base (9.1 vs $8.5 \mathrm{~mm}$; $\mathrm{p}=0.33$ ), waist ( 8.8 vs $8 \mathrm{~mm}$; $\mathrm{p}=0.007)$, and tip (9.9 vs $8.4 \mathrm{~mm}$; $\mathrm{p}=0.008$ ) widths were significantly larger for curved $\mathrm{HOH}$.

Conclusion: Identifying a $\mathrm{HOH}$ fracture is troublesome in conventional radiologic and ultrasonographic evaluations. Undiagnosed $\mathrm{HOH}$ fractures have the risk to injure the ulnar nerve and artery along with flexor tendons. Therefore, knowing the normal and variable anatomy of the $\mathrm{HOH}$ gains importance for radiologists and hand surgeons. This study showed 
that the variations of the $\mathrm{HOH}$ were compatible with the literature. Nevertheless, relative small sample size and having no bilateral specimens limited the statistical analysis. Additionally, expanding the study with patient data in order to investigate the relationship between $\mathrm{HOH}$ morphology and $\mathrm{HOH}$ fractures was planned.

Keywords: hamate, hook of hamate, fracture, variation

\section{P-26}

Estimating the localization of the jugular foramen using important anatomical landmarks in the region

Güner Sak N ${ }^{1}$, Tuncel Çini $N^{1}$, Babacan $S^{2}$, Şendemir $E^{1}$

'Department of Anatomy, Faculty of Medicine, Bursa Uludağ University, Bursa, Turkey; ${ }^{2}$ Department of Anatomy, Faculty of Medicine, Harran University, Şanlıurfa, Turkey

Objective: The jugular foramen is a passage for numerous neurovascular structures that have clinical importance. The anteromedial compartment (nervous part) contains the glossopharyngeal nerve and the inferior petrosal sinus. The posterolateral compartment (vascular part) contains the jugular bulb, the vagus nerve, and the spinal portion of the accessory nerve. Multiple surgical approaches and combinations have been described to gain access to the jugular foramen. In this area, the best surgical approach for the treatment of rare pathologies must be taken into consideration for the protection of the important structures of the region. This study aims to predict the localization of the jugular foramen by calculating formulas using morphometric measurements with the neighboring important anatomical structures of the skull.

Methods: The study was carried out on 25 dry skulls in the laboratory of the Department of Anatomy, Faculty of Medicine, Bursa Uludağ University, and Department of Anatomy, Faculty of Medicine, Harran University. A sliding caliper was used for measurements on dry bones. A total of 17 parameters were evaluated in the study by using these parameters formulas will be calculated. Statistical analyzes were performed by SPSS 22.0.

Results: The regression formulas were developed in order to estimate jugular foramen localization using anatomical reference points in the region. The formulas such as; the distance between the posterior edge of jugular foramen- the posterior root of zygomatic arch $=2.154+(8.77 \mathrm{x}$ the distance between the posterior root of zygomatic arch - the mastoid process) Adjusted R2=0.687; $\mathrm{SE}=2.701$. The distance between the posterior edge of jugular foramen - the digastric point) $=41.863$ ( $0.492 \mathrm{x}$ the distance between the digastric point) - the asterion) Adjusted R2=0.484; $\mathrm{SE}=2.821$

Conclusion: Estimating the localization of the jugular foramen using important landmarks is significant in the surgical procedures to be performed in this region; therefore, we think this information will help clinicians working in this area.
Keywords: skull, jugular foramen, asterion, digastric groove, mastoid process

\section{P-27}

Three headed biceps brachii

Kızılay Ö

Department of Anatomy, Faculty of Medicine, Aydin Adnan Menderes University, Aydın, Turkey

Objective: Musculus biceps brachii is one of the muscles that shows frequent variations. Most common variation is that it has extra heads. The most common of these variations is the threeheaded musculus biceps brachii. To the best of our knowledge, a literature review has not been conducted for the years 1880-2020 on the triple-headed musculus biceps brachii. Another issue that draws our attention is that a common classification is not used in case reports. With this study, we aim to contribute to the literature on the two issues mentioned above.

Methods: Pubmed search engine was used to collect data in our study. More than two hundred articles were searched by entering the keywords: three headed biceps brachii, third head of biceps brachii, atypical biceps brachii, supernumerary head of the biceps brachii and accessory head of biceps brachii into the pubmed search engine. Among these articles, those that are only in summary, male / female, right / left extremity, origo / insertio data missing or not specified and those not suitable for the topic of three-headed biceps brachii were not included in the study. During the classification of the origin of the third head of musculus biceps brachii, the method of Marc RodriguezNiedenführ and colleagues was applied.

Results: Three-headed musculus biceps brachii was seen with a rate of $78.6 \%$ in men and $21.4 \%$ in women. When all the right and left extremities were evaluated, $70.5 \%$ infero-medial and $29.4 \%$ superior humeral head were detected. No infero-lateral humeral head was found. No statistical significance was found between origo's being in the infero-medial or superior humeral region and gender ( $\mathrm{p}=0.196$ for the right upper extremity; $\mathrm{p}=0.077$ for the left upper limb). In addition, when articles mentioning nervus musculocutaneus were examined, it was mentioned that the nervus musculocutaneus was absent, duplicated, not piercing musculus coracobrachialis and frequently exhibiting a connecting branch.

Conclusion: Having a third head of musculus biceps brachii can cause shoulder pain, nerve and vascular entrapment, and / or variations. May cause confusion in surgical procedures performed in the shoulder area. We think that our research will increase awareness about the frequency of the third head of musculus biceps brachii.

Keywords: musculocutaneous nerve, variation, biceps brachii, third head 


\section{P-28}

\section{Duplication of inferior vena cava: case report}

$\underline{\text { Akdoğan } S^{1}}$, Kadiyoran $\mathrm{C}^{2}$, Yilmaz MT ${ }^{1}$

'Department of Anatomy, Meram Faculty of Medicine, Necmettin Erbakan University, Konya, Turkey; ${ }^{2}$ Department of Radiology, Meram Faculty of Medicine, Necmettin Erbakan University, Konya, Turkey

Objective: Inferior vena cava (IVC) duplication is a rare anomaly. Especially during the daily practice of radiologists and vascular surgeons; it is important in retroperitoneal surgery and in the treatment of thromboembolism. Anatomical variations may sometimes occur during embryogenesis, which is a complex structuring. The prevalence of these variations is around $0.2-4 \%$ in the population. The most important risk of these variations is a high risk of developing deep vein thrombosis in femoral and common iliac veins at young ages. These variations are especially important during surgical interventions. Location definitions should be made very carefully, especially in laparoscopic donor nephrectomy.

Case: A double IVC was detected on both sides of the abdominal aorta in the CT examination of a 41-year-old male patient who applied to the emergency department of N.E.Ü Meram Medical Faculty with the complaint of diffuse abdominal pain. This phenomenon is defined as double IVC in the literature. It was determined that the left IVC opened directly into the right IVC and then drained into the right atrium as a single vessel. Apart from this, no renal vein and kidney anomalies were observed.

Conclusion: IVC and the development of its major branches is a multi-layered process in the form of main stages characterized by progression followed by regression of venous structures. In some periods, this process is not shaped as it is conveyed and anomalies develop. These anomalies are asymptomatic with clinical consequences and are diagnosed on routine imaging studies. IVC develops from subcardinal, supracardinal and posterior cardinal vessels. Both posterior cardinal veins join to form the iliac veins and their junction. The right supracardinal vein turns into infrarenal IVC and the left supracardinal vein regresses normally. Right subcardinal - supracardinal anastomosis develops in the renal part of the IVC and the right subcardinal veins along with the hepatic vein develop towards the rest of the IVC. Duplication is caused by the persistence of the supracardinal veins on both sides and forms the right supracardinal veins and morphologically double IVC. Almost all vascular anomalies are detected incidentally by CT or magnetic resonance imaging, and congenital anomalies of the IVC are often seen in asymptomatic patients. In double IVC cases, right and left IVCs may be of equal diameter or one larger than the other. Left IVC typically ends at the left renal vein level. Left IVC drains first into the left renal vein and then they drain into the right IVC together. Double IVC should be suspected in case of an embolism attack that occurs despite filter placement.
Conclusion: IVC duplication is a rare but important anomaly. Most cases are diagnosed by chance. However, this venous anomaly has important clinical implications, especially during retroperitoneal surgery and venous interventional radiology. Therefore, it is vital for surgeons to be familiar with these anomalies to reduce the risk of serious bleeding during surgical treatment and to prevent operative complications.

Keywords: inferior vena cava, anatomy, variation, duplication

\section{P-29}

A case with hypogenesis of the corpus callosum detected by magnetic resonance imaging method

Gürlek Celik N ${ }^{1}$, Cebeci $\mathrm{H}^{2}$, Karabulut $\mathrm{AK}^{3}$, Fazlıŏulları $\mathrm{Z}^{3}$

'Faculty of Health Sciences, Ahi Evran University, Kırşehir, Turkey; ${ }^{2}$ Department of Radiology, Faculty of Medicine, Selçuk University, Konya, Turkey; ${ }^{3}$ Department of Anatomy, Faculty of Medicine, Selçuk University, Konya, Turkey

Objective: Corpus callosum is the largest of commissural pathways connecting the same centers of both hemispheres. Corpus callosum, which consists of 200-250 million axons, is responsible for information exchange and coordination by creating connections between similar centers in the hemispheres. Corpus callosum, which located at the base of fissura sagittalis süperior and covering the upper part of ventriculus lateralis, consists of 4 sections from front to back; rostrum corporis callosi, genu corporis callosi, truncus corporis callosi and splenium corporis callosi. Developmental anomalies such as agenesis, hypogenesis, hypoplasia may occur in the corpus callosum as a result of various factors. The purpose of this study is to evaluate magnetic resonance imaging (MRI) findings of the hypogenesis of the corpus callosum.

Case: Sagittal and axial sections and MRI findings of a 23-yearold male who applied to Selçuk University Medical Faculty were evaluated. Hypogenesis was detected in the rostrum corpus callosi and splenium corporis callosi of the corpus callosum in T1 sagittal and T2A axial sections.

Conclusion: Hypogenesis of the corpus callosum is also defined as partial agenesis. Hypogenesis may occur with the absence of commissural pathways. Corpus callosum develops from ventral to dorsal with the formation of massa commissuralis between the 6th and 20th weeks of pregnancy. During the development, conditions such as genetic factors, radiation, nutrition and hypoxia may cause impairment in the crossing of callosal fibrils. Classification of the anomalies resulting from this impairment can be easily detected with MRI. Due to the presence of many congenital brain anomalies accompanying corpus callosum anomalies, we think that patients with neurological problems should be examined with MRI to clarify the diagnosis of the disease.

Keywords: corpus callosum, hypogenesis, magnetic resonance imaging, embryology 


\section{P-30}

Unilateral extraorbital muscle involvement in graves ophthalmopathy: a case report

Yilmaz NA ${ }^{1}$, Kadiyoran $C^{2}$, Yilmaz MT ${ }^{3}$

'Faculty of Health Sciences, Necmettin Erbakan University, Konya, Turkey; ${ }^{2}$ Department of Radiology, Meram Medical Faculty, Necmettin Erbakan University, Konya, Turkey; ${ }^{3}$ Department of Anatomy, Meram Medical Faculty, Necmettin Erbakan University Konya, Turkey

Objective: Graves' disease is an autoimmune disease characterized by hyperthyroidism, diffuse goiter, ophthalmopathy and rarely dermopathy. Graves Ophthalmopathy (GO) is one of the non-thyroid findings of Graves disease and is known as Graves orbitopathy, thyroid eye disease or ophthalmopathy accompanying thyroid. While $10-50 \%$ of patients with Graves have clinically ophthalmopathy, it can be detected in approximately $100 \%$ when imaging techniques are used. In this study, involvement of extraocular muscles in unilateral graves ophthalmopathy is discussed.

Case: 35-year-old male patient in September 2020 to the ophthalmology clinic; presented with complaints of dry left eye, eye movement disorder, eyelid retraction. After the anamnesis, it was determined that her complaints about her eye started three years ago and she was treated for glaucoma suspicion, and she was operated on with the diagnosis of Graves in 2019. After contrast Orbital MRI examination, left $\mathrm{m}$. rectus medialis (10 $\mathrm{mm}), \mathrm{m}$. rectus inferior $(9.4 \mathrm{~mm}), \mathrm{m}$. rectus superior $(9.4 \mathrm{~mm})$ and $\mathrm{m}$. rectus lateralis $(7.4 \mathrm{~mm})$ showed a significant increase in muscle thickness and volume compared to the right side. The tendons were unaffected.

Conclusion: Generally, GO is bilateral and rarely unilateral. The frequency rate is slightly higher in women compared to men. In extraocular muscle involvement, unlike myositis, there is expansion in the muscle other than the tendon. Order of frequency in involvement $\mathrm{m}$. rectus Inferior, medialis, superior and rarely lateralis form. In this case, the involvement is one-sided. The intensity of involvement is at most $\mathrm{m}$. rectus medialis, later seen in superior, inferior and least lateralis muscles. Thickness and intensity increase was determined. M. rectus lateralis, which is rarely mentioned in the literature, measured an increase in thickness of $2.6 \mathrm{~mm}$ as an approach. In rare cases, imaging methods are needed for follow-up, treatment and differential diagnosis in unilateral involvement, such as unilateral Graves' ophthalmopathy. It has been found that the involvement of the rectus lateralis muscle should not be ignored.

Keywords: Graves ophthalmopathy, MR, extraocular muscle, musculus rectus lateralis

\section{P-31}

Morphometry of the musculotubal canal on the skulls

Tuncel Çini N, Ari I

Department of Anatomy, Faculty of Medicine, Bursa Uludağ University, Bursa, Turkey
Objective: The eustachian tube (tuba auditiva), also called the pharyngotympanic canal, is a narrow fibrocartilaginous canal that connects the middle ear to the nasopharynx. Structurally, the eustachian tube consists of areas of bone which is named musculotubal canal and cartilage area from lateral to medial. The function of measuring the eustachian tube has become an important part of the preoperative evaluation of myringoplasty and tympanoplasty procedures. The aim of the study is to give descriptive values by performing morphometric measurements on the bone part of the eustachian tube.

Methods: The study was carried out using 26 dry skulls belonging to the bone collection of Bursa Uludağ University Faculty of Medicine, Department of Anatomy. In addition to 12 variables determined on bones for the osseous part of tuba auditiva, cranial index calculation and pterion typing were performed. Measurements were made with Somet Inox sliding caliper and SPSS 22.0 program was used for statistical analysis.

Results: When correlation analyzes were made as a result of the measurements, "the distance between the lateral and medial edges of the right and left musculotubal canal", "the distance between the musculotubal canal and the basis of medial lamina on the right and left", "between the right and left pyramidal process". Significant correlations were found in "distance" and "distance between right and left medial lamina" measurements $(\mathrm{p}<0.05)$. There was no significant relationship between cranial index and pterion types.

Conclusion: Most otologists consider that the eustachian tube obstruction to be a major etiological factor in chronic, suppurative, middle ear disease, and restoration is necessary for a successful outcome. We think that the data obtained will be helpful in interventions to be performed in the region such as eustachian balloon tube dilation. On the other hand, it should not be forgotten that the data can be used in creating threedimensional models of the region.

Keywords: Eustachian tube, skull, morphometry, musculotubal canal

\section{P-32}

\section{Anthropometric evaluation of the femur}

Özcan BN, Akça Andı Z, Uzun A, Emirzeoğlu M

Department of Anatomy, Faculty of Medicine, Ondokuz Mayıs University, Samsun, Turkey

Objective: The objective was to determine the morphometric parameters of proximal and distal femur and to discuss its clinical application.

Methods: The study comprised 67 femurs (33 right, 34 left) which were obtained from the Anatomy Department. Length of femur, length of femoral neck, circumference of femoral neck, femur bicondylar width, intercondylar width, medial/lateral condylar depth, intercondylar depth, femur head diameter, femur neck diameter were measured with using $0,1 \mathrm{~mm}$ preci- 
sion digital vernier caliper, tape measure. The inclination angle was measured with a goniometer. The statistical analysis was performed by SPSS 22.00 with Shapiro-Wilk, Student-T, Mann Whitney U.

Results: According to the results of our study; average femur length was $430.38 \pm 29.04 \mathrm{~mm}$ on the right side and $425.19_{ \pm}$ $31.26 \mathrm{~mm}$ on the left side. Femoral inclination angle was $123.90 \pm 2.24^{\circ}$ on the right side and $123.91 \pm 2.06^{\circ}$ on the left side. Medial condylar depth of the femur was $59.57 \pm 7.97 \mathrm{~mm}$ on the right side and $55.81 \pm 5.80 \mathrm{~mm}$ on the left side. Medial condylar depth of femur was found significantly different right and left sides. ( $\mathrm{p}=0.015$ ) No significant difference was found between the left and right sides in other parameters.

Conclusion: It is important to know the femur morphometry and to predict and know the race, gender and sports activities of the individual. Morphometric values obtained from femur clinically. It is important for the procedures to be performed on the hips, thighs and the region and the prostheses to be used next to it. In addition, the daha obtained can contribute to the biomedical engineering, ergonomics, and forensic science discipline.

Keywords: femur, anthropometry, morphometry

\section{P-33}

Canalis suprascapularis: a new terminologic proposal for suprascapular tunnel

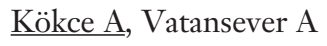

Department of Anatomy, Faculty of Medicine, Balıkesir University, Balıkesir, Turkey

Objective: The main objective of the review was to provide current information about the recently proposed term "canalis suprascapularis" for educational and clinical purposes.

Methods: Literature scanning using Pubmed was completed and reached 57 articles in total. We screened the most up to date and the most related articles to osteofibrous tunnels morphology in this review. Finally 11 of those 57 articles were included.

Results: Most of the studies about suprascapular nerve entrapment is focusing on nerve entrapment within suprascapular and spinoglenoid notches rather than other nerve passage regions. An osteofibrous canal with clearly visible boundaries can be revealed during dissection in supraspinatus fossa but there is no consensus about its terminology that is used for this topographical region. Especially, Al-Redouan et al. 2020, proposed the term "Suprascapular Canal" for this structure and defined its borders. The suprascapular canal can be visualized using a shoulder MRI in the axial plane.

Conclusion: Suprascapular nerve entrapments may occur in other regions besides notches which it passes through. Detailed knowledge of anatomical topography between these structures is crucial for complete education of this region. Moreover, this detailed knowledge could be useful during clinical practices for diagnosis and planning treatment methods against suprascapular nerve entrapments.
Keywords: suprascapular entrapment, suprascapular pain, suprascapular anatomy, fossa supraspinatus

\section{P-34}

Radiological anatomy of extent of breast cancer and the sick lobe hypothesis

Durur Subaşı I ${ }^{1}$, Kapakin $\mathrm{S}^{2}$

'Department of Radiology, Faculty of Medicine, Istanbul Medipol University, Istanbul, Turkey; ${ }^{2}$ Department of Anatomy, Faculty of Medicine, Atatürk University, Erzurum, Turkey

Objective: Multifocal and multicentric breast cancer and morphological-anatomical aspects of the sick lobe hypothesis will be discussed.

Methods: The extent of the disease was determined by evaluating the mammography (MG), ultrasonography (US), magnetic resonance imaging (MRI) examinations of the patients, in terms of the presence and number of suspicious lesions, and the location of the lesions relative to each other.

Results: 58-year-old patient with invasive ductal carcinoma (IDC), multiple suspicious masses on MG, and corresponding USs. The disease was evaluated as multicentric. 46-year-old patient with IDC, transverse fat-suppressed, contrast-enhanced T1-weighted magnetic resonance imaging (MRI) demonstrates multiple suspicious enhancing masses. 29-year-old patient with IDC, multiple suspicious enhancing masses are observed on 3dimensional maximum intensity projection MRI.

Conclusion: The breast is a modified cutaneous gland responsible for lactation. It consists of 15-25 lobes and a branching duct system. Each lactiferous duct opens to the nipple and is associated with many segmental-subsegmental terminal canals and lobules. The lobule and intra-extralobular terminal ducts constitute the terminal ductal lobular unit. This is where milk is produced and breast cancer arises. Some lobes may have a high cancer tendency (sick lobe hypothesis) and a sick lobe may have different stages of breast cancer sequence. The presence of breast cancer in more than one focal area in the same quadrant means multifocal disease, and involvement in more than one quadrant means multicentric disease. In large breasts, when the distance between the suspicious lesions is less or more than 5 $\mathrm{cm}$, multifocal or multicentric disease exist, respectively. The multifocal or multicentric disease is independent of the size of the primary mass and may interfere with breast-conserving approaches. Reshaping the classical multifocal or multicentric disease concept according to the sick lobe hypothesis will prevent inadequate and unnecessary excisions. Especially with an imaging-guided core biopsy, vacuum-assisted biopsy, and largesection histopathology, the extent of the disease will be determined and all lesions can be excised with clean borders with an appropriate surgical approach. MG, breast US, and MRI have a very high spatial resolution. However, they do not separate the lobes. Therefore, a detailed examination of the quadrant with suspicious lesions and other quadrants, evaluation of the 
parenchyma and ducts between the index mass and the nipple, the use of sagittal and coronal, oblique, radial planes and $3 \mathrm{D}$ imaging techniques in addition to the transverse plane, and combining the data of imaging methods will determine the disease extent more accurately.

Keywords: mammography, magnetic resonance imaging, breast, ultrasonography

\section{P-35}

Cadaver embalming models as primary education material in anatomy education

Fidan N, Çetin A

Department of Anatomy, Medical Faculty, Inönü University, Malatya, Turkey

Objective: The process of fixing the cadaver by giving fixatives with appropriate techniques and methods in order to protect the normal tissue and structure is called embalming. With this review, it is to emphasize the different embalming models in cadaver, which has an undisputed place in Anatomy education, which is the primary education material.

Methods: After the discovery of formaldehyde by Alexander Butlerow, German chemist August Wilhelm von Hofman determined the method of producing formaldehyde from methanol. The first use of formaldehyde for tissue fixation by Ferdinand Blum has made a breakthrough in modern embalming technique / model. Although formaldehyde is a good fixative, the formation of drying in the cadaver embalmed with formaldehyde has prompted researchers to research new fixatives. For this, Laskowski developed the Geneva fixation solution formulation consisting of glycerin to protect the cadaver from drying out and phenol to store it. However, there are many cadaver embalming techniques using different chemical formulations including, Spence's cadaver fixing fluid composition, Norville's cadaver fixing fluid composition, Erskine's cadaver fixing fluid composition, Kinnamon's cadaver fixing fluid composition, Kinnamon's cadaver storage solution composition, Larssen's solution's original composition. Many chemical formulations are used, such as Modified Larssen solution composition, Salt water, Alkyd Resin, Thiel solution, Logan solution, Modified Logan solution, Ethanol-Glycerine Method. In the process of cadaver embalming; although solutions are usually injected through femoralis and carotis communis arteries, they can also be injected through other arteries. Detected cadavers can be preserved in formol or acid phenic (phenol) pools. In addition, plastinates can be obtained from cadavers by the standard plasticization method.

Results: Factors considered by anatomists when comparing different embalming techniques / models; the fixatives used, the rate of decomposition of these fixatives in the tissue and the quality of the fixatives. Different formulations of different chemicals such as formaldehyde, phenol, thymol, glycerine and ethanol continue to be used in the cadaver embalming technique / model. In addition to these formulations, thiel, larssen, modified larssen, logan, ethanol-glycerin, saline or liquid soap solutions, alkyd resin method and plastination method are widely used in cadaver detection. It was observed that the plastinated samples kept their shape in the plasticization method and if xylol was also used in this method, the plasticine samples gained a light and flexible structure.

Conclusion: Medical schools require cadaver; to be developed by internationally accepted standards to compare different embalming models that realistically preserve their final form of death. The comparison and quantitative assessment of the tissue quality in the cadaver by the anatomists / embalming agents should be based on experimental procedures. Nowadays, plasticine method after formaldehyde and phenol, alkyd resin method, saline or liquid soap solutions, thiel, larssen, logan, ethanol-glycerine solutions are widely preferred in cadaver enclosures. Plastinates obtained by the standard plasticization method can be used in education for a long time. It is considered that new studies are needed for embalming modeling in cadavers, which is the primary education material in anatomy education.

Keywords: anatomy, cadaver, embalming, fixation

\section{P-36}

\section{Pancreatic lipoma: CT finding}

Keleş A $^{1}$, Yilmaz MT'2 ${ }^{2}$ Kadiyoran $\mathrm{C}^{3}$

'Health Services Vocational School, Karamanoğlu Mehmetbey University, Karaman, Turkey; ${ }^{2}$ Department of Anatomy, Meram Faculty of Medicine, Necmettin Erbakan University, Konya, Turkey; ${ }^{3}$ Department of Radiology, Faculty of Medicine, Selçuk University, Konya, Turkey

Objective: The pancreas is a very important mixed gland in alveolar structure that secretes both internally and externally. It has 4 sections: The head of the pancreas, the neck of the pancreas, the body of the pancreas, the tail of the pancreas. A protrusion extending towards the midline in the lower part of the head of the pancreas is called the uncinate process. Although the majority of benign or malignant benign pancreatic neoplasms arise from pancreatic epithelial cells, mesenchymal tumors can rarely be derived from the connective, lymphatic, vascular and neuronal tissues of the pancreas. Mesenchymal tumors constitute 1-2\% of all pancreatic tumors and are classified according to their histological origin. Various mesenchymal tumors have been reported, including lipoma, teratoma, schwannoma, neurofibroma, lymphoma, and sarcoma. Pancreatic lipomas are rare and usually detected incidentally on imaging methods. Pancreatic lipomas are rare benign mesenchymal neoplasms with stable size and morphology. It is known that lipomas are asymptomatic as long as they are not large in size or cause compression-related findings. A problem may arise when the lipoma needs to be distinguished from other lesions containing adipose tissue (focal fatty infiltration of the pancreas, teratoma, liposarcoma), but in the case of focal fat infiltration, such a distinction will not make a clinical difference, and liposarcoma is usually large $(>5 \mathrm{~cm})$, heterogeneous and it has been reported to be a less frequent lesion. 
Fatty tissues and pancreatic lipomas in other parts of the body are observed in a similar structure on computed tomography and magnetic resonance imaging. The aim of this study is to present a rare case of pancreatic lipoma detected on computed tomography imaging.

Case: A nodular lesion of about 4 centimeters in diameter, localized at the level of the uncinate process of the pancreas of a 59-year-old female patient, was found in the archive of Necmettin Erbakan University Meram Medical Faculty Radiology Department. The lesion was evaluated as pancreatic lipoma due to its small size and homogeneous density, and CT density characteristics matching the adipose tissue. There is superior mesenteric vein in the anterior neighborhood of the lesion, and normal pancreatic tissue in the posterior neighborhood. In the medial neighborhood of the lesion, the superior mesenteric artery was located, and the distance between the artery was approximately 4 millimeters.

Conclusion: Pancreatic lipoma is very rare. Therefore, it is very important to use radiological imaging methods such as CT and MRI for the detection and differential diagnosis of small pancreatic lipomas.

Keywords: pancreas, lipoma, computed tomography

\section{P-37}

A cranium case with variation of sulcus sinus obliquus occipitalis

Onur S, Adigüzel E

Department of Anatomy, School of Medicine, Pamukkale University, Denizli, Turkey

Objective: Sinus occipitalis localization is important for infratentorial (posterior fossa) craniotomies. The shape changes on the bone caused by the variations of the dural sinuses, which are generally detected by radiological studies, are important in terms of emphasizing the anatomy of variation in the medical education. The variation of sulcus sinus occipitalis, which we detected on the occipital bone during our routine laboratory work, is presented to emphasize its importance in both undergraduate anatomy education and neurosurgical interventions.

Case: In the cranium, which is estimated to belong to a woman in terms of forensic medicine criteria, it was found that the sulcus sinus occipitalis was deeper on the right side of the fossa cranii posterior and there was no sulcus sinus transversus. It was observed that the right sulcus sinus occipitalis started $1.5 \mathrm{~cm}$ below the protuberantia occipitalis interna, became prominent in the right posterolateral of the foramen magnum with an oblique course and extended to the foramen jugulare and joined with the sulcus sinus sigmoideus. It was also found that sulcus sinus transversus did not occur on the right side.

Conclusion: In studies conducted on cadavers and living humans in the literature, the variation suitable for the groove described in this case was named oblique sinus occipitalis.
During the basis cranii anatomy training, we propose that using these types of sinus variations in the skull skeletons found in the laboratory and drawing attention to the variation anatomy in student education. At the same time, we attach importance to the variation of the dura sinuses and their reflections on the bone are emphasized in cadaver training for surgery.

Keywords: sulcus sinus occipitalis, variation, oblique sinus occipitalis, basis cranii

\section{P-38}

\section{A rare case of truncus coeliacomesentericus}

Taşpınar $\mathrm{C}^{1}$, Açar $\mathrm{G}^{1}$, Koplay $\mathrm{M}^{2}$

'Department of Anatomy, Meram Faculty of Medicine, Necmettin Erbakan University, Konya, Turkey; ${ }^{2}$ Department of Radiology, Faculty of Medicine, Selçuk University, Konya, Turkey

Objective: The Celiac Trunk (CT) is an arterial trunk originating from the abdominal aorta just below the aortic hiatus, to the branching point of the left gastric artery (LGA), the common hepatic artery (CHA) and the splenic artery (SA). The Superior Mesenteric Artery (SMA) originates separately from the aorta about $1 \mathrm{~cm}$ below the celiac trunk and they take important in the abdominal viscera vascularization. There is a very close anatomical and functional connection between the celiac trunk and the SMA. Celiacomesenteric Trunk (CMT) is one of variations of the CT known as Type IV. The CMT is described as a single common trunk arising from the aorta anditsbranchesincludingthe SMA and at least major branches of the celiac trunk. According to the classification of the CMT types; Type I: Hepato-gastro-spleno-mesenteric trunk (HGSM trunk), Type II: Hepato-spleno-mesenteric trunk with LGA arising from the aorta (HSM trunk+LGA), Type III: Gastro-spleno-mesenteric trunk with CHA arising from the aorta (GSM trunk+CHA), Type IV: hepato-gastro-mesenteric trunk with SA arising from the aorta (HGM trunk+SA), and Type V: Any other variation that meets the above definition of the CMT.

Methods: Reports of abdominal computed tomography angiography (CTA) images of Selçuk University Faculty of Medicine, Department of Radiology and literature review.

Result: The Celiacomesenteric Trunk (HGSM trunk, type I) was observed on CTA images of a 21-year-old female patient.

Conclusion: Knowledge about the spectrum of CMT variations is important for planning surgical or interventional procedures in the upper abdomen. Identification of CMT variations may avoid vascular complications during medical procedures, such as hepatobiliary surgery, pancreatic surgery, gastrectomy and others like transcatheter arterial chemoembolization. Future studies should further evaluate the impact of using the proposed classification system on interventional surgical procedures.

Keywords: celiac trunk, celiacomesenteric trunk, computed tomography angiography, hepatobiliary surgery 


\section{1st National Anatomy Congress 27-29 November 2020, Online, Turkey}

\begin{tabular}{|c|c|}
\hline \multicolumn{2}{|l|}{ A } \\
\hline Abbasov Taşkındere $T$ & O-90 \\
\hline Acar B & $\mathrm{O}-44$ \\
\hline Açar G & $\mathrm{P}-20, \mathrm{P}-21, \mathrm{P}-22, \mathrm{P}-23, \mathrm{P}-38$ \\
\hline Açar Hİ & PL-7, O-11 \\
\hline Açıkgöz AK & $\mathrm{O}-82$ \\
\hline Adanır SS & O-66 \\
\hline Adıgüzel E & $\mathrm{O}-02, \mathrm{O}-35, \mathrm{P}-37$ \\
\hline Admış $\mathrm{H}$ & $\mathrm{P}-23$ \\
\hline Afşar E & O-87 \\
\hline Ağar A & O-87 \\
\hline Akbulut Y & $\mathrm{O}-51$ \\
\hline Akça Andı Z & $\mathrm{O}-81, \mathrm{P}-32$ \\
\hline Akdemir Aktaş H & O-93 \\
\hline Akdoğan S & $\mathrm{P}-28$ \\
\hline Akkaya M & $\mathrm{O}-11$ \\
\hline Akkın SM & PL-9 \\
\hline Akkoç RF & $\mathrm{O}-41$ \\
\hline Aksu F & $\mathrm{O}-41$ \\
\hline Aktar Uğurlu G & O-85 \\
\hline Aktaş GD & $\mathrm{O}-53$ \\
\hline Albay S & PL-12, O-07, O-26 \\
\hline Albayrak Gezer İ & $\mathrm{O}-13$ \\
\hline Alkan E & $\mathrm{O}-64$ \\
\hline Altınoğlu $M$ & O-76 \\
\hline Apaydın N & $\mathrm{O}-83$ \\
\hline Argalı Deniz M & $\mathrm{O}-21$ \\
\hline $\operatorname{Ar} 1 \dot{I}$ & $\mathrm{P}-31$ \\
\hline $\operatorname{Ar} Z$ & $\mathrm{O}-04$ \\
\hline Arifoğlu Y & P-02, P-24 \\
\hline Armağan $\mathrm{K}$ & $\mathrm{O}-25$ \\
\hline Arpacı MF & $\mathrm{O}-21$ \\
\hline Arslanyolu M & $\mathrm{O}-75, \mathrm{O}-84$ \\
\hline Aslan Felek S & O-85 \\
\hline Aslan $M$ & O-87 \\
\hline Aslantürk $\mathrm{O}$ & $\mathrm{O}-42$ \\
\hline Atalar K & $\mathrm{O}-45, \mathrm{O}-65$ \\
\hline Atçeken A & O-67 \\
\hline Ateş Ş & O-78 \\
\hline Avnioğlu S & $\mathrm{O}-52$ \\
\hline Ayazoğlu M & $\mathrm{O}-80, \mathrm{P} 15$ \\
\hline Aydın BK & $\mathrm{O}-12, \mathrm{O}-43$ \\
\hline
\end{tabular}

\begin{tabular}{|c|c|}
\hline Aydın S & $\mathrm{O}-41$ \\
\hline Aytaç G & PL-13, O-06, O-10, P-03 \\
\hline \multicolumn{2}{|l|}{ B } \\
\hline Babacan S & $\mathrm{O}-31, \mathrm{P}-26$ \\
\hline Babayeva R & O-88 \\
\hline Bağcl G & $\mathrm{O}-27$ \\
\hline Bahçelioğlu M & O-09 \\
\hline Bahşi İ & O-66 \\
\hline Balcı A & $\mathrm{O}-83$ \\
\hline Balcıŏglu HA & $\mathrm{O}-32, \mathrm{P}-06$ \\
\hline Barut Ç & $\mathrm{O}-25$ \\
\hline Başaloğlu H & O-91 \\
\hline Batur A & $\mathrm{O}-27$ \\
\hline Bedre O & $\mathrm{P}-10$ \\
\hline Beton $\mathrm{O}$ & P-05 \\
\hline Bilge D & O-76 \\
\hline Bilge $\mathrm{O}$ & Panel-1 \\
\hline $\operatorname{Bir} F$ & $\mathrm{O}-35$ \\
\hline Bobuş Örs A & O-89 \\
\hline Boduç E & $\mathrm{O}-51$ \\
\hline Boracı $\mathrm{H}$ & O-86 \\
\hline Boyan N & $\mathrm{O}-55, \mathrm{O}-57$ \\
\hline Bozdağ E & P-16 \\
\hline Bozkurt M & $\mathrm{O}-11$ \\
\hline Bülbül M & O-87 \\
\hline Büyükertan $M$ & $\mathrm{O}-70$ \\
\hline Büyükgüdük İ & $\mathrm{O}-75, \mathrm{O}-84$ \\
\hline \multicolumn{2}{|l|}{ C-Ç } \\
\hline Can A & O-67 \\
\hline Canbay A & P-19 \\
\hline Canbay Durmaz S & P-19 \\
\hline Candan B & $\mathrm{O}-40$ \\
\hline Candır BN & $\mathrm{P}-16$ \\
\hline Canlı A & P-09 \\
\hline Cebeci H & P-29 \\
\hline Cezairoğlu MA & $\mathrm{O}-32$ \\
\hline Cirık H & O-77 \\
\hline Coşkun İ & O-59 \\
\hline Coşkun O & $\mathrm{O}-37$ \\
\hline Coşkun Ö & P-01, P-25 \\
\hline
\end{tabular}




\begin{tabular}{|c|c|c|c|}
\hline Cömert A & O-39 & Ertan E & O-58 \\
\hline Cüneyit İ & $\mathrm{O}-02$ & Etli $M$ & O-52 \\
\hline Çalgüner E & $\mathrm{O}-44$ & & \\
\hline Çandır BN & $\mathrm{O}-04, \mathrm{P}-25$ & $\mathbf{F}$ & \\
\hline Çavuş F & $\mathrm{O}-21$ & Fazlığulları Z & $\mathrm{O}-05, \mathrm{O}-07, \mathrm{O}-12, \mathrm{O}-13, \mathrm{O}-14, \mathrm{O}-27$, \\
\hline Çelik G & $\mathrm{O}-53$ & & $\mathrm{O}-43, \mathrm{O}-53, \mathrm{O}-67, \mathrm{O}-72, \mathrm{P}-29$ \\
\hline Çelik H & O-85 & Fidan N & $\mathrm{P}-35$ \\
\hline Çelik S & PL-4 & Firat A & $\mathrm{O}-15$ \\
\hline Çelik Z & $\mathrm{O}-63$ & & \\
\hline Çetin A & $\mathrm{O}-29, \mathrm{P}-35$ & G & \\
\hline Çetin AZ & $\mathrm{O}-35$ & Gayretli Ö & $\mathrm{O}-04, \mathrm{O}-37$ \\
\hline Çetin R & $\mathrm{O}-33$ & Girgin $\mathrm{T}$ & $\mathrm{P}-11$ \\
\hline Çetinok H & O-39 & Gökşan AS & $\mathrm{P}-14, \mathrm{P}-20$ \\
\hline Çınaroğlu S & $\mathrm{O}-47$ & Gözil R & $\mathrm{O}-06, \mathrm{O}-09, \mathrm{O}-10, \mathrm{P}-03$ \\
\hline Çiçekcibaşı AE & $\mathrm{P}-14, \mathrm{P}-21, \mathrm{P}-22, \mathrm{P}-23$ & Gülcen B & $\mathrm{P}-13$ \\
\hline Çiftçi R & O-29 & Güler K & $\mathrm{I}-1$ \\
\hline Çimen K & $\mathrm{O}-50$ & Güler M & O-59 \\
\hline Çimen $M$ & $\mathrm{O}-50$ & Gülhan R & $\mathrm{O}-86$ \\
\hline \multirow[t]{2}{*}{ Çolak İ } & $\mathrm{O}-73$ & Gümeler E & $\mathrm{O}-36$ \\
\hline & & Günenç Beşer C & O-93 \\
\hline D & & Güner Sak N & $\mathrm{P}-26$ \\
\hline Demir İ & $\mathrm{O}-28$ & Güneş Erçakmak B & O-90 \\
\hline Demiryürek D & O-36, O-92, O-93 & Güngör Y & O-11 \\
\hline Denirtaş G & $\mathrm{O}-21$ & Gürlek Çelik N & P-29 \\
\hline Derinöz E & $\mathrm{P}-12$ & Gürses İA & $\mathrm{O}-17, \mathrm{O}-19, \mathrm{O}-37, \mathrm{P}-01, \mathrm{P}-25$ \\
\hline Digilli B & $\mathrm{P}-14, \mathrm{P}-20$ & Gürsoy S & O-11 \\
\hline Dikici R & $\mathrm{O}-40, \mathrm{O}-52$ & Güzelad Ö & O-87 \\
\hline Dikmetas $\mathrm{O}$ & $\mathrm{O}-15$ & & \\
\hline Dinç $M$ & $\mathrm{P}-02$ & $\mathbf{H}$ & \\
\hline Doğan DE & $\mathrm{O}-85$ & Huyut MA & $\mathrm{O}-22$ \\
\hline Doğan ME & $\mathrm{O}-05$ & & \\
\hline Doğancı ÖI & PL-14, O71 & I-İ & \\
\hline Doğanyiğgit Z & O-78 & Ilgaz HB & $\mathrm{O}-63, \mathrm{O}-74$ \\
\hline Doğruyol G & $\mathrm{O}-50$ & Ipor GT & $\mathrm{P}-10$ \\
\hline Dönmez BO & $\mathrm{P}-10$ & Iş1klar S & $\mathrm{O}-28$ \\
\hline Downie SA & O-39 & İlgi S & $\mathrm{P}-04, \mathrm{P}-05$ \\
\hline Durgun B & $\mathrm{I}-5$ & İlgin F & $\mathrm{P}-21$ \\
\hline Durmaz MS & $\mathrm{O}-43$ & İlhan ÇF & $\mathrm{O}-75, \mathrm{O}-84$ \\
\hline Dursun A & $\mathrm{O}-26, \mathrm{O}-46, \mathrm{O}-48, \mathrm{O}-49$ & İnce $F$ & $\mathrm{O}-46$ \\
\hline Durum Polat Y & $\mathrm{O}-20$ & İnce MS & $\mathrm{O}-06, \mathrm{O}-09, \mathrm{O}-10, \mathrm{P}-03$ \\
\hline \multirow[t]{2}{*}{ Durur Subaşı I } & $\mathrm{P}-34$ & İpek ED & O-91 \\
\hline & & İsmailoğlu P & O-61 \\
\hline \multicolumn{4}{|l|}{$\mathbf{E}$} \\
\hline Ekici H & $\mathrm{O}-13$ & $\mathbf{K}$ & \\
\hline Elvan Ö & O-89 & Kabakcı ADA & O-01 \\
\hline Emekli E & $\mathrm{O}-38$ & Kachlík D & $\mathrm{I}-3$ \\
\hline Emirzeoğlu M & $\mathrm{O}-81, \mathrm{P}-32$ & Kadiyoran C & $\mathrm{O}-24, \mathrm{P}-28, \mathrm{P}-30, \mathrm{P}-36$ \\
\hline Er Ulubaba H & $\mathrm{O}-21$ & Kafa İM & $\mathrm{O}-31, \mathrm{O}-56$ \\
\hline Erçakmak Güneş B & $\mathrm{O}-36$ & Kahiloğulları G & PL-8 \\
\hline Erdem H & $\mathrm{O}-55, \mathrm{O}-57, \mathrm{O}-82$ & Kahraman F & $\mathrm{O}-44$ \\
\hline Erdoğan A & $\mathrm{O}-64$ & Kahraman Yıldırım FG & O-61 \\
\hline Ermez MN & $\mathrm{O}-03, \mathrm{P}-18$ & Kalaycıoğlu A & O-94 \\
\hline
\end{tabular}




\begin{tabular}{|c|c|c|c|}
\hline Kanatlı U & $\mathrm{O}-06, \mathrm{O}-09, \mathrm{O}-10, \mathrm{P}-03$ & $\mathbf{M}$ & \\
\hline Kapakin S & $\mathrm{P}-34$ & Macunluoğlu AC & P-16 \\
\hline Kapkaç HA & $\mathrm{O}-75, \mathrm{O}-84$ & Makay Ö & PL-1 \\
\hline Kaptan AY & $\mathrm{O}-06, \mathrm{O}-10, \mathrm{P}-03$ & Maral F & $\mathrm{P}-02, \mathrm{P}-24$ \\
\hline Kara CO & $\mathrm{O}-02, \mathrm{O}-35$ & Mikami B & PL-13 \\
\hline Karaali K & O-64 & Muhtaroğlu M & P-05 \\
\hline Karabulut AK & $\begin{array}{l}\text { O- } 07, \text { O-12, O- } 13, \mathrm{O}-14, \mathrm{O}-27, \mathrm{O}-43 \\
\mathrm{O}-67, \mathrm{O}-72, \mathrm{P}-29\end{array}$ & & \\
\hline Karaca Bozdağ Z & $\mathrm{O}-04, \mathrm{P}-16$ & $\mathbf{N}$ & \\
\hline Karaca Ö & $\mathrm{O}-33$ & Nakamatsu NA & PL-13 \\
\hline Karaçoban L & O-92 & Nteli Chatzioglou G & $\mathrm{O}-37$ \\
\hline Karahan ST & $\mathrm{O}-83$ & & \\
\hline Karakaş Tanır AB & O-51 & O-Ö & \\
\hline Karataş T & $\mathrm{O}-21$ & Oflamaz A & O-78 \\
\hline Karip B & P-06 & Oğuz Ö & $\mathrm{O}-55, \mathrm{O}-57$ \\
\hline Kartal A & $\mathrm{O}-14$ & Ok F & $\mathrm{O}-17$ \\
\hline Kastamoni Y & $\mathrm{O}-26, \mathrm{O}-69, \mathrm{O}-80$ & Onur Ş & $\mathrm{P}-37$ \\
\hline Kaya B & PL-13 & Orhan $M$ & O-66 \\
\hline Kaya B & $\mathrm{P}-22$ & Ortadeveci A & $\mathrm{O}-03, \mathrm{O}-18, \mathrm{P}-17, \mathrm{P}-18$ \\
\hline Keleş A & P-36 & Otağ İ & O-50 \\
\hline Kendir S & $\mathrm{O}-83$ & Öğüt E & $\mathrm{O}-25, \mathrm{O}-87$ \\
\hline Kervancioğlu P & O-66 & Öner Ç & $\mathrm{P}-06$ \\
\hline Kesimal U & P-07 & Öner S & O-60 \\
\hline Keskin $M$ & $\mathrm{P}-24$ & Öner Z & O-60 \\
\hline Keskin N & $\mathrm{O}-65$ & Öner Z & O-70 \\
\hline Kılıç ŞN & $\mathrm{O}-57$ & Öz N & O-64 \\
\hline Kıran H & $\mathrm{P}-17$ & Öz S & $\mathrm{O}-03, \mathrm{P}-18$ \\
\hline Kirazlı Ö & $\mathrm{O}-86$ & Özbağ D & O-21, O-29, O-42, O-76, O-77, P-19 \\
\hline Kısaoğlu A & O-77 & Özcan BN & $\mathrm{O}-81, \mathrm{P}-32$ \\
\hline Kızılay Ö & $\mathrm{P}-27$ & Özcan E & $\mathrm{O}-33, \mathrm{O}-34, \mathrm{P}-11$ \\
\hline Kızılay ÖC & O-91 & Özdemir B & $\mathrm{O}-48, \mathrm{O}-80$ \\
\hline Koca R & $\mathrm{O}-43$ & Özdemir B & O-76 \\
\hline Kocabeyoğlu S & $\mathrm{O}-15$ & Özdemir S & $\mathrm{O}-28$ \\
\hline Kökce A & $\mathrm{P}-33$ & Özden H & $\mathrm{O}-03, \mathrm{O}-18, \mathrm{P} 17, \mathrm{P}-18$ \\
\hline Koplay M & $\mathrm{O}-53, \mathrm{P}-08, \mathrm{P}-14, \mathrm{P}-23, \mathrm{P}-38$ & Özer CM & $\mathrm{O}-45, \mathrm{O}-65$ \\
\hline Korez KM & O-72 & Özkan A & O-87 \\
\hline Korkmaz A & O-79 & Özkan M & $\mathrm{O}-30$ \\
\hline Korkmaz AC & $\mathrm{O}-11$ & Özkan MH & $\mathrm{I}-2$ \\
\hline $\begin{array}{l}\text { Korkmaz C } \\
\text { Köse F }\end{array}$ & $\begin{array}{l}\mathrm{O}-23 \\
\mathrm{O}-77\end{array}$ & Özpar R & $\mathrm{O}-28$ \\
\hline $\begin{array}{l}\text { Kose E } \\
\text { Küçük E }\end{array}$ & $\mathrm{O}-47$ & Özsoy T & O-70 \\
\hline Kürkcüoğlu A & $\mathrm{P}-16$ & Öztürk A & $\mathrm{O}-37$ \\
\hline Kurt C & $\mathrm{P}-13$ & Öztürk K & $\mathrm{O}-26, \mathrm{O}-62, \mathrm{O}-80$ \\
\hline Kurtoğlu Olgunus Z & $\mathrm{O}-16$ & Öztürk Köse Ö & $\mathrm{O}-32$ \\
\hline Kuş İ & $\mathrm{P}-12$ & Öztürk M & O-72 \\
\hline \multirow{2}{*}{ Kuzu MA } & PL-6 & Öztürk S & $\mathrm{O}-44$ \\
\hline & & Öztürk Y & P-04 \\
\hline \multicolumn{4}{|l|}{$\mathbf{L}$} \\
\hline Lafçı Fahrioğlu S & $\mathrm{O}-54, \mathrm{P}-04, \mathrm{P}-05$ & $\mathbf{P}$ & \\
\hline Lee $\mathrm{U}$ & PL-13 & Parlak H & $\mathrm{O}-87$ \\
\hline Lozanoff BK & PL-13 & Paslı B & O-90 \\
\hline Lozanoff S & PL-13 & Polat Çorumlu E & $\mathrm{O}-75, \mathrm{O}-84$ \\
\hline
\end{tabular}




\section{R}

Rettenmeier C

S-Ş

Sağlam L

Salim H

Salkım B

Salman N

Sarı E

Saygin DA

Saylam H

Seçgin Y

Seher N

Sertçelik M

Sevindik B

Sindel M

Sindel T

Sinen $\mathrm{O}$

Smith A

Solgun S

Solmaz E

Süral S

Süzen LB

Şahin Ö

Şahin Öztürkcan D

Şahin Ş

Şanlı O

Şanlı OC

Şehirli ÜS

Şeker B

Şendemir E

Şener U

Şenol D

Şenol RGT

T

Taşpınar Ç

Taştan ÖA

Tekdemir İ

Tellioğlu Metin A

Temizsoy Korkmaz F

Thompson JD

Tokpınar A

Torigoe T

Toy S

Tunalı S

Tuncel Çini N

Tunçyürek Ö

Turan K
PL-13

P-01

O-44

O-12

O-79

O-37

O-01

O-05

O-60

P-08

O-51

O-05

O-44

O-44

O-87

PL-13

P-19

O-05, O-07

O-02

O-64

P-20, P-21

O-14

O-68

O-48

O-26

O-86

$\mathrm{P}-22$

O-58, P-26

O-20

O-29, O-76

O-55

P-08, P-38

PL-14

PL-5

O-20

P-25

PL-13

O-78

PL-13

O-60

PL-11

O-31, P-26, P-31

O-54

O-60
U-Ü

Uçar C

O-76

Uğurlu BN

Ulubaba $\mathrm{H}$

Ulupinar E

Uslu Aİ

Uyanıkgil Y

Uysal III

Uz A

Uzabaci H

Uzmansel D

Uzun A

Uzuner MB

Ülkir M

Ünver Doğan N

V

Vatansever A

Verimli U

Vural A

Vurgun H

Y

Yalçın ED

Yazan $\mathrm{H}$

Yeniel AÖ

Yeşil Kayabaşı Ç

Yiğit M

Yildiran G

Yildırım FB

Yildiz A

Yildiz H

Yildiz S

Yildiz Yilmaz M

Yilmaz İ

Yilmaz MT

Yilmaz N

Yilmaz NA

Yilmaz PD

Yilmaz S

Yilmaz V

Yilmazer Kayatekin AZ

Yolcular B

Yücedağ $\mathrm{H}$

Yükselen A

O-66

O-49, P15

Panel-1, PL-2

O-16

P-01

O-83

O-87

O-77

O-72

O-76

O-59

P-04

O-01, P-28, P-30, P-36

O-77

P-30

O-24

O-78

O-49, P-15

O-45, O-65

O-44

O-90

O-54

Z

Zeybek A O-30

Zeybek ND O-85

Zor KR O-47 